\title{
Quinn's Law of Fluid Dynamics Pressure-Driven Fluid Flow through Closed Conduits
}

\author{
Hubert M Quinn' ${ }^{1} \dagger$ \\ ${ }^{1}$ Department of Research and Development, The Wrangler Group LLC, 40 Nottinghill Road, Brighton, Ma.02135, USA. \\ Corresponding: hubert@wranglergroup.com
}

\begin{abstract}
In this paper we develop from first principles a unique law pertaining to the flow of fluids through closed conduits. This law, which we call "Quinn's Law", may be described as follows:

When fluids are forced to flow through closed conduits under the driving force of a pressure gradient, there is a linear relationship between the normalized dimensionless pressure gradient, $P_{Q}$, and the normalized dimensionless fluid current, $C_{Q}$. The relationship is expressed mathematically as:

$$
P_{Q}=k_{1}+k_{2} C_{Q}
$$

This linear relationship remains the same whether the conduit is filled with or devoid of solid obstacles. The law differentiates, however, between a packed and an empty conduit by virtue of the tortuosity of the fluid path, which is seamlessly accommodated within the normalization framework of the law itself. When movement of the fluid is very close to being at rest, i.e., very slow, this relationship has the unique minimum constant value of $\mathrm{k}_{1}$, and as the fluid acceleration increases, it varies with a slope of $\mathrm{k}_{2}$ as a function of normalized fluid current.
\end{abstract}

Quinn's Law is validated herein by applying it to the data from published classical studies of measured permeability in both packed and empty conduits, as well as to the data generated by home grown experiments performed in the author's own laboratory.

Keywords: permeability; porous media; fluid dynamics; fluid current; turbulence; simple harmonic motion 
†Email address for correspondence: hubert@wranglergroup.com.

\section{A. The Problem}

The history of attempts to quantify a relationship between fluid flow in closed conduits (whether it be in a packed conduit or in an empty conduit) and the relevant variables governing that relationship dates back at least to the work of Darcy in 1856 [1]. Since that time, a host of models have been proposed with their accompanying array of equations, each of which has its own limitations and restrictions [2]. Accordingly, the scientific literature is replete with reports of experimental results aimed at trying to resolve the many discrepancies which litter the fluid dynamics landscape [3]. In fact, this field of study is in such disarray that there is no currently accepted theory of fluid dynamics from which one could derive an analytical solution to the supposed governing equation of fluid dynamics, the Navier-Stokes equation [4].

This author has devoted his entire career to doing fluid flow measurements, predominantly in packed beds relating to the field of HPLC (High Pressure Liquid Chromatography) [5, 6, 7, 8, 9, $10,11,12$.]. Rather than nibbling at the fringes of this complicated field of study by trying to manipulate one of the currently accepted theories, a completely different approach was developed. The subsequent model, which we refer to as the Quinn Fluid Flow Model (QFFM) took approximately 20 years to formulate, was developed from first principles, and is different from any other model currently extant. Most importantly, it is a universal theory which applies to all fluid flow embodiments, regardless of whether they contain particles and regardless of the regime of flow in which they are operated. Moreover, it has been validated by testing it against a host of generally accepted experimental data reported in the literature, as well as this author's own measurements.

The dimensional manifestation of the QFFM is a complex equation containing many independent and dependent variables and is the properly formatted version of the continuity equation for fluid flow in closed conduits. On the other hand, there is a dimensionless manifestation of the QFFM, which we refer to as Quinn's Law, which is a simple linear relationship between the reduced pressure gradient and the reduced fluid flow current in a closed conduit. In contrast to the complicated alternatives that conventional theories of fluid dynamics have to offer, Quinn's Law is very simple. It has just two variable parameters. More importantly, and in contrast to conventional theories, it is valid over the entire range of the fluid flow regime, providing analytical solutions in laminar, transitional and fully turbulent ranges of fluid flow.

This author's development of the QFFM was born of the frustration experienced in trying to reconcile experimental data and textbook teachings relative to packed/empty conduit permeability. For instance, the chromatographic literature abounds with erroneous teachings: incorrect use of velocity frames [13]; the notion that the particle size is defined by permeability (14); violations of Continuity Laws [15, 16, 17.]; self-serving validations [18]. Likewise, the engineering literature is littered with similar errors: misapplication of porosity function [19]; erroneous derivation of the viscous constant [20]; "blind leading the blind" syndrome [21]; mathematicians using computers to produce mind-numbing computations, which purport to explain equations that have no connection to fluid flow parameters in the first place [22]; contradictory statements of facts [23]; etc.; etc. Moreover, both disciplines are especially guilty 
of neglecting the role of kinetic considerations in favor of focusing too much on the less complex laminar flow regime.

\section{B. The Solution-An Introduction}

In order to underscore the importance of achieving a comprehensive understanding of the physics underlying our theoretical development, rather than just the mathematical framework, we want to introduce upfront the important novel concepts which are part of the development of our solution to the problem. In this way, we hope the reader will be focused more on where these concepts fit into the overall comprehensive understanding, rather than becoming embroiled in the mathematics, which, after all, is simply the gravel of which the roadway is made, and is not a critical component to a discussion of what is the destination.

\section{a. Q- hypothetical particles}

This idea is a very simple one. It is a way of subdividing the free space in an empty conduit, using the same mathematics that we use to assign free space to solid particles in a packed conduit. We accomplish this by extending the existing framework of particle porosity for packed conduits containing solid particles to accommodate particles of free space (no solid skeleton) in an empty conduit. The resultant framework is a universe of two mathematical half-planes, each of which is the mirror image of the other. Of particular importance in this theoretical development, however, is that we manage to avoid the point of discontinuity which would otherwise arise as a consequence of the theory at the axis of symmetry between the two mathematical half-planes, since this would cause the mathematics and, consequently, the entire logic to implode at this location. We accomplish this task by only using the absolute value of the particle fraction [abs $\left.\left(1-\varepsilon_{0}\right)\right]$ function, which is always finite, in our definition of the Hypothetical Q Channel (HQC) diameter, $d_{c}$, which forms the basis of the QFFM. In other words, we only use that portion of the theory which is valid in the real world. In addition, we are careful to manage our mathematical framework to incorporate the Conservation Laws into our HQC by a mechanism which reconciles particle diameter, $\mathrm{d}_{\mathrm{p}}$, and conduit external porosity, $\varepsilon_{0}$.

\section{b. rh-the fluid drag normalization coefficient}

The concept underlying this parameter is, arguably, the most important element of the entire QFFM theory. It is the exchange rate necessary to create a common "currency" between viscous and kinetic considerations dictated by the Laws of Nature. We use the value of the ratio of surface area to cross-sectional area of a spherical particle, $\mathrm{r}_{\mathrm{h}}$, i.e., 4 , to define our control volume, $\left(4 \pi \mathrm{r}_{\mathrm{h}}{ }^{3} / 3=268\right)$, making this value the common denominator which connects the control volume, surface area, cross sectional area and fluid drag. Accordingly, in our permeability model, this common denominator takes on the dual role of (a) being the radius of the obstacle responsible for the fluid friction due to viscous considerations, and, (b) at the same time, represents the hydraulic radius of the flow channel responsible for the fluid friction due to kinetic considerations. We exploit this feature in our theory by providing a mechanism for normalizing fluid resistance by either (1) surface area contact $\left(\mathrm{k}_{1}=4 \pi \mathrm{r}_{\mathrm{h}}{ }^{2} / 3=67\right)$, or, (2) reciprocal channel circumference $\left(\mathrm{k}_{2}=\right.$ $\left.1 /\left(2 \pi r_{h}\right)=1 / 25\right)$. 


\section{c. $\delta$ - the porosity normalization coefficient}

This parameter represents one of the cornerstones of our theory which allows us to accommodate both packed and empty conduits. It is a specific property of the flow embodiment under study. For instance, the same packed conduit with $\mathrm{x}$ number of particles will have a different value for $\delta$, than the same conduit packed with y number of the same particles. Thus, a packed and an empty conduit will also have different $\delta$ values. Our $\delta$ parameter establishes in our theory (and is confirmed by our pressure drop measurements) that the pressure drop in the kinetic term of the permeability equation is inversely proportional to the $6^{\text {th }}$ power of the conduit external porosity. By combining our parameter $\delta$ with the modified Reynolds number, $\mathrm{R}_{\mathrm{em}}$, we create a unique grouping of terms, our $Q_{N}$ parameter, which we term fluid current. This parameter is the engine that drives the fluid velocity profile and, accordingly, reconciles all forms of flow.

\section{d. $\gamma$-the architectural normalization coefficient}

This parameter is a rather obvious development to anyone who has experience in building physical structures from scratch, such as a house or barn or wall, all of which are made of building blocks of wood/bricks/stones and, accordingly, forms the basis for one's thinking when one builds a packed conduit from building blocks of particles (stones) within a fixed volume of free space, i.e. a conduit.

\section{e. $\lambda$-the wall effect normalization coefficient of fluid current}

This parameter is another cornerstone in our framework which aligns the physics of a packed and empty conduit, but only manifests in the kinetic term. It is also a specific property of the flow embodiment under study, and only changes as a function of the specific wall effect of a given packed conduit. In this case, however, the effect of the wall is transferred to the motion of the fluid, which is why we describe it as a component of fluid current. The rationale behind this parameter is fairly well documented in the main body of our paper, wherein we took advantage of Prandtl's concept of the boundary layer in formulating our definition of the primary wall effect [24].

\section{f. $\tau$-the tortuosity normalization coefficient}

We believe that tortuosity is primarily a function of channel architecture normalized for conduit external porosity. Accordingly, we have defined this parameter as another normalization coefficient which, again, only manifests in the kinetic term of the permeability equation. It compensates for the different flow paths which are generated within different packed conduits, as well as compensating for the unique flow path generated within all empty conduits.

\section{g. The Harmonic Oscillator}

Finally, everything comes together in our notion that fluid flow in closed conduits, whether packed with solid particles or empty, is a form of harmonic motion. Intuition tells us that we ought to imagine that the fluid going through a packed bed has to somehow double back upon itself. This idea led to the concept of Simple Harmonic Motion (SHM). Once having understood 
that SHM was the operating principle governing fluid flow in closed conduits, the connection to uniform circular motion became obvious. This led to the realization that when the speed of the fluid at the wall is zero, the velocity is not zero because the fluid is changing direction simultaneously. This was enough to establish the viscous friction factor as the underlying concept behind the harmonic oscillator algorithm. Once having done this, it was relatively easy to figure out what parameter was what, in the fluid motion, and make a one-to-one correspondence between the dimensionless fluid viscous friction factor parameters and the dimensional simple harmonic motion parameters, which, incidentally, is the reason why the particular units of measure in the SHM can be arbitrary, as long as they are self-consistent. More accurately stated, we incorporate the framework of damped-SHM to accommodate the impact of conduit wall friction and fluid internal friction, using both as damping coefficients on the overall motion of the fluid.

The concept of "fluid chaos", which permeates conventional wisdom, is rejected in our theory. The best way to think about it is to imagine oneself as a hunter in the mountains. The deer, which is the focus of our hunt, is perfectly camouflaged against the background of the hillside. Thus, the deer is invisible to the hunter, despite the fact that the deer's body is located in free space in all three dimensions, i.e., it has location coordinates in the $\mathrm{x}, \mathrm{y}$ and $\mathrm{z}$ planes. The reason why the deer is invisible to the hunter is that, relative to the hillside background, the hunter cannot differentiate between the deer and the underbrush. It is only when the deer changes its positional coordinates relative to the hill in the background that the hunter can see it i.e., when the deer starts running. Moreover, because the movement of the deer is not restricted in terms of free space on the mountainside, his "movement profile" can be erratic, uniform in a straight line (if he follows a road, for instance), or combinations of the above, all driven by the whim of the deer's instincts. However, it is never chaotic or unpredictable, in a scientific context, that is.

In our Hypothetical Q Channel model, the fluid is equivalent to the deer, i.e., it has positional coordinates in all three planes, but the fluid flow profile cannot be distinguished from the background of the channel because its positional coordinates are indistinguishable relative to the conduit wall or conduit center line. It is only when we view the fluid through the "scope" of the $Q_{N}$ parameter ( $4^{\text {th }}$ dimension), that its positional coordinates are changed relative to the background conduit wall/center line. In addition, when viewing the fluid profile through the $\mathrm{Q}_{\mathrm{N}}$ scope, the $Q_{N}$ crosshairs must be spaced closely enough together, relative to the size of $\mathrm{T}$, the time constant of the fluid motion, to generate a comprehensive image of the flow profile, just as the crosshairs in the hunter's telescopic sight must be adjusted, commensurate with the overall size of the deer.

Additionally, and in contrast to the movement of the deer, the fluid movement in our HQC is restricted in free space because of its driving force, i.e., the pressure drop $(\Delta \mathrm{P})$. Furthermore, when we normalize the pressure drop for the length of the conduit, L, i.e., the pressure gradient $(\Delta \mathrm{P} / \mathrm{L})$, we restrict the movement of the fluid even further, i.e., to just the cross-section of the HQC. Thus, as the fluid moves over the cross-section, its current is influenced by the walls of the conduit, i.e., wall friction. Because of internal fluid friction, as its speed increases, the fluid flow profile loses its "arc of motion" and, ultimately, simply moves back and forth over the crosssection in a virtual straight line, i.e., "plug flow". Thus, cross-channel mixing is greatly enhanced by convection which is driven by the kinetic term in the permeability equation. 
The Hypothetical Q Channel, of course, does not exist in the real world. However, the model allows us to imagine the fluid moving through the conduit and when we do, what we see is not disorganized or chaotic motion, but a structural pattern characterized by SHM, regardless of Reynolds number or any conduit or particle parameter. Accordingly, we suggest that "fluid chaos" is in the eye/mind of the beholder.

\section{h. Conventional Permeability Equations}

Finally, having started at the beginning of our development with fundamental definitions, we make our way systematically through the governing equations, in order to arrive at the equation most useful to the practitioner of permeability. In so doing, we are mindful to connect our QFFM to the conventional Poiseuille and Ergun flow models, albeit through a modified version for terms, which corrects for the glaring shortcomings of each.

\section{Fundamentals of the Q Fluid Flow Model (QFFM)}

\section{a. Particle}

1.10 Let us define an obstacle to be placed within a packed conduit as a spheroidal particle of nominal diameter $\mathrm{d}_{\mathrm{pm}}$ and sphericity $\Omega_{\mathrm{p}}$. Then we may write:

$$
\mathrm{d}_{\mathrm{p}}=\mathrm{d}_{\mathrm{pm}} \Omega_{\mathrm{p}}
$$

Where, $\mathrm{d}_{\mathrm{p}}=$ the spherical particle diameter equivalent $\Omega_{\mathrm{p}} \leq 1$; thus, when $\Omega_{\mathrm{p}}=1$, the particle is spherical.

Let the particle have a specific pore volume of $S_{\mathrm{pv}}$, a skeletal density of $\rho_{\mathrm{sk}}$, and a mass of $\mathrm{m}_{\mathrm{p}}$.

1.11 Let us define other particle characteristics as:

$$
\mathrm{SA}_{\mathrm{p}}=\pi \mathrm{d}_{\mathrm{p}}^{2}
$$

And

$$
\mathrm{CSA}_{\mathrm{p}}=\frac{\pi \mathrm{d}_{\mathrm{p}}^{2}}{4}
$$

Where, $\mathrm{SA}_{\mathrm{p}}=$ particle equivalent surface area; and $\mathrm{CSA}_{\mathrm{p}}=$ particle equivalent cross sectional area. 
It follows that we may write:

$$
\begin{aligned}
& \mathrm{V}_{\mathrm{dp}}=\frac{\pi \mathrm{dp}_{\mathrm{p}}^{3}}{6} \\
& \rho_{\text {part }}=\frac{\mathrm{m}_{\mathrm{p}}}{\mathrm{V}_{\mathrm{dp}}}
\end{aligned}
$$

Where, $\mathrm{V}_{\mathrm{dp}}=$ the volume of a single spherical particle equivalent; $\rho_{\text {part }}=$ the apparent particle density.

1.12 Let us define particle porosity as the ratio of free space within the particle to the total free space occupied by the particle as a whole, thus:

$$
\varepsilon_{\mathrm{p}}=\mathrm{S}_{\mathrm{pv}} \rho_{\mathrm{part}}
$$

Where, $\varepsilon_{\mathrm{p}}=$ the particle porosity.

It follows that:

When, $\varepsilon_{\mathrm{p}}=1$, the particle is devoid of solid matter, i.e. contains only free space;

When, $\varepsilon_{\mathrm{p}}=0$, the particle is made entirely of solid matter, i.e. the particle is non-porous;

When, $0 \leq \varepsilon_{\mathrm{p}}<1$, the particle is partially porous, i.e., consists of a solid particle skeleton plus internal pores.

\section{b. Conduit}

1.13 Let us define a fluid conduit as a right circular cylinder of length $L$ and diameter $D$. Then we may write:

$$
\mathrm{V}_{\mathrm{ec}}=\frac{\pi \mathrm{D}^{2} \mathrm{~L}}{4}
$$

Where, $\mathrm{V}_{\mathrm{ec}}=$ the volume of free space within an empty conduit.

Let the conduit be packed with $n_{p}$ number of particle equivalents of diameter $d_{p}$. 
It follows that we may write:

$$
\mathrm{V}_{\mathrm{part}}=\frac{\underline{\mathrm{n}}_{\mathrm{p}} \frac{\pi \mathrm{d}_{\mathrm{p}}^{3}}{6}}{6}
$$

Where, $\mathrm{V}_{\text {part }}=$ the cumulative volume occupied by all the particle equivalents within a packed conduit.

1.14 Let us define as $n_{p q}$, the number of particle equivalents whose collective volume is equal to the volume of free space within an empty conduit.

It follows that we may write:

$$
\mathrm{n}_{\mathrm{pq}}=\frac{\mathrm{V}_{\mathrm{ec}}}{\mathrm{V}_{\mathrm{dp}}}=\frac{3 \mathrm{D}^{2} \mathrm{~L}}{2 \mathrm{~d}_{\mathrm{p}}^{3}}
$$

1.15 Let us define the packed conduit fluidic architecture as:

$$
\gamma=\underline{\mathrm{n}} \underline{\mathrm{n}} \underline{\mathrm{D}}
$$

Where, $\gamma=$ the fluidic architectural coefficient for a given packed conduit.

We now turn to conduit porosities.

1.16 Let us define the volume of free space within the packed conduit which is external to all the particles as $\mathrm{V}_{\mathrm{e}}$; the volume of free space which is internal to all the particles as $\mathrm{V}_{\mathrm{i}}$; the volume which is occupied by all the particle skeletons as $\mathrm{V}_{\mathrm{sk}}$; and $\mathrm{V}_{\mathrm{t}}$ as the total volume of free space within the packed conduit which is devoid of solid matter.

It follows that we may write:

$$
\left(1-\varepsilon_{0}\right)=\frac{\mathrm{n}_{\mathrm{p}}}{\mathrm{n}_{\mathrm{pq}}}=\frac{\mathrm{V}_{\mathrm{part}}}{\mathrm{V}_{\mathrm{ec}}}
$$

Where, the conduit particle fraction, $\left(1-\varepsilon_{0}\right)=$ the volume fraction of the packed conduit occupied by the particles.

$$
\varepsilon_{\mathrm{sk}}=\frac{\left(1-\varepsilon_{\mathrm{p}}\right) \mathrm{n}_{\mathrm{p}}-}{\mathrm{n}_{\mathrm{pq}}}=\frac{\mathrm{V}_{\mathrm{sk}}}{\mathrm{V}_{\mathrm{ec}}}
$$


Where, the conduit skeletal porosity, $\varepsilon_{\mathrm{sk}}=$ the volume fraction of the packed conduit occupied by the particle skeletons.

$$
\varepsilon_{0}=1-\underline{\mathrm{n}}_{\mathrm{p}}=\frac{\mathrm{V}_{\mathrm{e}}}{\mathrm{n}_{\mathrm{pq}}}
$$

Where, the conduit external porosity, $\varepsilon_{0}=$ the volume fraction of the packed conduit external to the particles.

$$
\varepsilon_{\mathrm{i}}=\varepsilon_{\mathrm{p}}\left(1-\varepsilon_{0}\right)=\frac{\mathrm{V}_{\mathrm{i}}}{\mathrm{V}_{\mathrm{ec}}}
$$

Where, the conduit internal porosity, $\varepsilon_{\mathrm{i}}=$ the volume fraction of the packed conduit internal to the particles.

$$
\varepsilon_{\mathrm{t}}=1-\frac{\left(1-\varepsilon_{\mathrm{p}}\right) \underline{n}_{\mathrm{p}}}{\mathrm{n}_{\mathrm{pq}}}=\varepsilon_{\mathrm{i}}+\varepsilon_{0}
$$

Where, the conduit total porosity, $\varepsilon_{\mathrm{t}}=$ the sum of the volume fractions external and internal to the particles.

It follows that particle porosity and conduit internal porosity are related as follows:

when $\varepsilon_{\mathrm{p}}=0$, conduit internal porosity $\varepsilon_{\mathrm{i}}=0$ and, thus, the particles are completely solid throughout, i.e., non-porous.

when $\varepsilon_{\mathrm{p}}=1$, conduit internal porosity $\varepsilon_{\mathrm{i}}=\left(1-\varepsilon_{0}\right)$ and thus, the particles are completely devoid of solid matter, i.e., totally porous.

Additionally, it follows that reconciling the definitions above for solid matter and lack thereof, i.e., porosity, within a conduit, [see Eqs. (8) and (11) above], we may now write:

$$
\underline{\mathrm{n}}_{\mathrm{p}} \frac{\pi \mathrm{d}_{\mathrm{p}}^{3}}{6}=\mathrm{V}_{\mathrm{ec}} \mathrm{abs}\left(1-\varepsilon_{0}\right)
$$

Equation (16) reconciles the distribution of free space within the conduit according to the conservation Laws of Nature, whereby all partial volume fractions of the fluid-filled packed conduit, whether occupied by solid matter or fluid, add to unity. 


\section{c. The Conservation Laws governing packed conduits}

1.17 Thus, the Conservation Laws pertaining to packed conduits dictate that we may write:

$$
\begin{gathered}
\varepsilon_{0}+\varepsilon_{\mathrm{i}}+\varepsilon_{\mathrm{sk}}=1 \\
\text { or } \\
\varepsilon_{\mathrm{t}}+\varepsilon_{\mathrm{sk}}=1
\end{gathered}
$$

1.18 Let us define the packing density of a packed conduit as:

$$
\rho_{\text {pack }}=\frac{\mathrm{M}_{\mathrm{p}}}{\mathrm{V}_{\mathrm{ec}}}
$$

Where, $\rho_{\text {pack }}=$ the packing density of the packed conduit; $M_{p}=n_{p} m_{p}$, the total mass of all the particles in a packed conduit under study.

It follows that we may now write:

$$
\begin{gathered}
\varepsilon_{0}=1-\rho_{\mathrm{pack}}\left(\mathrm{S}_{\mathrm{pv}}-1 / \rho_{\mathrm{sk}}\right) \\
\text { or } \\
\varepsilon_{0}=1-\left[2 \mathrm{n}_{\mathrm{p}} \mathrm{d}_{\mathrm{p}}^{3} /\left(3 \mathrm{D}^{2} \mathrm{~L}\right)\right]
\end{gathered}
$$

Accordingly, we may write:

$$
\varepsilon_{\mathrm{p}}=\frac{\left(\varepsilon_{\mathrm{t}}-\varepsilon_{0}\right)}{\left(1-\varepsilon_{0}\right)}
$$

Substituting for the independently measured components of $\varepsilon_{\mathrm{p}}$ in Equation (22), gives

$$
S_{\mathrm{pv}} \rho_{\mathrm{part}}=\frac{\left(\varepsilon_{\mathrm{t}}-\underline{\varepsilon}_{0}\right)}{\left(1-\varepsilon_{0}\right)}
$$

It therefore follows that, empirically, we may define a packed conduit in terms of 4 independent variables $\left(\mathrm{M}_{\mathrm{p}}, \mathrm{V}_{\mathrm{ec}}, \mathrm{S}_{\mathrm{pv}}, \rho_{\mathrm{sk}}\right)$ or, alternatively, $\left(\mathrm{n}_{\mathrm{p}}, \mathrm{d}_{\mathrm{p}}, \mathrm{D}, \mathrm{L}\right)$, in combination with one dependent 
variable $\left(\varepsilon_{0}\right)$, all of which are measureable. However, if in addition to measuring the independent variables, one also measures the value of the external porosity, $\varepsilon_{0}$ (a dependent variable), both sides of Equations (22) and (23) must be reconciled for any given packed conduit under study, as dictated by the Conservation Laws (sometimes referred to as the Laws of Continuity when their application involves moving entities like the fluid in this particular application). This dictate from the Laws of Continuity trumps all measurement techniques, which generally lack the specificity/accuracy to balance either equation without the need for further reconciliation or modification.

Thus, the left hand side of Equation (23) contains measurements made outside of the packed conduit, i.e., independent of the packed conduit under study, whereas the right hand side of Equation (23) contains measurements made within the packed conduit under study. Accordingly, balancing of Equation (23) is always necessary to validate the accuracy of the reported values for the measured parameters of the packed conduit under study.

It follows that, in the case of packed conduits which contain nonporous particles, Equation (23) is equal to zero on both sides of the equalization sign, thus eliminating the need to reconcile column porosity and particle porosity.

\section{d. The Q-Porosity Function ( $\varepsilon$ )}

1.19 Let us now collect all the partial porosity definitions in the QFFM underlying packed conduits which are defined in terms of particle size equivalents and view them as dimensionless mathematical functions of $n_{p}$, which we will designate as Q-Porosity functions. There are a total of 5 such functions, which we view in the context of the generalized Q-Porosity function $\varepsilon$.

1. $\left(1-\varepsilon_{0}\right)=\mathrm{n}_{\mathrm{p}} / \mathrm{n}_{\mathrm{pq}}$, Equation (11) above

2. $\varepsilon_{\mathrm{sk}}=\left(1-\varepsilon_{\mathrm{p}}\right) \mathrm{n}_{\mathrm{p}} / \mathrm{n}_{\mathrm{pq}}$, Equation (12) above

3. $\varepsilon_{0}=\left(1-\mathrm{n}_{\mathrm{p}} / \mathrm{n}_{\mathrm{pq}}\right)$, Equation (13) above

4. $\varepsilon_{\mathrm{i}}=\varepsilon_{\mathrm{p}}\left(\mathrm{n}_{\mathrm{p}} / \mathrm{n}_{\mathrm{pq}}\right)$, Equation (14) above

5. $\varepsilon_{\mathrm{t}}=1-\left(1-\varepsilon_{\mathrm{p}}\right) \mathrm{n}_{\mathrm{p}} / \mathrm{n}_{\mathrm{pq}}$, Equation (15) above

It now becomes obvious that the Q-Porosity functions $\varepsilon_{0}$ and $\left(1-\varepsilon_{0}\right)$ are independent of the value of the particle porosity, $\varepsilon_{\mathrm{p}}$.

Similarly, it is also obvious that the Q-Porosity functions $\varepsilon_{\mathrm{i}}, \varepsilon_{\mathrm{sk}}$ and $\varepsilon_{\mathrm{t}}$ are dependent on the value of the particle porosity, $\varepsilon_{\mathrm{p}}$. 


\section{The Conduit Packing Process}

\section{a. Solid Particles $\left(0 \leq \varepsilon_{p}<1\right)$}

1.10 Let us now define the conduit packing process in the case of solid particles $\left(0 \leq \varepsilon_{\mathrm{p}}<1\right)$ by viewing the role of our independent variable, $\mathrm{n}_{\mathrm{p}}$, within the context of the Q-Porosity function $(\varepsilon)$. This is best accomplished by viewing a worked example on a plot of the dimensionless QPorosity function, $\varepsilon$, versus the number of particle equivalents, $\mathrm{n}_{\mathrm{p}}$.

Our chosen worked example consists of 10 micron particles packed into a conduit of dimensions $10 \mathrm{~cm}$ in length and $0.46 \mathrm{~cm}$ in diameter, the details for which are captured as a spreadsheet in table 1 , for the case in which the particles are nonporous $\left(\varepsilon_{\mathrm{p}}=0\right)$.

Table 1. The Q-Porosity Function $(\varepsilon)$ for nonporous particles $\left(\varepsilon_{\mathrm{p}}=0\right)$

\begin{tabular}{|c|c|c|c|c|c|c|c|c|c|c|c|c|c|c|c|c|}
\hline Particle & $\mathrm{L}$ & D & $\mathbf{V}_{\mathrm{ec}}$ & $d_{p}$ & $\mathbf{V}_{\mathrm{dp}}$ & $\mathbf{n}_{\mathrm{pq}}$ & $\gamma$ & $\mathbf{n}_{\mathrm{p}}$ & $\varepsilon_{p}$ & $\left(1-\varepsilon_{0}\right)$ & $\operatorname{abs}\left(1-\varepsilon_{0}\right)$ & $\varepsilon_{0}$ & $\varepsilon_{\mathrm{i}}$ & $\varepsilon_{\mathrm{t}}$ & $\varepsilon_{\text {sk }}$ & Cont. \\
\hline Type & & & & & & & & & & & & & & & & $\left(\varepsilon_{\mathrm{t}}+\varepsilon_{\mathrm{sl}}\right)$ \\
\hline N/A & $\mathrm{cm}$ & $\mathrm{cm}$ & $\mathrm{cm}^{3}$ & $\mathrm{~cm}$ & $\mathrm{~cm}^{3}$ & none & none & none & none & none & none & none & none & none & none & none \\
\hline Solid & 10 & 0.46 & 1.66 & 0.001 & 5.E-10 & $3 . E+09$ & $2 . E+08$ & $3.33 \mathrm{E}+09$ & 0.00 & 1.00 & 1.00 & 0.00 & 0.00 & 0.00 & 1.00 & 1.00 \\
\hline \multirow[t]{7}{*}{ Particles } & 10 & 0.46 & 1.66 & 0.001 & 5.E-10 & 3. $E+09$ & $2 . E+08$ & $2.46 \mathrm{E}+09$ & 0.00 & 0.74 & 0.74 & 0.26 & 0.00 & 0.26 & 0.74 & 1.00 \\
\hline & 10 & 0.46 & 1.66 & 0.001 & 5.E-10 & $3 . E+09$ & $2 . E+08$ & $2.06 \mathrm{E}+09$ & 0.00 & 0.62 & 0.62 & 0.38 & 0.00 & 0.38 & 0.62 & 1.00 \\
\hline & 10 & 0.46 & 1.66 & 0.001 & 5.E-10 & 3.E+09 & $2 . E+08$ & $2.00 \mathrm{E}+09$ & 0.00 & 0.60 & 0.60 & 0.40 & 0.00 & 0.40 & 0.60 & 1.00 \\
\hline & 10 & 0.46 & 1.66 & 0.001 & 5.E-10 & $3 . E+09$ & $2 . E+08$ & $1.93 \mathrm{E}+09$ & 0.00 & 0.58 & 0.58 & 0.42 & 0.00 & 0.42 & 0.58 & 1.00 \\
\hline & 10 & 0.46 & 1.66 & 0.001 & $5 . \mathrm{E}-10$ & $3 . E+09$ & $2 . E+08$ & $1.83 \mathrm{E}+09$ & 0.00 & 0.55 & 0.55 & 0.45 & 0.00 & 0.45 & 0.55 & 1.00 \\
\hline & 10 & 0.46 & 1.66 & 0.001 & 5.E-10 & 3. $E+09$ & $2 . E+08$ & $8.65 E+08$ & 0.00 & 0.26 & 0.26 & 0.74 & 0.00 & 0.74 & 0.26 & 1.00 \\
\hline & 10 & 0.46 & 1.66 & 0.001 & 5.E-10 & 3. $E+09$ & 2. $E+08$ & $2.00 \mathrm{E}+08$ & 0.00 & 0.06 & 0.06 & 0.94 & 0.00 & 0.94 & 0.06 & 1.00 \\
\hline
\end{tabular}

As shown in table 1, our empirical packing process recognizes Kepler's conjecture regarding the stacking of solid spheres. Accordingly, in practice and as shown in fig.1, the maximum value of the Q-Porosity function $\left(1-\varepsilon_{0}\right)$ is approximately 0.74 with the corresponding minimum value of approximately 0.26 for the Q-Porosity function $\varepsilon_{0}$. Kepler's conjecture is a consequence of the fact that solid spheres and free space are mutually exclusive and therefore the maximum value of $\mathrm{n}_{\mathrm{p}}$ achieved empirically must be less than the value of $\mathrm{n}_{\mathrm{pq}}$. The upper limit of the value of $\mathrm{n}_{\mathrm{p}}$ is always $n_{p q}$ since it represents the most particle equivalents, theoretically, that could be packed into any given conduit under study. 
Accordingly, the theoretical domain of the Q-Porosity function $(\varepsilon)$ runs from 0 to $\mathrm{n}_{\mathrm{pq}}$ and the ranges of the function vary between the values of 0 and 1 , as shown in fig. 1 .

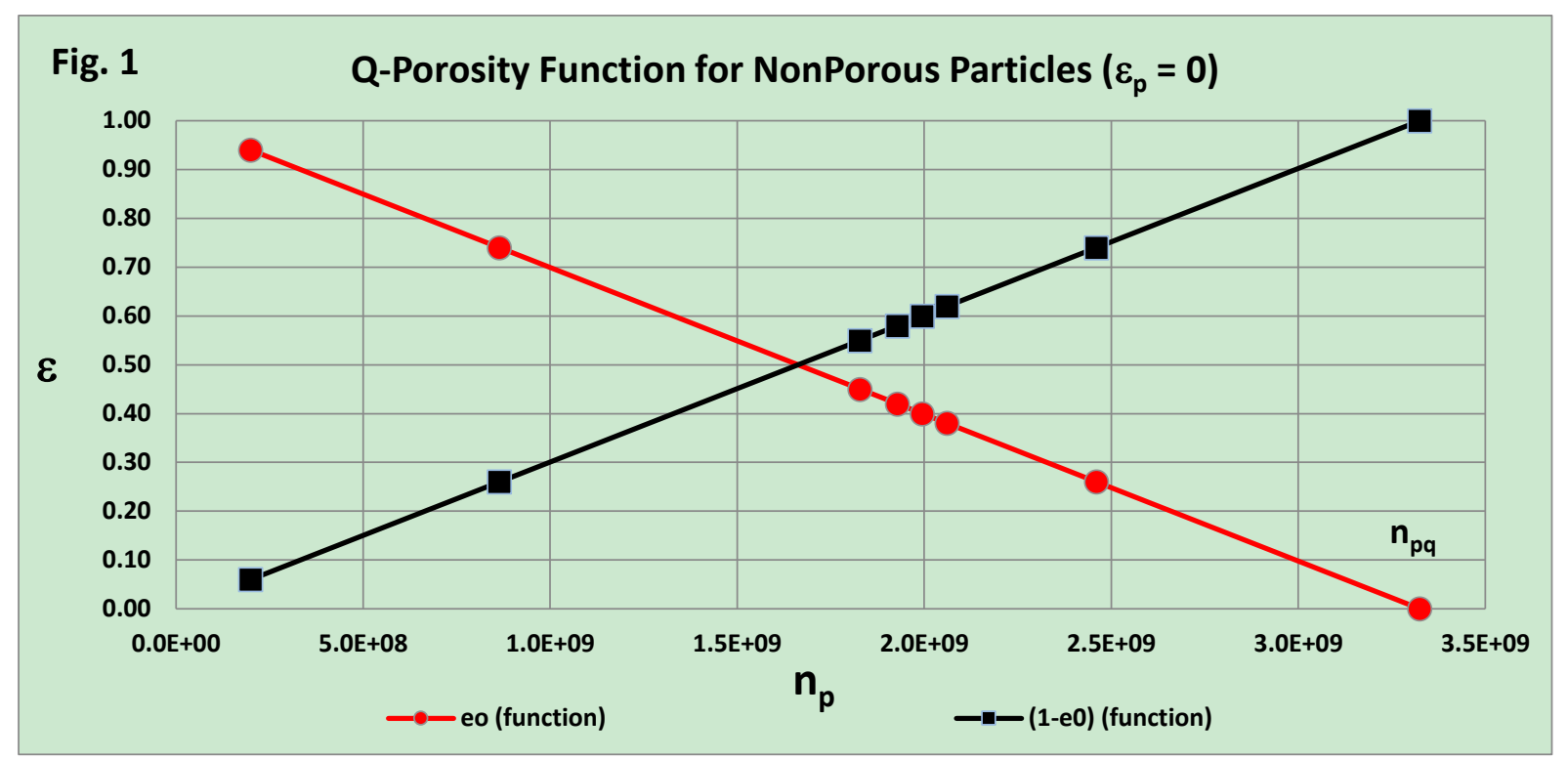

In fig. 1 we only display two of the Q-Porosity functions, i.e., $\varepsilon_{0}$ and $\left(1-\varepsilon_{0}\right)$, since they are not influenced by the particle porosity value, $\varepsilon_{\mathrm{p}}$, and, in this particular case $\left(\varepsilon_{\mathrm{p}}=0\right), \varepsilon_{\mathrm{i}}=0, \varepsilon_{\mathrm{t}}=\varepsilon_{0}$ and $\varepsilon_{\mathrm{sk}}=\left(1-\varepsilon_{0}\right)$. Additionally, we note that these two functions are reciprocal in nature to the extent that as one increases, the other decreases, all as a function of $n_{p}$.

We now further refine the definition of the entity $\mathbf{n}_{\mathbf{p}}$ to be that of a vector rather than a scalar quantity and, therefore, confer upon it a directional component in addition to its mandatory magnitude component.

Let us now define the packing process of a conduit, using our example with solid particles, in terms of our mathematical Q-Porosity functions, as the direction of increasing positive values of $\mathrm{n}_{\mathrm{p}}$. Thus, as we move along the $\mathrm{x}$ axis of fig. 1 in the direction of left to right, starting at the origin of the plot at $\mathbf{n}_{\mathbf{p}}=0$, the corresponding values on the $\mathrm{y}$ axis represent the changing characteristics of the Q-Porosity function $\varepsilon$ in the filling (packing) process. At the starting point of $\mathbf{n}_{\mathbf{p}}=0$, the conduit is devoid of particles (contains only free space) and at the maximum value of $\mathbf{n}_{\mathbf{p}}$ achieved in the filling process, the conduit is fully packed. Accordingly, filling of a "packed" conduit with solid particles is represented by the increasing positive values of $\mathbf{n}_{\mathbf{p}}$, i.e., the motion left to right along the $\mathrm{x}$ axis of the plot starting at the value of $\mathbf{n}_{\mathbf{p}}=0 \rightarrow$.

We shall now consider the more complex packing process in which the particle porosity varies between the values of 0 and 1, i.e., the case of partially porous particles $\left(0<\varepsilon_{\mathrm{p}}<1\right)$. 
Table 2. The Q-Porosity Function $(\varepsilon)$ for partially porous particles $\left(0<\varepsilon_{\mathrm{p}}<1\right)$

\begin{tabular}{|c|c|c|c|c|c|c|c|c|c|c|c|c|c|c|c|c|}
\hline Particle & $\mathrm{L}$ & D & $\mathrm{V}_{\mathrm{ec}}$ & $d_{p}$ & $\mathbf{v}_{\mathrm{dp}}$ & $\mathbf{n}_{\mathrm{pq}}$ & $\gamma$ & $\mathbf{n}_{\mathrm{p}}$ & $\varepsilon_{\mathrm{p}}$ & $\left(1-\varepsilon_{0}\right)$ & $\operatorname{abs}\left(1-z_{0}\right)$ & $\varepsilon_{0}$ & $\varepsilon_{\mathrm{i}}$ & $\varepsilon_{t}$ & $\varepsilon_{\text {sk }}$ & Cont. \\
\hline Type & & & & & & & & & & & & & & & & $\left(\varepsilon_{\mathrm{t}}+\varepsilon_{\mathrm{slk}}\right)$ \\
\hline N/A & $\mathrm{cm}$ & $\mathrm{cm}$ & $\mathrm{cm}^{3}$ & $\mathrm{~cm}$ & $\mathrm{~cm}^{3}$ & none & none & none & none & none & none & none & none & none & none & none \\
\hline Solid & 10 & 0.46 & 1.66 & 0.001 & 5.E-10 & $3 . E+09$ & $2 . E+08$ & $3 . E+09$ & 0.60 & 1 & 1 & 0 & 0.6 & 0.60 & 0.40 & 1.00 \\
\hline \multirow[t]{7}{*}{ particles } & 10 & 0.46 & 1.66 & 0.001 & 5.E-10 & 3. $E+09$ & $2 . E+08$ & $2 . \mathrm{E}+09$ & 0.60 & 0.74 & 0.74 & 0.26 & 0.444 & 0.70 & 0.30 & 1.00 \\
\hline & 10 & 0.46 & 1.66 & 0.001 & 5.E-10 & $3 . E+09$ & $2 . E+08$ & $2 . E+09$ & 0.60 & 0.62 & 0.62 & 0.38 & 0.372 & 0.75 & 0.25 & 1.00 \\
\hline & 10 & 0.46 & 1.66 & 0.001 & 5.E-10 & $3 . E+09$ & $2 . E+08$ & $2 . \mathrm{E}+09$ & 0.60 & 0.6 & 0.6 & 0.4 & 0.36 & 0.76 & 0.24 & 1.00 \\
\hline & 10 & 0.46 & 1.66 & 0.001 & 5.E-10 & $3 . E+09$ & $2 . \mathrm{E}+08$ & 2.E+09 & 0.60 & 0.58 & 0.58 & 0.42 & 0.348 & 0.77 & 0.23 & 1.00 \\
\hline & 10 & 0.46 & 1.66 & 0.001 & 5.E-10 & $3 . E+09$ & $2 . E+08$ & $2 . E+09$ & 0.60 & 0.55 & 0.55 & 0.45 & 0.33 & 0.78 & 0.22 & 1.00 \\
\hline & 10 & 0.46 & 1.66 & 0.001 & 5.E-10 & $3 . E+09$ & $2 . E+08$ & $9 . E+08$ & 0.60 & 0.26 & 0.26 & 0.74 & 0.156 & 0.90 & 0.10 & 1.00 \\
\hline & 10 & 0.46 & 1.66 & 0.001 & 5.E- 10 & $3 . E+09$ & 2. $E+08$ & $2 . E+08$ & 0.60 & 0.06 & 0.06 & 0.94 & 0.04 & 0.98 & 0.02 & 1.00 \\
\hline
\end{tabular}

In our worked example shown in table 2, we show as our example a packed conduit with particles which have a particle porosity of $0.6\left(\varepsilon_{\mathrm{p}}=0.6\right)$. Because the porosity functions of $\varepsilon_{\mathrm{i}}, \varepsilon_{\mathrm{t}}$ and $\varepsilon_{\mathrm{sk}}$ are dependent on the value of $\varepsilon_{\mathrm{p}}$, we include these functions in our fig. 2 .

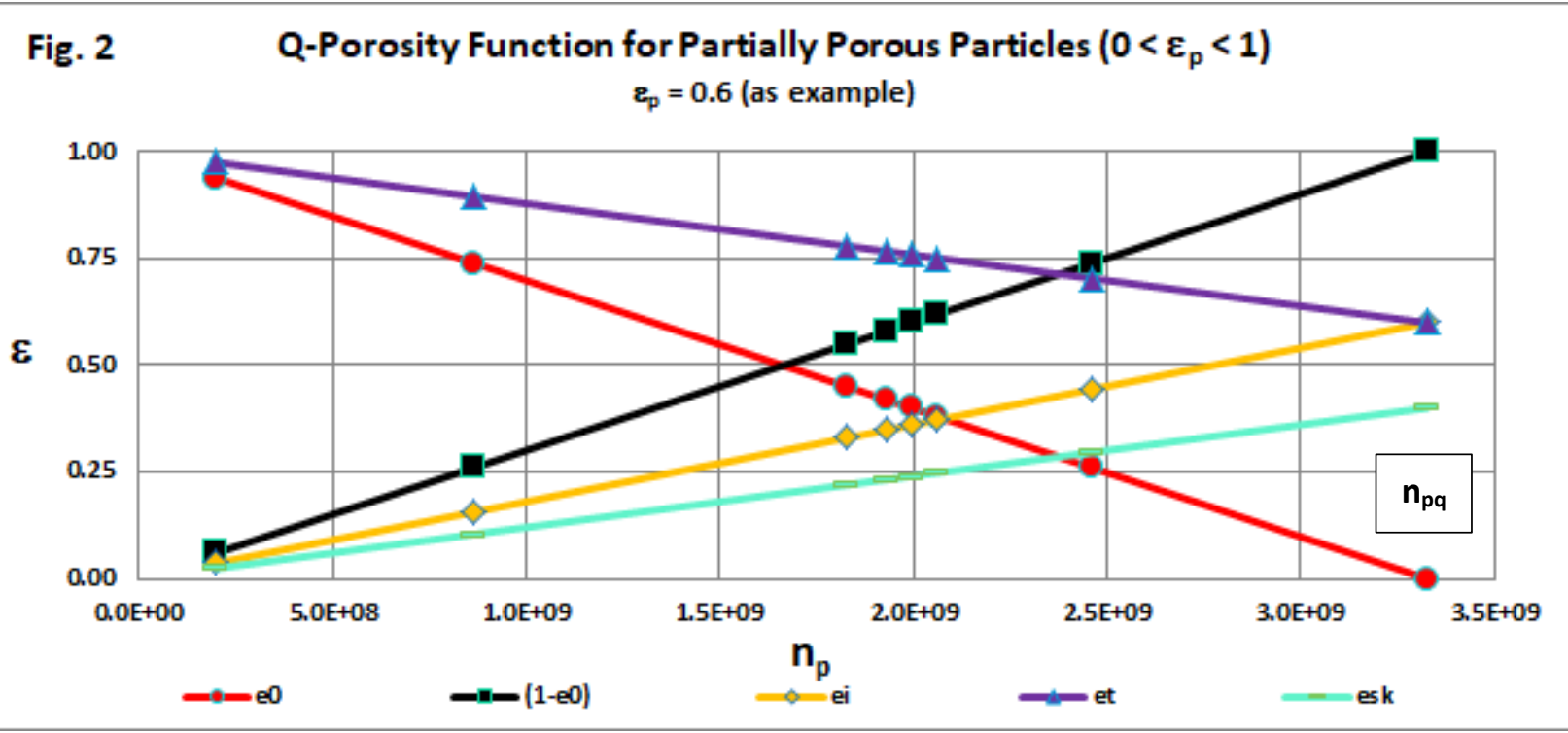

As shown in table 2 and displayed in fig. 2 , each of the 5 porosity functions, $\varepsilon_{0},\left(1-\varepsilon_{0}\right), \varepsilon_{\mathrm{i}}$, $\varepsilon_{\mathrm{t}}$, and $\varepsilon_{\mathrm{sk}}$ have discrete and different values for all values of $\mathbf{n}_{\mathbf{p}}$.

\section{b. Hypothetical Q-Particles $\left(\varepsilon_{p}=1\right)$}

2.10 We shall now consider the packing process in the special case when the particles are fully porous, i.e., they are completely made of free space $\left(\varepsilon_{\mathrm{p}}=1\right)$. This scenario is presented in Table 3. 
Table 3. The Q-Porosity Function $(\varepsilon)$ for fully porous particles $\left(\varepsilon_{\mathrm{p}}=1\right)$

\begin{tabular}{|c|c|c|c|c|c|c|c|c|c|c|c|c|c|c|c|c|}
\hline Particle & $\mathrm{L}$ & D & $\mathbf{V}_{\mathrm{ec}}$ & $d_{p}$ & $\mathbf{V}_{\mathrm{dp}}$ & $\mathbf{n}_{\mathrm{pq}}$ & $\gamma$ & $\mathbf{n}_{\mathrm{p}}$ & $\varepsilon_{p}$ & $\left(1-z_{0}\right)$ & $\operatorname{abs}\left(1-z_{0}\right)$ & $\varepsilon_{0}$ & $\varepsilon_{\mathrm{i}}$ & $\varepsilon_{\mathrm{t}}$ & $\boldsymbol{\varepsilon}_{\text {sk }}$ & Cont. \\
\hline Type & & & & & & & & & & & & & & & & $\left(\varepsilon_{\mathrm{t}}+\varepsilon_{\mathrm{sl}}\right)$ \\
\hline N/A & $\mathrm{cm}$ & $\mathrm{cm}$ & $\mathrm{cm}^{3}$ & $\mathrm{~cm}$ & $\mathrm{~cm}^{3}$ & none & none & none & none & none & none & none & none & none & none & none \\
\hline Hypothetical & 10 & 0.46 & 1.66 & 0.001 & 5.E-10 & $3 . E+09$ & $2 . E+08$ & $-2 . E+08$ & 1.00 & -0.06 & 0.06 & 1.06 & -0.06 & 1.00 & 0.00 & 1.00 \\
\hline \multirow[t]{7}{*}{ Q-particles } & 10 & 0.46 & 1.66 & 0.001 & 5.E-10 & $3 . E+09$ & $2 . E+08$ & $-9 . E+08$ & 1.00 & -0.26 & 0.26 & 1.26 & -0.26 & 1.00 & 0.00 & 1.00 \\
\hline & 10 & 0.46 & 1.66 & 0.001 & 5.E-10 & $3 . E+09$ & $2 . E+08$ & $-2 . E+09$ & 1.00 & -0.55 & 0.55 & 1.55 & -0.55 & 1.00 & 0.00 & 1.00 \\
\hline & 10 & 0.46 & 1.66 & 0.001 & 5.E-10 & $3 . E+09$ & $2 . E+08$ & $-2 . E+09$ & 1.00 & -0.58 & 0.58 & 1.58 & -0.58 & 1.00 & 0.00 & 1.00 \\
\hline & 10 & 0.46 & 1.66 & 0.001 & 5.E-10 & $3 . E+09$ & $2 . E+08$ & $-2 . E+09$ & 1.00 & -0.60 & 0.60 & 1.60 & -0.60 & 1.00 & 0.00 & 1.00 \\
\hline & 10 & 0.46 & 1.66 & 0.001 & $5 . \mathrm{E}-10$ & $3 . E+09$ & $2 . E+08$ & $-2 . E+09$ & 1.00 & -0.62 & 0.62 & 1.62 & -0.62 & 1.00 & 0.00 & 1.00 \\
\hline & 10 & 0.46 & 1.66 & 0.001 & 5.E-10 & $3 . E+09$ & $2 . E+08$ & $-2 . E+09$ & 1.00 & -0.74 & 0.74 & 1.74 & -0.74 & 1.00 & 0.00 & 1.00 \\
\hline & 10 & 0.46 & 1.66 & 0.001 & 5.E-10 & 3. $E+09$ & 2. $E+08$ & $-3 . E+09$ & 1.00 & -1.00 & 1.00 & 2.00 & -1.00 & 1.00 & 0.00 & 1.00 \\
\hline
\end{tabular}

As shown in table 3, our packing process for particles made of free space $\left(\varepsilon_{\mathrm{p}}=1\right)$, which we designate as hypothetical Q-particles, is represented by increasingly negative values of $\mathbf{n}_{\mathbf{p}}$. Accordingly, as shown in fig. 3A the domain of the Q-Porosity function runs from 0 to $-\mathrm{n}_{\mathrm{pq}}$. Similarly, it follows that the range of the function varies between the values of -1 and 2 , as shown in table 3 and also in fig. $3 \mathrm{~A}$.

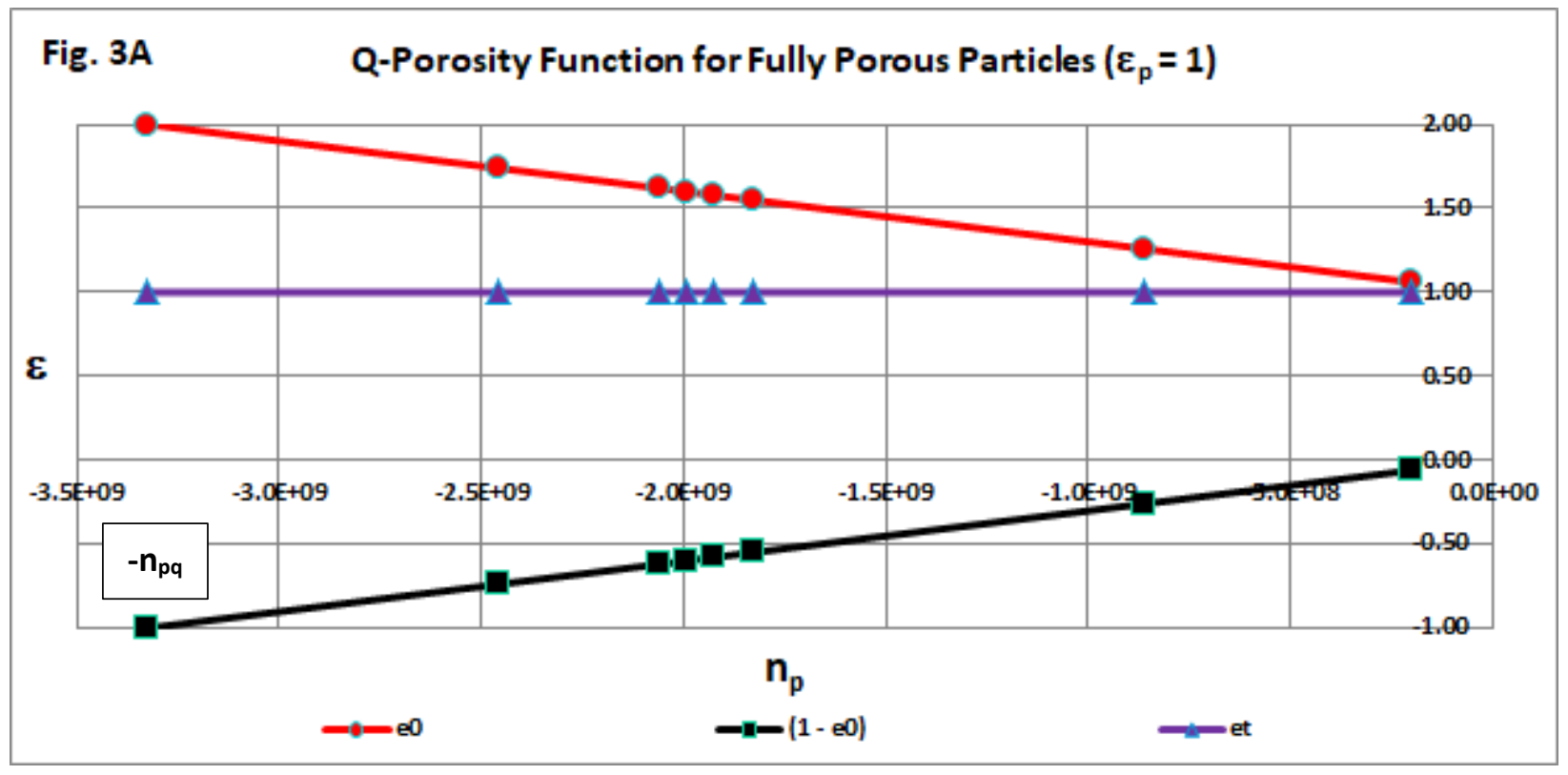

In this scenario the Q-Porosity functions $\varepsilon_{\mathrm{t}}=1$ and $\varepsilon_{\mathrm{sk}}=0$ for all values of $\mathbf{n}_{\mathbf{p}}$. The function $\varepsilon_{\mathrm{i}}$ has identical values to the function $\left(1-\varepsilon_{0}\right)$ and varies between 0 and -1 , whereas the value of the function $\varepsilon_{0}$ varies between 1 and 2 .

Let us now define the directional component of packing a conduit with hypothetical Q-particles in terms of our mathematical Q-Porosity functions. As we move along the $\mathrm{x}$ axis of fig. $3 \mathrm{~A}$ in the direction of right to left, starting at the origin of the plot at $\mathbf{n}_{\mathbf{p}}=0$, the corresponding values on the $y$ axis represent the changing characteristics of the Q-Porosity function $\varepsilon$ in the filling 
(packing) process. This direction of filling is the opposite of that for solid particles. At the starting point of $\mathbf{n}_{\mathbf{p}}=0$, we consider the conduit to be devoid of all particles (including particles of free space) and at the maximum value of $\mathbf{n}_{\mathbf{p}}=-\mathrm{n}_{\mathrm{pq}}$ achieved in the filling process, the conduit is fully packed with particles of free space (hypothetical Q-particles). Accordingly, filling of a "packed" conduit with hypothetical Q-particles is represented by increasing negative values of $\mathbf{n}_{\mathbf{p}}$, i.e., the motion right to left along the $\mathrm{x}$ axis of the plot starting at $\mathbf{n}_{\mathbf{p}}=0 \leftarrow$.

It follows that in the case of hypothetical Q-particles which are made of free space, Kepler's conjecture does not apply, since particles of free space are mutually inclusive with free space, i.e., they are free space. Accordingly, the maximum value of $\mathbf{n}_{\mathbf{p}}$ achieved empirically is $-\mathbf{n}_{\mathrm{pq}}$, which corresponds to the conduit being filled with free space, and is also the upper theoretical limit of $\mathbf{n}_{\mathbf{p}}$ in these circumstances.

We choose to use, advantageously, the absolute value of the porosity function $\left(1-\varepsilon_{0}\right)$ in our theoretical development, which represents the magnitude of the function and is, therefore, always positive, as shown in fig. 3B.

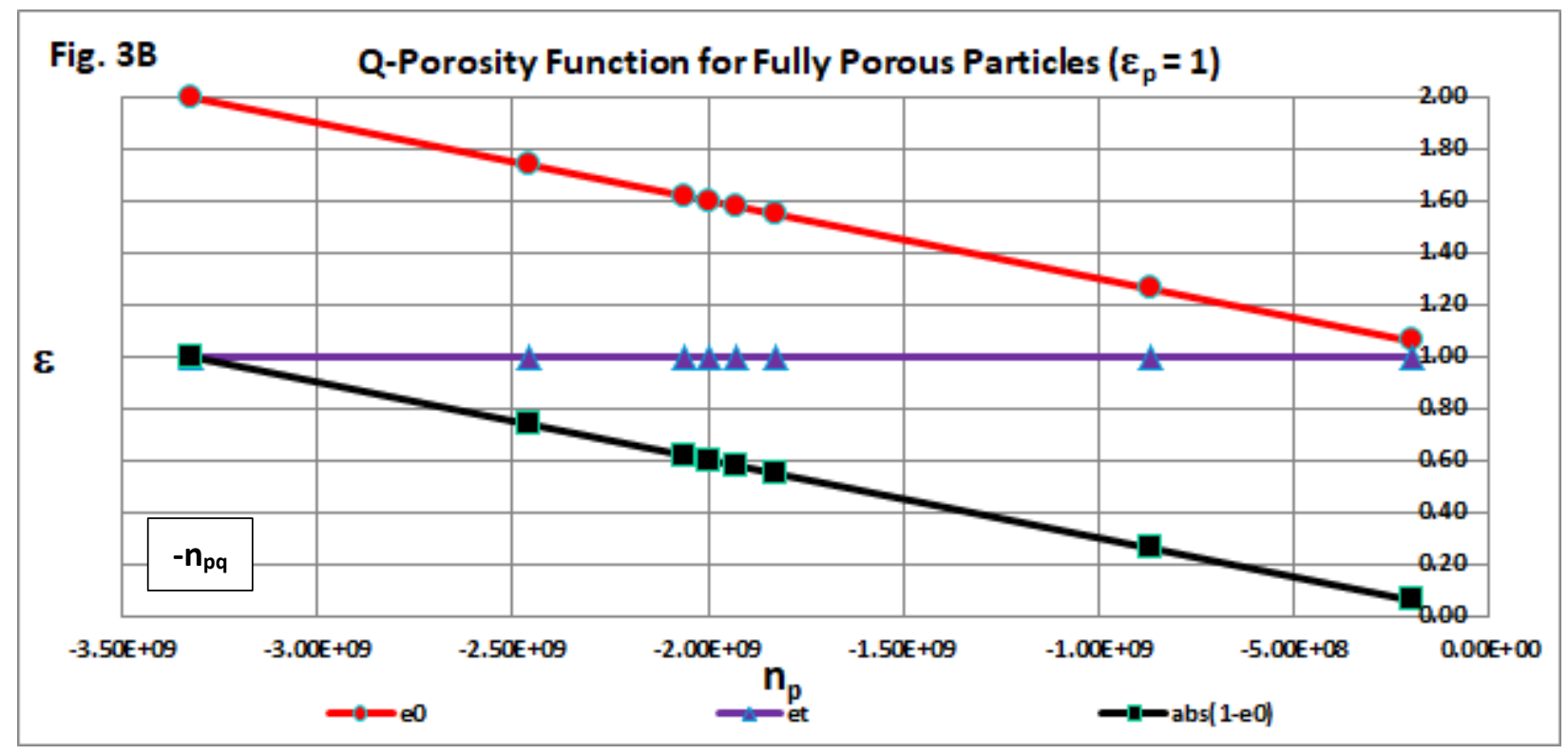

Accordingly, as shown in fig. 3B, the ranges of all Q-Porosity functions of interest in this special case have positive magnitudes, and vary between the values of 0 and 2 .

\section{E. The Packed Conduit Hypothetical Q Channel Defined}

3.10 Let us define a hypothetical cylindrical fluid channel within a packed conduit, which we shall call the hypothetical Q channel (HQC), whose characteristic dimensions are defined as:

$$
\mathrm{d}_{\mathrm{c}}=\underset{\operatorname{abs}\left(1-\varepsilon_{0}\right)}{\underline{\mathrm{d}_{\mathrm{p}}}}=\frac{\underline{\mathrm{d}_{\mathrm{p}}}}{\operatorname{abs}\left(\mathrm{n}_{\mathrm{p}} / \mathrm{n}_{\mathrm{pq}}\right)}
$$


Where, $d_{c}=$ the diameter of the HQC.

It follows that we may write [see Eqs. (7) and (14) ]:

$$
\mathrm{v}_{\mathrm{c}}=\mathrm{V}_{\mathrm{ec}} \varepsilon_{\mathrm{t}}=\frac{\pi \mathrm{n}_{\mathrm{pq}}}{6} \underline{\mathrm{d}}_{\mathrm{p}}{ }^{3} \varepsilon_{\mathrm{t}}
$$

Where, $\mathrm{v}_{\mathrm{c}}=$ the volume of the HQC.

It follows that we may also write:

$$
\mathrm{a}_{\mathrm{c}}=\frac{\pi \mathrm{d}_{\mathrm{c}}^{2}}{4}=\frac{\pi \mathrm{n}_{\mathrm{pq}}^{2} \mathrm{~d}_{\mathrm{p}}^{2}}{4 \mathrm{n}_{\mathrm{p}}^{2}}
$$

Where, $\mathrm{a}_{\mathrm{c}}=$ the cross sectional area of the HQC.

Similarly, we may write:

$$
1_{\mathrm{c}}=\underset{\mathrm{a}_{\mathrm{c}}}{\underline{\mathrm{v}}_{\mathrm{c}}}=\frac{2 \mathrm{n}_{\mathrm{p}}{ }^{2} \underline{\mathrm{d}}_{\mathrm{p}} \underline{\varepsilon_{\mathrm{t}}}}{3 \mathrm{n}_{\mathrm{pq}}}
$$

Where, $1_{c}=$ the length of the HQC.

3.11 Let us define the Unit Hypothetical Q Channel as a special case of the more general HQC. It is defined as a conduit filled with hypothetical Q-particles having two fixed boundary conditions: (1), $\mathrm{d}_{\mathrm{p}}=\mathrm{D}$ and $(2), \mathrm{n}_{\mathrm{p}}=-\mathrm{n}_{\mathrm{pq}}$.

It follows that, since the function $\varepsilon_{0}=2$ when $n_{p}=-n_{p q}$, the function $\operatorname{abs}\left(1-\varepsilon_{0}\right)=1=\varepsilon_{t}$.

Accordingly, any "empty" conduit/capillary is represented in the QFFM by what we now term

\begin{tabular}{|c|c|c|c|c|c|c|c|c|c|c|c|c|c|c|c|c|c|c|c|c|c|c|}
\hline Particle & $\mathrm{L}$ & D & $\mathrm{v}_{\mathrm{ec}}$ & $d_{p}$ & $\mathbf{v}_{\mathrm{dp}}$ & $\mathbf{n}_{\mathrm{pq}}$ & $\gamma$ & $\mathbf{n}_{\mathrm{p}}$ & $\varepsilon_{\mathrm{p}}$ & $\left(1-z_{0}\right)$ & $\operatorname{abs}\left(1-\varepsilon_{0}\right)$ & $\varepsilon_{0}$ & $\varepsilon_{i}$ & $\varepsilon_{t}$ & $\varepsilon_{\text {sk }}$ & Cont. & $\delta$ & $\tau$ & $d_{c}$ & $a_{c}$ & lc & $\mathbf{v}_{\mathrm{c}}$ \\
\hline Type & & & & & & & & & & & & & & & & $\left(\varepsilon_{t}+\varepsilon_{\text {st: }}\right)$ & & & & & & \\
\hline N/A & $\mathrm{cm}$ & $\mathrm{cm}$ & $\mathrm{cm}^{3}$ & $\mathrm{~cm}$ & & none & none & none & none & none & none & none & none & none & none & & none & none & $\mathrm{cm}$ & & $\mathrm{cm}$ & $\mathrm{cm}^{3}$ \\
\hline Hypothetical & 10 & 0.46 & 1.66 & 0.46 & 0.05 & 32.61 & 1.50 & -1.00 & 1.00 & -0.03 & 0.03 & 1.03 & -0.03 & 1.00 & 0.00 & 1.00 & 0.913 & 1.370 & 15.00 & 176.79 & 0.01 & 1.66 \\
\hline Q-particles & 10 & 0.46 & 1.66 & 0.46 & 0.05 & 32.61 & 1.50 & -5.00 & 1.00 & -0.15 & 0.15 & 1.15 & -0.15 & 1.00 & 0.00 & 1.00 & 0.652 & 0.978 & 3.00 & 7.07 & 0.24 & 1.66 \\
\hline$d p=D$ & 10 & 0.46 & 1.66 & 0.46 & 0.05 & 32.61 & 1.50 & -10.00 & 1.00 & -0.31 & 0.31 & 1.31 & -0.31 & 1.00 & 0.00 & 1.00 & 0.448 & 0.672 & 1.50 & 1.77 & 0.94 & 1.66 \\
\hline \multirow[t]{5}{*}{$\mathrm{np}=-\mathrm{npq}$} & 10 & 0.46 & 1.66 & 0.46 & 0.05 & 32.61 & 1.50 & -15.00 & 1.00 & -0.46 & 0.46 & 1.46 & -0.46 & 1.00 & 0.00 & 1.00 & 0.321 & 0.482 & 1.00 & 0.79 & 2.12 & 1.66 \\
\hline & 10 & 0.46 & 1.66 & 0.46 & 0.05 & 32.61 & 1.50 & -20.00 & 1.00 & -0.61 & 0.61 & 1.61 & -0.61 & 1.00 & 0.00 & 1.00 & 0.238 & 0.357 & 0.75 & 0.44 & 3.76 & 1.66 \\
\hline & 10 & 0.46 & 1.66 & 0.46 & 0.05 & 32.61 & 1.50 & -25.00 & 1.00 & -0.77 & 0.77 & 1.77 & -0.77 & 1.00 & 0.00 & 1.00 & 0.181 & 0.272 & 0.60 & 0.28 & 5.88 & 1.66 \\
\hline & 10 & 0.46 & 1.66 & 0.46 & 0.05 & 32.61 & 1.50 & -30.00 & 1.00 & -0.92 & 0.92 & 1.92 & -0.92 & 1.00 & 0.00 & 1.00 & 0.141 & 0.212 & 0.50 & 0.20 & 8.46 & 1.66 \\
\hline & 10 & 0.46 & 1.66 & 0.46 & 0.05 & 32.61 & 1.50 & -32.61 & 1.00 & -1.00 & 1.00 & 2.00 & -1.00 & 1.00 & 0.00 & 1.00 & 0.125 & 0.188 & 0.46 & 0.17 & 10.00 & 1.66 \\
\hline
\end{tabular}
the Unit HQC since, by definition, its Q-Porosity functions of [abs $\left.\left(1-\varepsilon_{0}\right)\right]$ and $\varepsilon_{\mathrm{t}}$ are unity, as shown below in table 4 for our worked example.

Table 4. The Unit Hypothetical Q Channel 
As shown in table 4, any conduit/capillary when totally filled with hypothetical Q-particles $\left(\mathrm{n}_{\mathrm{p}}=\right.$ $\left.-\mathrm{n}_{\mathrm{pq}}\right)$, whose diameters are equivalent to the diameter of the conduit $\left(\mathrm{d}_{\mathrm{p}}=\mathrm{D}=\mathrm{d}_{\mathrm{c}}\right)$, will always have the constant values shown below, regardless of what the conduit dimensions are.

Thus, an empty conduit/capillary is defined in the QFFM as a packed conduit containing hypothetical particles with a particle porosity of unity $\left(\varepsilon_{p}=1\right)$, and is represented by the Unit HQC with the following constant values:

$\varepsilon_{\mathrm{p}}=1 ; \mathrm{n}_{\mathrm{p}}=-\mathrm{n}_{\mathrm{pq}} ; \mathrm{d}_{\mathrm{p}}=\mathrm{D}=\mathrm{d}_{\mathrm{c}} ; \mathrm{l}_{\mathrm{c}}=\mathrm{L} ; \mathrm{v}_{\mathrm{c}}=\mathrm{V}_{\mathrm{ec}} ; \operatorname{abs}\left(1-\varepsilon_{0}\right)=1 ; \varepsilon_{0}=2 ; \varepsilon_{\mathrm{t}}=1 ; \delta=0.125(1 / 8) ; \gamma=1.5$ $(3 / 2) ; \tau=0.188(3 / 16)$.

3.12 To further articulate the characteristics of the Unit HQC in the case of our chosen worked example, we present here 4 graphical representations of the primary channel functions; see figs. 4A, 4B, 4C and 4D.

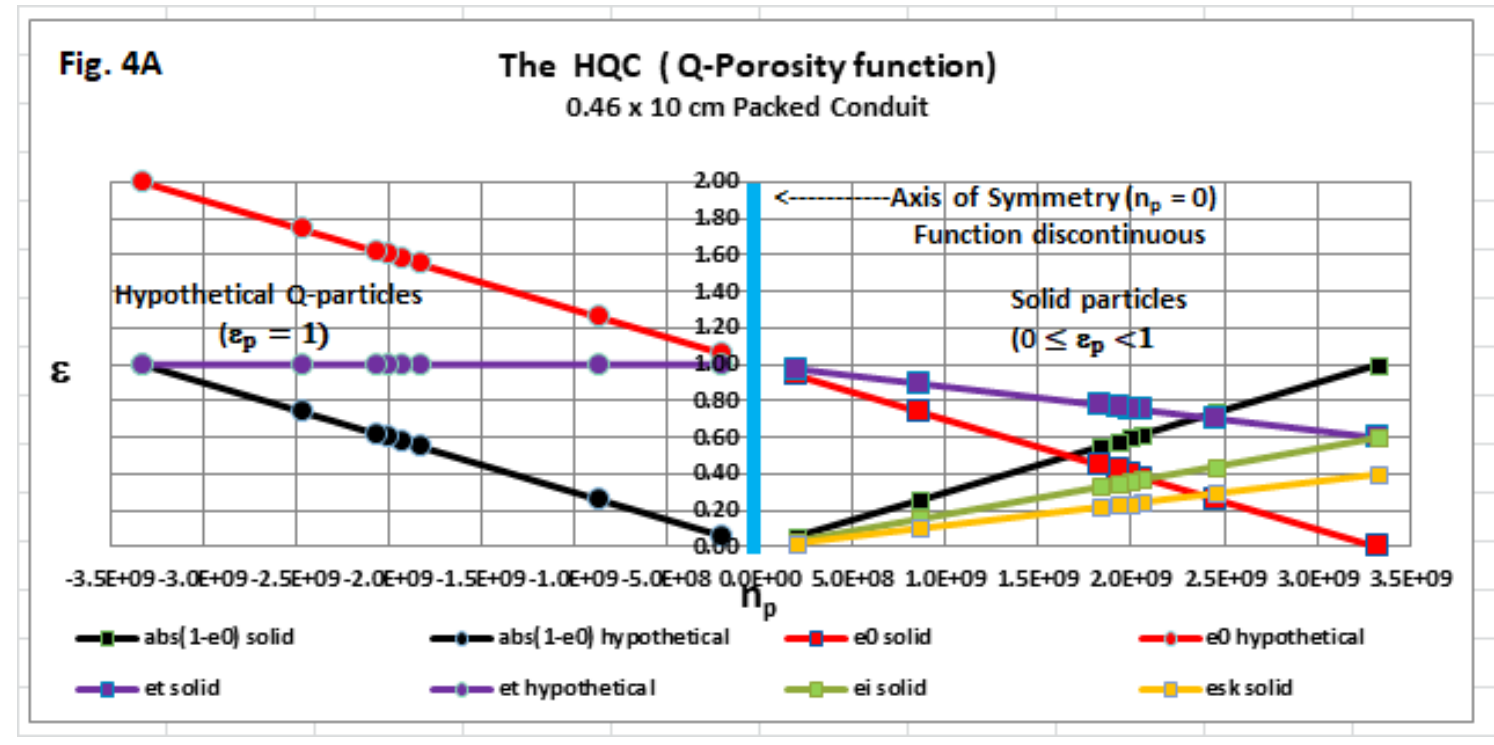

Fig. 4B

The HQC ( $d_{c}$ function)

$0.46 \times 10 \mathrm{~cm}$ Packed Conduit

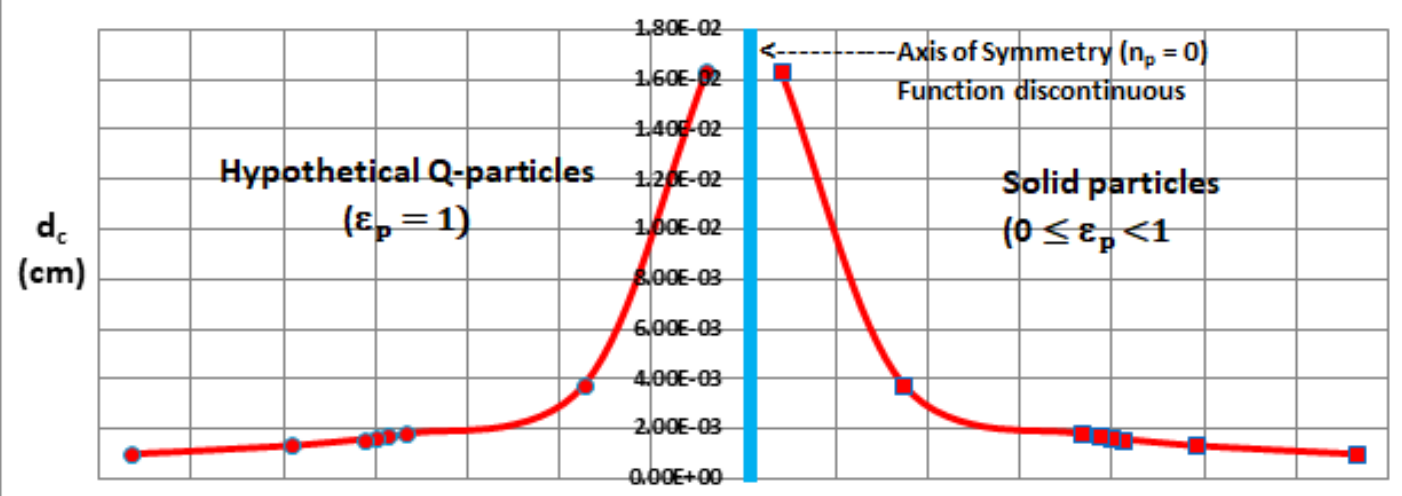

$-3.5 E+09-3.0 E+09-2.5 E+09-2.0 E+09-1.5 E+09-1.0 E+09-5.0 E+080.0 E+00 \quad 5.0 E+08 \quad 1.0 E+091.5 E+092.0 E+092.5 E+093.0 E+093.5 E+09$ 

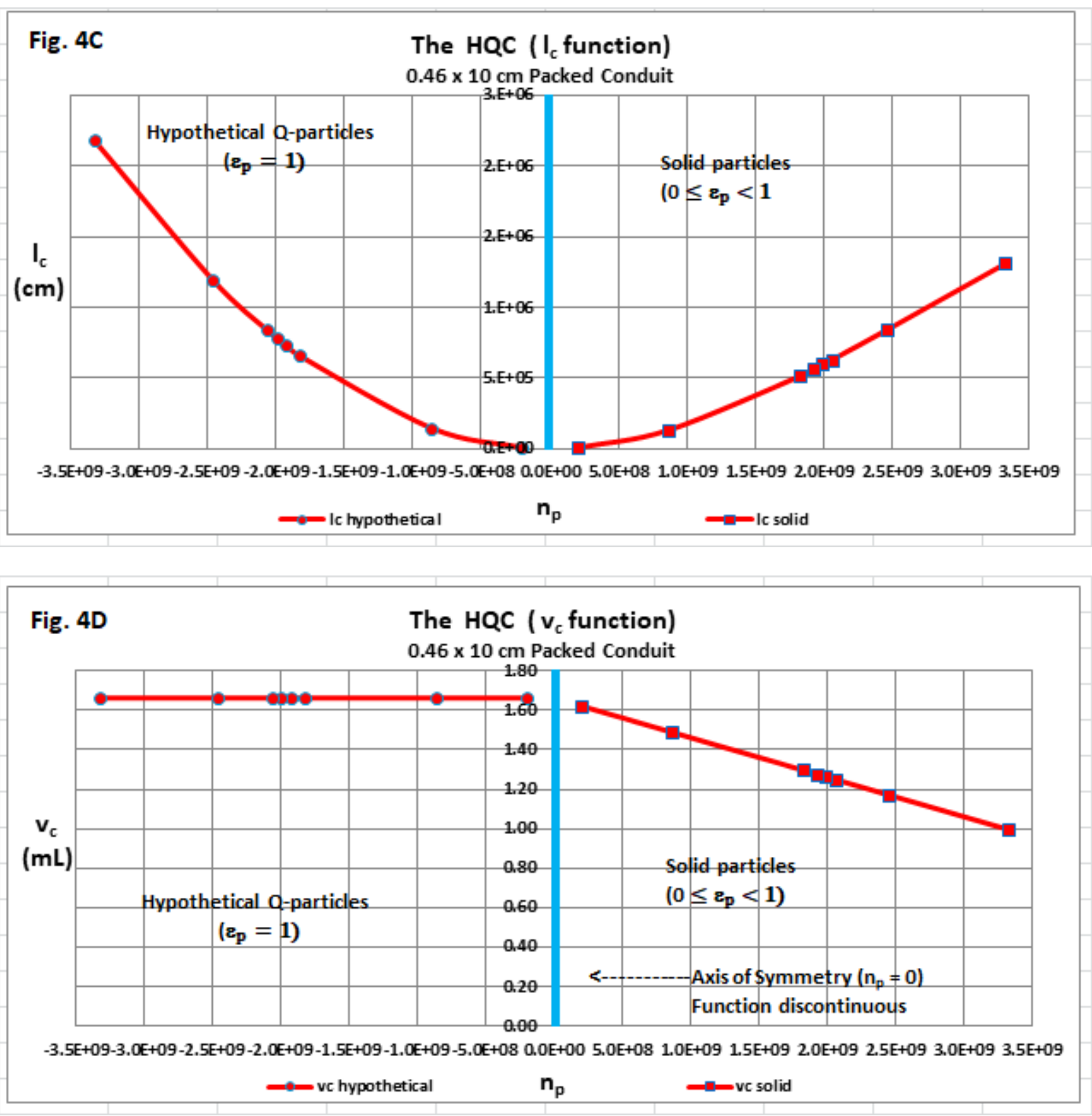

As shown in fig. 4A, the vertical line in the range of the Q-Porosity function $(\varepsilon)$, r.e., $\mathrm{n}_{\mathrm{p}}=0$, represents the axis of symmetry between the half-plane Q-Porosity function for solid particles, on the one hand (right hand side half-plane), and hypothetical Q particles, on the other hand (left hand side half-plane). Note that the Q-Porosity functions are discontinuous at the value of $\mathrm{n}_{\mathrm{p}}=0$, but are continuous at all other values of $\mathbf{n}_{\mathrm{p}}$, i.e., $-\mathrm{n}_{\mathrm{pq}} \leq \mathrm{n}_{\mathrm{p}}<0 ; 0<\mathrm{n}_{\mathrm{p}} \leq \mathrm{n}_{\mathrm{pq}}$.

It follows that, as shown in fig. $4 \mathrm{~B}$, the HQC function $\mathrm{d}_{\mathrm{c}}$ is correspondingly discontinuous at the value of $n_{p}=0$, since at this precise value the diameter of the HQC tends to infinity. Thus, in the QFFM, the "infinite diameter packed conduit" is prohibited by hypothesis. 
Finally, it follows that, as shown in fig. $4 \mathrm{C}$, the HQC function $1_{c}$ is zero at the value of $n_{p}=0$. In addition, the value of $l_{c}$ is always greater for values of $n_{p}$ less than zero, than for values of $n_{p}$ greater than zero, a consequence of a value for external porosity in excess of unity, in this halfplane of the function.

Similarly, it follows that, as shown in fig. 4D, the HQC function $\mathrm{v}_{\mathrm{c}}$ is correspondingly discontinuous at the value of $n_{p}=0$, since at this precise value, the volume of the HQC is undefined, but at all values of $\mathrm{n}_{\mathrm{p}}$ less than zero, its volume is a constant positive value and at all values of $\mathrm{n}_{\mathrm{p}}$ greater than zero, it has a variable positive value.

\section{F. Fluid motion in the HQC}

\section{Physical Reality underlying the HQC}

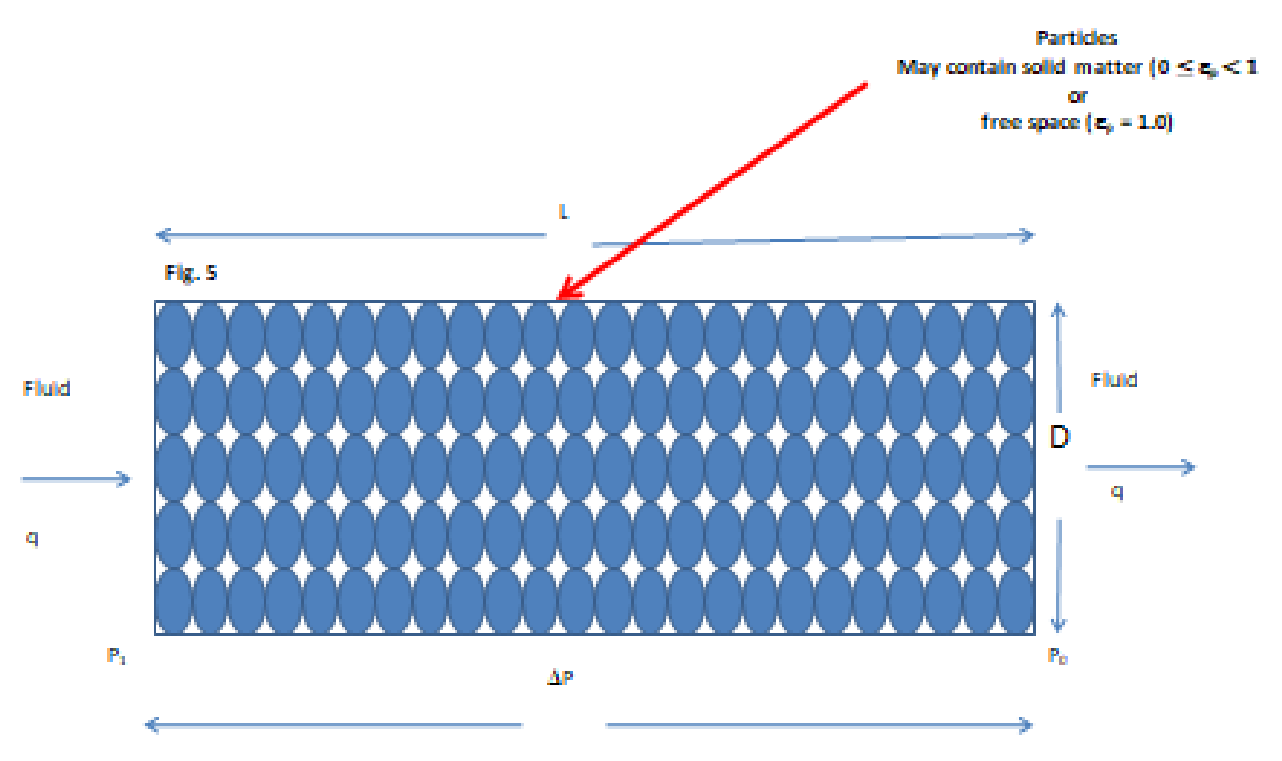

4.10 Let us consider the motion of the fluid in the HQC as a control volume element of fluid flowing through a packed conduit under study with a volumetric flow rate, q; fluid absolute viscosity $\eta$; fluid density $\rho_{\mathrm{f}}$, and under a differential pressure, $\Delta \mathrm{P}$, defined as:

$$
\Delta \mathrm{P}=\mathrm{P}_{1}-\mathrm{P}_{0}
$$

Where, $\Delta \mathrm{P}=$ the differential pressure drop across the packed conduit; $\mathrm{P}_{1}=$ hydraulic pressure at conduit inlet; $\mathrm{P}_{0}=$ hydraulic pressure at conduit outlet. [See Fig. 5]. 
We shall now develop the interrelationships among the conduit parameters which characterize the motion of the fluid within the HQC. In so doing, we derive a unique relationship between the driving force for fluid flow, which is known generally as the pressure gradient, and other HQC variables.

4.11 Let $Q$ be the position at any time $t$ of a point describing a circle of radius a with uniform angular velocity $\omega$ about the center $\mathrm{O}$. Let $\mathrm{P}$ be the projection of $\mathrm{Q}$ on any diameter A'OA. Let the angle $\mathrm{AOQ}=\Phi$. And let $\mathrm{OP}=\mathrm{x}$. [See Fig. 5B].

Fig. 5B

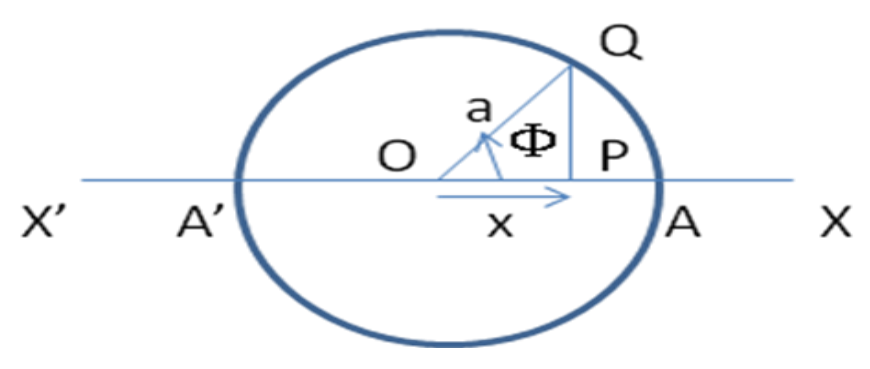

Thus:

$$
\frac{\mathrm{d}}{\mathrm{dt}}(\Phi)=\omega
$$

where $\omega$ is a constant

Hence,

$$
\Phi=(\omega t+\alpha)
$$

where $\omega$ and $\alpha$ are constants

Hence,

$$
\mathrm{x}=\mathrm{a} \cos \Phi=\mathrm{a} \cos (\omega \mathrm{t}+\alpha)
$$

In Eq. (32) below, we shall define the meaning of each of the flow terms in Eq. (30) as they relate to the parameters of the packed conduit embodied in the concept of the HQC.

4.12 We begin with the hypothesis that the motion of the fluid within the HQC is defined by that of uniform circular motion and, accordingly, the general equation of Simple Harmonic Motion (SHM), represented by Equation (30); 
Where, $\Phi=$ the phase of the motion; $\omega=$ the frequency of the motion; $\mathrm{t}=$ the elapsed time of the motion; $\alpha=$ the epoch of the motion, all as taught in the general theory of classical mechanics [25].

4.13 Let $\Phi=\mathrm{P}_{\mathrm{Q}} ; \omega=\lambda / \phi_{\mathrm{h}} ; \mathrm{t}=\mathrm{Q}_{\mathrm{N}} ; \alpha=2 \pi \mathrm{k}_{1} / 360 ;$ and $\mathrm{q}=\mathrm{d} V / \mathrm{dt}$.

Where, $\mathrm{P}_{\mathrm{Q}}=$ instantaneous HQC drag normalized viscous friction factor; $\omega=$ instantaneous HQC fluid resistance; $\lambda=$ instantaneous HQC wall-adjusted fluid current normalization coefficient; $\phi_{\mathrm{h}}=$ drag normalized hydraulic channel circumference; $\mathrm{Q}_{\mathrm{N}}=$ instantaneous fluid current; $\mathrm{k}_{1}=$ the drag normalized wetted surface area; and $V=$ a control volume of fluid.

For clarity here, in our modeling of the fluid motion in the HQC, we recite our procedure of equating normalized dimensionless parameters related to the HQC to dimensional parameters in our SHM model. Thus, for instance, the dimensionless parameter $Q_{N}$ of the HQC is equated to the elapsed time parameter $\mathrm{t}$, of the SHM model, which has the dimensional units of seconds.

Note also that we have arbitrarily chosen the units of radians in our definition of the epoch of the motion in the SHM model, by virtue of the unit conversion $(2 \pi / 360)$, which is the conversion factor between degrees and radians.

Thus, substituting for the terms which define our fluid motion in the HQC, we may now rewrite Equation (30) as follows:

$$
\mathrm{P}_{\mathrm{Q}}=\left(\mathrm{k}_{1}+\lambda \mathrm{Q}_{\mathrm{N}} / \phi_{\mathrm{h}}\right)
$$

It follows that we may state that, in the limit as $\mathrm{Q}_{\mathrm{N}}$ tends to zero, the value of $\mathrm{P}_{\mathrm{Q}}$ approaches the constant value of $\mathrm{k}_{1}$. We can express this algebraically as:

$$
\underset{\operatorname{Lim} \mathrm{Q}_{\mathrm{N}} \rightarrow 0}{\mathrm{P}_{\mathrm{Q}}}=\mathrm{k}_{1}
$$

Thus, the function $\mathrm{P}_{\mathrm{Q}}$ is bounded only on one side and varies from a minimum value of $\mathrm{k}_{1}$ on the low side, when the fluid is at rest $\left(\mathrm{Q}_{\mathrm{N}}=0\right)$, and is unbounded on the high side (high value of $\mathrm{Q}_{\mathrm{N}}$ ).

4.14 Let us define a corresponding HQC kinetic friction factor as:

$$
\mathrm{P}_{\mathrm{K}}=\underline{\mathrm{P}_{\mathrm{Q}}}
$$

Where, $\mathrm{P}_{\mathrm{K}}=$ the instantaneous $\mathrm{HQC}$ normalized kinetic friction factor. 
Thus, the function $\mathrm{P}_{\mathrm{K}}$ is infinite when the fluid is at rest $\left(\mathrm{Q}_{\mathrm{N}}=0\right)$.

4.15 Driven by the principle of avoiding non-finite boundaries, let us define the reciprocal of Equation (33) as:

$$
\frac{1}{P_{K}}=\frac{Q_{N}}{P_{Q}}=\Theta
$$

Where, $\Theta=$ the dimensionless permeability of the HQC.

It follows that we may write:

$$
\Theta=\frac{1}{\left(\mathrm{k}_{1} / \mathrm{Q}_{\mathrm{N}}+\lambda / \phi_{\mathrm{h}}\right)}
$$

It further follows that we may now state that, in the limit, as the value of $Q_{N}$ tends to infinity:

$$
\underset{\left(\operatorname{Lim} Q_{N} \rightarrow \infty\right)}{\Theta=\frac{\phi_{h}}{\lambda}}
$$

Similarly, we may state that, in the limit, as $Q_{N}$ tends to zero:

$$
\begin{array}{r}
\Theta=0 \\
\left(\operatorname{Lim} Q_{N} \rightarrow 0\right)
\end{array}
$$

Thus, the dimensionless permeability function of the HQC, $\Theta$, is finite on both sides and varies between the minimum limit of 0 on the low side and the maximum limit of $\phi_{\mathrm{h}} / \lambda$, on the high side.

The dimensionless parameters of the HQC are shown in fig. 6 in a log-log plot against $\mathrm{Q}_{\mathrm{N}}$.

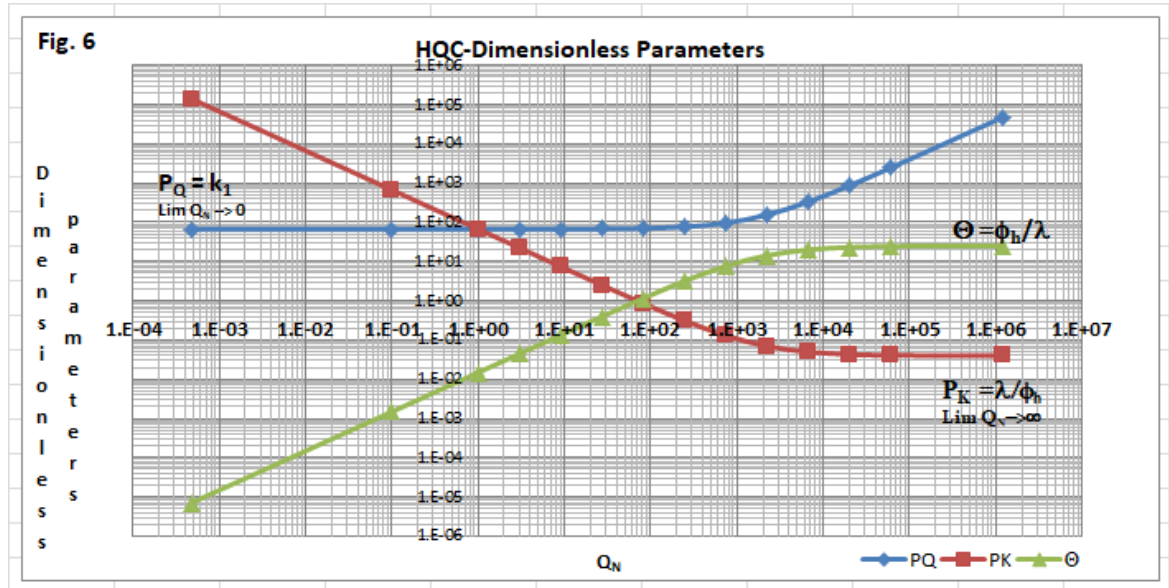


4.16 Let us now define $\mathrm{k}_{2}$, the drag normalized hydraulic channel perimeter, a normalization coefficient, as:

$$
\mathrm{k}_{2}=\frac{1}{2 \pi \mathrm{r}_{\mathrm{h}}}
$$

and

$$
\phi_{\mathrm{h}}=2 \pi \mathrm{r}_{\mathrm{h}}
$$

and

$$
\mathrm{r}_{\mathrm{h}}=\frac{\mathrm{SA}_{\mathrm{p}}}{\mathrm{CSA}_{\mathrm{p}}}=4
$$

Where, $\mathrm{k}_{2}=$ fluid motion dynamic constant; $\mathrm{r}_{\mathrm{h}}=$ the normalization coefficient of fluid drag.

4.17 Let us now define $\mathrm{k}_{1}$, the drag normalized wetted surface area, a normalization coefficient, as:

$$
\mathrm{k}_{1}=\frac{4 \pi \mathrm{r}^{2}}{3}=\frac{64 \pi}{3}
$$

It follows that we may now rewrite Equation (32) as:

$$
\mathrm{P}_{\mathrm{Q}}=\frac{64 \pi}{3}+\frac{\lambda \mathrm{Q}_{\mathrm{N}}}{8 \pi}
$$

4.18 Let us define $\delta$, a porosity normalization coefficient, as:

$$
\delta=\underline{1} \underline{\varepsilon}_{0}^{3}
$$

4.19 Let us define $\tau$, a tortuosity normalization coefficient, as:

$$
\tau=\delta \gamma
$$


4.20 Let us now define $Q_{N}$, the instantaneous fluid current, a dimensionless parameter, as:

$$
\mathrm{Q}_{\mathrm{N}}=\delta \mathrm{R}_{\mathrm{em}}
$$

Where, $\mathrm{R}_{\mathrm{em}}=$ the modified Reynolds number, defined below in Eqs. (54), (55) and (57).

4.21 Let us now define $\lambda$, the HQC wall-adjusted fluid current normalization coefficient, as:

$$
\lambda=\left(1+\mathrm{W}_{\mathrm{N}}\right)
$$

Where, $\mathrm{W}_{\mathrm{N}}=$ the net wall effect, and is restricted to where $0 \leq \mathrm{W}_{\mathrm{N}}$

It follows that we may now rewrite Equation (40) as:

$$
\mathrm{P}_{\mathrm{Q}}=\frac{64 \pi}{3}+\frac{\delta \lambda \mathrm{R}_{\mathrm{em}}}{2 \pi \mathrm{r}_{\mathrm{h}}}
$$

Substituting for $\lambda$ in Equation (45), gives:

$$
\mathrm{P}_{\mathrm{Q}}=\frac{64 \pi}{3}+\frac{\delta\left(1+\mathrm{W}_{\mathrm{N}}\right) \mathrm{R}_{\mathrm{em}}}{2 \pi \mathrm{r}_{\mathrm{h}}}
$$

4.22 Let us further refine the definition of $\mathrm{W}_{\mathrm{N}}$ as:

$$
\mathrm{W}_{\mathrm{N}}=\mathrm{W}_{1}+\mathrm{W}_{2 \mathrm{R}}
$$

Where, $\mathrm{W}_{1}=$ the primary wall effect, defined below; $\mathrm{W}_{2 \mathrm{R}}=$ the residual secondary wall effect, defined below.

4.23 Let us define $\omega_{0}$, the dimensionless resistance of the fluid in the absence of any wall effect $(\lambda=1)$, i.e., $\mathrm{W}_{\mathrm{N}}=0$, as follows:

$$
\omega_{0}=\frac{1}{\phi_{\mathrm{h}}}
$$


4.24 Let us define $\beta_{0}$, the dimensionless fluid/wall boundary layer in the absence of wall effect ( $\lambda$ $=1$ ), i.e., $\mathrm{W}_{\mathrm{N}}=0$, as follows:

$$
\beta_{0}=\frac{\mathrm{k}_{1}}{\left(\omega_{0} \mathrm{Q}_{\mathrm{N}}+\mathrm{k}_{1}\right)} \quad=\frac{\mathrm{k}_{1}}{\left(\mathrm{Q}_{\mathrm{N}} / \phi_{\mathrm{h}}+\mathrm{k}_{1}\right)}
$$

4.25 Let us define $\mathrm{W}_{1}$, the dimensionless boundary layer component of fluid current, which we will refer to as the primary wall effect, as follows:

$$
\mathrm{W}_{1}=\underline{\beta}_{\tau}{ }^{(1 / 3)}
$$

4.26 Let us define $\mathrm{k}_{\mathrm{dc}}$, the channel relative wall surface roughness coefficient, as follows:

$$
\mathrm{k}_{\mathrm{dc}}=\underline{\mathrm{k}}
$$

where, $\mathrm{k}$ is a measure of wall roughness.

4.27 Let us define $\mathrm{W}_{2}$, the wall roughness component of fluid current, which we will refer to as the secondary wall effect, as follows:

$$
\mathrm{W}_{2}=30 \mathrm{k}_{\mathrm{dc}}{ }^{(1 / 3)}
$$

Note that the value of 30 appearing in Equation (52) is not based upon any fundamentally derived concept but rather on our empirical data. We are confident, however, that this value has to do with end-effects of the packed conduit but have not as of this writing been able to define it in more precise terms.

4.28 Let us define $\mathrm{W}_{2 \mathrm{R}}$, the residual wall roughness component of fluid current, as follows:

$$
\mathrm{W}_{2 \mathrm{R}}=\mathrm{W}_{2}-\mathrm{W}_{1}{ }^{1.2}
$$

We pause here, yet again, to explain the origin of the exponent of 1.2 appearing in Equation (53). Because we have based our definition of the boundary layer on a theoretical asymptote, i.e., $\lambda=1$, which is never precisely the case in real life, we have included this exponent value as a correction factor to account for this assumption in our theoretical model. 
4.29 Let us define the modified Reynolds number as:

$$
\mathrm{R}_{\mathrm{em}}=\underline{\mathrm{n}}_{\mathrm{k}}
$$

Where, $\mathrm{n}_{\mathrm{k}}=$ the kinetic hydraulic force exerted per unit element of fluid control volume; $\mathrm{n}_{\mathrm{v}}=$ the viscous hydraulic force exerted per unit element of fluid control volume.

4.30 Let $\mathrm{n}_{\mathrm{k}}$, the kinetic hydraulic force per unit element of fluid control volume, be defined as:

$$
\mathrm{n}_{\mathrm{k}}=\frac{\delta \mu_{\mathrm{s}}^{2} \rho_{\mathrm{f}}}{\mathrm{d}_{\mathrm{c}}}
$$

Where, $\mu_{\mathrm{s}}=$ fluid superficial velocity, which, in turn, is defined as:

$$
\mu_{\mathrm{s}}=\frac{4 \mathrm{q}}{\pi \mathrm{D}^{2}}
$$

4.31 In a dimensional analysis using the cgs convention, we demonstrate the dimensional integrity of $n_{k}$, thusly;

$$
\begin{aligned}
\mathrm{n}_{\mathrm{k}}=\left(\mathrm{cm}^{3} \mathrm{sec}^{-1}\right)^{2}\left(\mathrm{gcm}^{-3}\right)\left(\mathrm{cm}^{-4} \mathrm{~cm}^{-1}\right) & \\
& =\mathrm{gcm}^{-2} \mathrm{sec}^{-2} \\
& =\text { Force/Volume }
\end{aligned}
$$

4.32 Let $\mathrm{n}_{\mathrm{v}}$, the viscous hydraulic force per unit element of fluid control volume, be defined as:

$$
\mathrm{n}_{\mathrm{v}}=\frac{\delta \mu_{\mathrm{s}} \eta}{\mathrm{d}_{\mathrm{c}}^{2}}
$$

4.33 Similarly, in a dimensional analysis using the cgs convention, we demonstrate the dimensional integrity of $\mathrm{n}_{\mathrm{v}}$, thusly:

$$
\begin{aligned}
\mathrm{n}_{\mathrm{v}}=\left(\mathrm{cm}^{3} \mathrm{sec}^{-1}\right)\left(\mathrm{gcm}^{-1} \mathrm{sec}^{-1}\right) & \left(\mathrm{cm}^{-2} \mathrm{~cm}^{-2}\right) \\
& =\mathrm{gcm}^{-2} \mathrm{sec}^{-2} \\
& =\text { Force/Volume }
\end{aligned}
$$

4.34 Finally, let us define BLT, the fluid wall boundary layer thickness, as follows:

$$
\mathrm{BLT}=\frac{\beta \mathrm{d}_{\mathrm{c}}}{2 \tau}
$$




\section{G. Quinn's Law}

5.10 We may now restate equation (40) as:

$$
\mathrm{P}_{\mathrm{Q}}=\frac{4 \pi \mathrm{r}_{h}}{3}+\frac{\delta \lambda \mathrm{n}_{k}}{2 \pi \mathrm{r}_{\mathrm{h}} \mathrm{n}_{\mathrm{v}}}
$$

5.11 Let us define the dimensionless drag normalized pressure gradient as:

$$
\frac{\Delta \mathrm{P}}{\mathrm{r}_{\mathrm{h}} \mathrm{n}_{\mathrm{v}} \mathrm{L}}=\mathrm{P}_{\mathrm{Q}}
$$

Where, $\Delta \mathrm{P} / \mathrm{L}=$ the pressure gradient; $\Delta \mathrm{P} /\left(\mathrm{r}_{\mathrm{h}} \mathrm{L}\right)=$ drag normalized pressure gradient. $\Delta \mathrm{P} /\left(\mathrm{r}_{\mathrm{h}} \mathrm{n}_{\mathrm{v}} \mathrm{L}\right)=$ drag normalized viscous friction factor.

Then, substituting for $\mathrm{P}_{\mathrm{Q}}$ in Equation (59), we may write:

$$
\underset{\mathrm{r}_{\mathrm{h}} \mathrm{n}_{\mathrm{v}} \mathrm{L}}{\Delta \mathrm{P}}=\frac{4 \pi \mathrm{r}_{h}^{2}}{3}+\frac{\delta \lambda \mathrm{n}_{k}}{2 \pi \mathrm{r}_{\mathrm{h}} \mathrm{n}_{\mathrm{v}}}
$$

Multiplying across by $\mathrm{n}_{\mathrm{v}}$ in Equation (61) gives:

$$
\frac{\Delta \mathrm{P}}{\mathrm{r}_{\mathrm{h}} \mathrm{L}}=\frac{4 \pi \mathrm{r}_{\mathrm{h}}^{2} \mathrm{n}_{\mathrm{v}}}{3}+\frac{\delta \lambda \mathrm{n}_{\mathrm{k}}}{2 \pi \mathrm{r}_{\mathrm{h}}}
$$

We can describe Equation (62) as:

drag normalized pressure gradient $=($ viscous term $)+($ kinetic term $)$

Equation (62) represents the instantaneous balanced equation with respect to the distribution of forces between the contributions of viscous and kinetic considerations. Unfortunately, however, it is not a useful equation from an empirical perspective, because in practice we measure $\Delta \mathrm{P}$, not $\left(\Delta \mathrm{P} / \mathrm{r}_{\mathrm{h}}\right)$.

Accordingly, multiplying across by $\mathrm{r}_{\mathrm{h}}$ in Equation (62), gives:

$$
\frac{\Delta \mathrm{P}}{\mathrm{L}}=\frac{4 \pi \mathrm{r}_{\mathrm{h}}{ }^{3} \underline{\mathrm{n}}_{\mathrm{v}}}{3}+\frac{\delta \lambda \mathrm{n}_{\mathrm{k}}}{2 \pi}
$$


Substituting for $\mathrm{n}_{\mathrm{v}}$ [Eq. (57)] and $\mathrm{n}_{\mathrm{k}}$ [Eq. (55)] in Equation (63), gives:

$$
\frac{\Delta \mathrm{P}}{\mathrm{L}}=\frac{4 \pi \mathrm{r}_{\mathrm{h}}^{3} \delta}{3 \mathrm{~d}_{\mathrm{c}}{ }^{2}} \underline{\mathrm{s}} \underline{\eta}+\frac{\delta^{2} \lambda \mu_{\mathrm{s}}^{2} \rho_{\mathrm{f}}}{\mathrm{d}_{\mathrm{c}}}
$$

Therefore, empirically, Equation (64) is the most useful equation for any practitioner. It demonstrates that when the fluid velocity, $\mu_{\mathrm{s}}$, tends to zero, (fluid at rest), and, therefore, the kinetic term ( $2^{\text {nd }}$ term on right hand side of Equation (64)) is negligible, the control volume element coefficient is represented by $4 \pi \mathrm{r}_{\mathrm{h}}{ }^{3} / 3=256 \pi / 3=268$ approx., since this is the multiplier in the viscous term of Equation (64) corresponding to the pressure gradient $\Delta \mathrm{P} / \mathrm{L}$. In other words, the entire control volume element is assigned to viscous considerations only, and is represented by the volume of a sphere having a radius $r_{h}$, the coefficient of fluid drag. We can show this algebraically, as follows (neglecting the kinetic term in Equation (63)):

$$
\frac{\Delta \mathrm{P}}{\mathrm{L}}=\frac{4 \pi \mathrm{rr}_{\mathrm{h}}^{3} \mathrm{n}_{\mathrm{v}}}{3}
$$

5.12 Equation (65) represents the empirically meaningful relationship between the measured pressure gradient (left hand side), and the fluid motion term (right hand side) when the fluid flow rate is very close to zero, i.e., kinetic contributions are negligible. Note that in this fluid flow regime, the fluid motion term is the product of two entities: $\mathrm{n}_{\mathrm{v}}=$ the viscous hydraulic force exerted per unit element of fluid control volume and $\left(4 \pi \mathrm{r}_{\mathrm{h}}{ }^{3} / 3=268\right) \mathrm{a} / \mathrm{k} / \mathrm{a}$ the "viscous constant".

[Incidentally, we point out that the value of 270 for the viscous constant in the Kozeny/Blake equation was identified as early as 1965 by J.C. Giddings [26, 27.].

5.13 We can now appreciate that the distribution of forces between viscous and kinetic contributions is captured by maintaining the drag-normalized value of the viscous fluid control element normalization coefficient, $\mathrm{k}_{1}=64 \pi / 3$ and the drag-normalized value of the kinetic fluid control element normalization coefficient, $\mathrm{k}_{2}=1 /(8 \pi)$. Thus, the constant $\mathrm{k}_{1}$ acts as a dimensionless normalization coefficient for the hydraulic viscous forces, on the one hand, and $\mathrm{k}_{2}$ acts as a dimensionless normalization coefficient for the hydraulic kinetic forces, on the other hand. In addition, note that in the viscous term, this normalization coefficient is directly proportional to the second power of the drag coefficient, whereas in the kinetic term, it is inversely proportional to the first power of the drag coefficient, which is exactly what we intuitively observe in the world around us. In other words, our experience tells us that as the surface area in contact with the flowing fluid increases, the pressure gradient increases (direct proportionality); but as the diameter of a conduit increases, the pressure gradient decreases (indirect proportionality).

5.14 We digress at this point to mention an aspect of our theoretical model which contradicts a common feature of conventional wisdom. That feature is the notion that the Reynolds number, $\mathrm{R}_{\mathrm{e}}$, is the ratio of kinetic to viscous forces. Correctly stated, the modified Reynolds number, $\mathrm{R}_{\mathrm{em}}$, 
is the ratio of the kinetic forces per unit control volume element to the viscous forces per unit control volume element, which involves the ratio of $\mathrm{k}_{1} / \mathrm{k}_{2}$, a discrepancy factor of approximately 1,686 .

The conventional Reynolds number concept, $R_{e}$, was derived by Sir Osborne Reynolds (1883) in experiments based upon an "idealized" fluid flow channel, the wall roughness and boundary layer of which he did not take into account. His idealized channel, therefore, would be represented by values for $\delta=1$ and $\lambda=1$, in our model, i.e., no wall effect of any kind and an undefined kinetic component of driving force, $\Delta \mathrm{P}$, since the value of $\delta=1$ represents a point of discontinuity in the Q porosity function, $\varepsilon$. Accordingly, one could argue that the concept of the conventional Reynolds number is an undefined parameter in the real world, which might explain many of the anomalies in conventional wisdom, not the least of which is the inability to provide an analytical solution to the Navier-Stokes equation.

5.15 Now that we have properly balanced the pressure flow equation and assigned the correct variables to each of its compartments with respect to the contributions of viscous and kinetic considerations, and, in addition, have quantified the pressure gradient by setting the correct magnitude of the control volume element of fluid, we may proceed to gather terms and evaluate the various physical phenomena occurring within the flowing fluid channel.

5.16 Let us now define the wall-adjusted fluid current as:

$$
\lambda Q_{\mathrm{n}}=\mathrm{C}_{\mathrm{Q}}
$$

Where, $\mathrm{C}_{\mathrm{Q}}=$ the dimensionless wall-adjusted fluid current.

It follows that we may now write:

$$
\beta=\frac{\mathrm{k}_{1}}{\left(\omega \mathrm{Q}_{\mathrm{N}}+\mathrm{k}_{1}\right)}
$$

Where, $\beta=$ the instantaneous fluid/wall boundary.

5.17 It also follows that we may now rewrite Equation (40) as:

$$
\mathrm{P}_{\mathrm{Q}}=\frac{64 \pi}{3}+\frac{\mathrm{C}_{\mathrm{Q}}}{8 \pi}
$$

We hereby designate Equation (68) as Quinn's Law of Fluid Dynamics. It states that all pressure-driven flow in closed conduits can be represented by a linear dimensionless relationship, having a constant slope of $1 /(8 \pi)$ and an intercept of $64 \pi / 3$. This relationship is shown in Fig.7. 


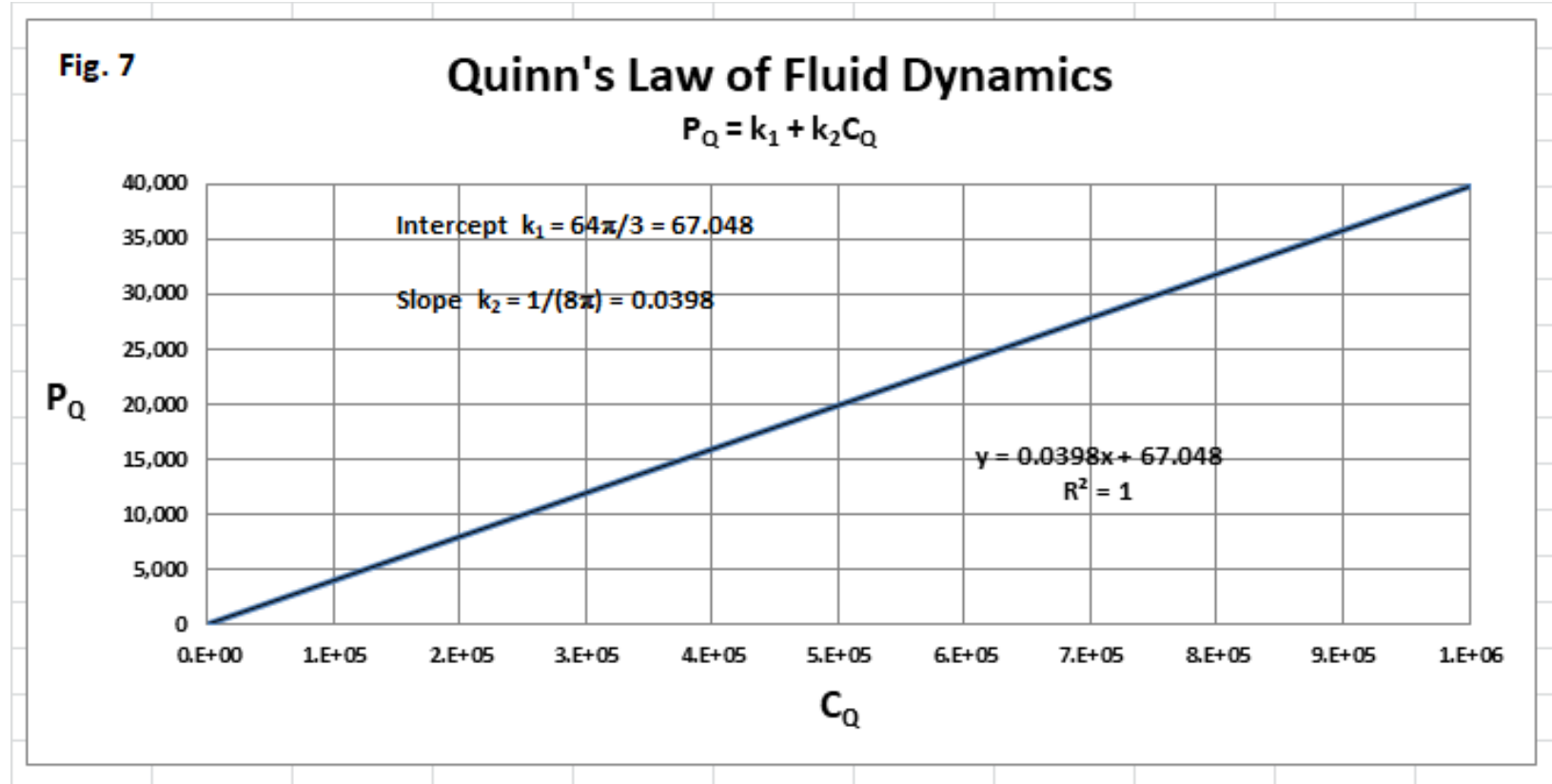

5.18 If we replace the velocity term in Equation (64) by a flow rate term and substitute $\mathrm{r}_{\mathrm{h}}=4$, we get:

$$
\frac{\Delta \mathrm{P}}{\mathrm{L}}=\frac{1024 \delta \mathrm{q \eta}}{3 \mathrm{D}^{2} \mathrm{~d}_{\mathrm{c}}^{2}}+\frac{8 \delta^{2} \lambda \mathrm{q}^{2} \rho_{\mathrm{f}}}{\pi^{3} \mathrm{D}^{4} \mathrm{~d}_{\mathrm{c}}}
$$

In the special case of an empty conduit, where $\delta=1 / 8, \mathrm{~d}_{\mathrm{c}}=\mathrm{D}$ and $\pi=22 / 7$, it follows that we may write:

$$
\frac{\Delta P}{L}=\frac{128 q \eta}{3 D^{4}}+\frac{\lambda q^{2} \rho_{f}}{248 D^{5}}
$$

Accordingly, we can conclude that in the case of an empty conduit, the pressure gradient is:

a. inversely proportional to the fourth power of the conduit diameter in the viscous term, and

b. inversely proportional to the fifth power of the conduit diameter in the kinetic term.

Hence the empirical importance of measuring accurately the conduit diameter in experiments related to conduit permeability.

Finally, we point out that the viscous term in Equation (70) corresponds to Poiseuille's equation for laminar flow. Note, however, that by comparison, Poiseuille's equation has the coefficient of $\pi$ in the denominator, rather than the coefficient of 3 in Equation (70). Therefore, Poiseuille's equation understates the pressure gradient by 1 part in 22, a discrepancy of $4.55 \%$, which falls within the measurement error of many typical engineering applications. 
5.19 Let us now consider a quadratic relationship for the volumetric fluid flow, q, by expressing Equation (69) above, as follows:

$$
\mathrm{aq}^{2}+\mathrm{bq}+\mathrm{c}=0
$$

Where, $\mathrm{b}=\mathrm{a}$ variable coefficient of $\mathrm{q} ; \mathrm{a}=\mathrm{a}$ variable coefficient of $\mathrm{q}^{2}$; and $\mathrm{c}=\mathrm{a}$ constant .

Rearranging Equation (71) gives:

$$
\mathrm{aq}^{2}+\mathrm{bq}=-\mathrm{c}
$$

Substituting $\Delta \mathrm{P}$ for $-\mathrm{c}$ in Equation (72) and rearranging gives:

$$
\Delta \mathrm{P}=\mathrm{aq}^{2}+\mathrm{bq}
$$

Solving Equation (73) for q gives:

$$
q=\left[-b \pm v\left(b^{2}+4 a \Delta P\right)\right]
$$

5.20 Let us define the coefficients $a$ and $b$ as follows:

$$
\begin{aligned}
& a=\frac{8 \delta^{2} \lambda \rho_{f} L}{\left(\pi^{3} D^{4} d_{c}\right)} \\
& b=\frac{1024 \delta \eta L}{\left(3 D^{2} d_{c}^{2}\right)}
\end{aligned}
$$

Substituting for a and b in Equation (73), we get Eq. (69):

$$
\frac{\Delta \mathrm{P}}{\mathrm{L}}=\frac{1024 \delta \eta \mathrm{q}}{\left(3 \mathrm{D}^{2} \mathrm{~d}_{\mathrm{c}}{ }^{2}\right)}+\frac{8 \delta^{2} \lambda \rho_{\mathrm{f}} \mathrm{q}^{2}}{\left(\pi^{3} \mathrm{D}^{4} \mathrm{~d}_{\mathrm{c}}\right)}
$$

5.21 In order to cross-reference with the conventional literature for packed conduits, we may now substitute for HQC variables as follows: 
Substituting for $\mathrm{d}_{\mathrm{c}}$ [Eq. (24)] and $\delta$ [Eq.(41)] in Equation (69), and rearranging gives:

$$
\frac{\Delta \mathrm{P}}{\mathrm{L}}=\frac{1024\left(1-\varepsilon_{0}\right)^{2} \eta \mathrm{q}}{\left(3 \mathrm{D}^{2} \varepsilon_{0}^{3} \mathrm{~d}_{\mathrm{p}}{ }^{2}\right)}+\frac{8\left(1-\varepsilon_{0}\right) \lambda \rho_{f}}{\left(\pi^{3} \mathrm{D}^{4} \varepsilon_{0}{ }^{6} d_{p}\right)}
$$

Switching the fluid flow parameter from volumetric flow rate to superficial velocity, we may now write:

$$
\frac{\Delta \mathrm{P}}{\mathrm{L}}=\frac{256 \pi\left(1-\varepsilon_{0}\right)^{2} \mu_{\mathrm{s}} \eta}{\left(3 \varepsilon_{0}{ }^{3} \mathrm{~d}_{\mathrm{p}}{ }^{2}\right)}+\frac{\left(1-\varepsilon_{0}\right) \lambda \rho_{\mathrm{f}} \mu_{\mathrm{s}}{ }^{2}}{\left(2 \pi \varepsilon_{0} \mathrm{~d}_{\mathrm{p}}\right)}
$$

Substituting for $256 \pi / 3=A$, on the left hand side of Equation $(78)$, and $\lambda /\left(2 \pi \varepsilon_{0}{ }^{3}\right)=B$, on the right hand side of Equation (78), gives:

$$
\left.\frac{\Delta \mathrm{P}}{\mathrm{L}}=\frac{\mathrm{A}\left(1-\varepsilon_{0}\right)^{2} \mu_{\mathrm{s}} \eta}{\left(\varepsilon_{0}^{3} \mathrm{~d}_{\mathrm{p}}{ }^{2}\right)}+\frac{\mathrm{B}\left(1-\varepsilon_{0}\right) \rho_{\mathrm{f}}}{\left(\varepsilon_{0} \mathrm{\mu}_{\mathrm{p}}^{2}\right.} \mathrm{d}_{\mathrm{p}}\right)
$$

We point out that Equation (79) corresponds to the general format of the Ergun equation. However, we call it the Q-modified Ergun equation because the "constants" of $A=268$ (approx.) and $B=\lambda /\left(2 \pi \varepsilon_{0}{ }^{3}\right)$, have been modified from Ergun's original constant values of 150 and 1.75 for $A$ and $B$, respectively, and most importantly, $B$ is not a constant, but rather is a function of both $\lambda$ and $\varepsilon 0$.

\section{H. Modeling the HQC as a Harmonic Oscillator}

\section{a. The Spatial Coordinates}

We shall now return to the motion of the fluid within the HQC and describe it in terms of its instantaneous velocity coordinates in three dimensions, i.e., $\mathrm{x}, \mathrm{y}, \mathrm{z}$..

6.10 Thus, we may write:

$$
\mathrm{v}=\mathrm{v}_{\mathrm{x}}+\mathrm{v}_{\mathrm{y}}+\mathrm{v}_{\mathrm{z}}
$$

where, $v=$ the total instantaneous velocity.

6.11 Let us define the conduit frictional time interval as follows:

$$
\mathrm{t}_{0}=\frac{\pi \mathrm{D}^{2} \mathrm{~L} \varepsilon_{\mathrm{t}}}{4 \mathrm{q}}
$$


Where, $\mathrm{t}_{0}=$ time to displace one conduit volume of fluid.

6.12 Let us define the wall shear stress as follows:

$$
\tau_{\mathrm{w}}=\frac{\Delta \mathrm{PD}}{4 \mathrm{~L}}
$$

Where, $\tau_{\mathrm{w}}=$ the wall shear stress.

6.13 Let us define the frictional fluid velocity as follows:

$$
\mu_{\mathrm{f}}=\sqrt{\left(\tau_{\mathrm{w}} / \rho_{\mathrm{f}}\right)}
$$

Where, $\mu_{\mathrm{f}}=$ the frictional fluid velocity.

6.14 Let us define the period of the fluid motion as follows:

$$
\mathrm{T}=\frac{2 \pi}{\omega}
$$

Where, $\mathrm{T}=$ the period of the fluid motion.

6.15 Let us define the frequency of the fluid motion as follows:

$$
\phi=\frac{1}{\mathrm{~T}}
$$

Where, $\phi=$ the frequency of the fluid motion.

6.16 Let us define the maximum displacement amplitude of the fluid motion as follows:

$$
\mathrm{M}_{0}=\frac{\mathrm{d}_{\mathrm{c}}}{2}
$$

Where, $\mathbf{M}_{0}=$ the maximum displacement amplitude of the fluid motion (scale factor).

6.17 Let us define the instantaneous displacement amplitude as follows: 


$$
M=M_{0} \exp ^{(-\omega t 0)}
$$

Where, $\mathrm{M}=$ the instantaneous displacement amplitude of the fluid motion.

Note that the negative sign in the exponent in equation (87) represents the fact that the conduit wall frictional force acts in the opposite direction to the fluid motion and, hence, the motion is "damped" by wall friction.

6.18 Let us define the instantaneous displacement amplitude in the x-axis plane as follows:

$$
\mathrm{x}=\mathrm{M} \cos \mathrm{P}_{\mathrm{Q}}
$$

Where, $\mathrm{x}=$ the instantaneous fluid displacement amplitude in the $\mathrm{x}$-axis plane.

6.19 Let us define the instantaneous fluid velocity in the $\mathrm{x}$-axis plane as follows:

$$
\mathrm{v}_{\mathrm{x}}=-\frac{\mathrm{M} \lambda \sin \mathrm{P}_{\mathrm{Q}}}{\phi_{\mathrm{h}}}
$$

Where, $\mathrm{v}_{\mathrm{x}}=$ the instantaneous fluid velocity in the $\mathrm{x}$-axis plane.

6.20 Let us define the instantaneous fluid acceleration in the $\mathrm{x}$-axis plane as follows:

$$
\mathrm{f}_{\mathrm{x}}=-\frac{\mathrm{M} \lambda^{2} \cos \mathrm{P}_{\mathrm{Q}}}{\phi_{\mathrm{h}}{ }^{2}}
$$

Where, $f_{x}=$ the instantaneous fluid acceleration in the $\mathrm{x}$-axis plane.

6.21 Let us define the instantaneous motion displacement in the y-axis plane as follows:

$$
\mathrm{y}=\mathrm{M} \sin \mathrm{P}_{\mathrm{Q}}
$$

Where, $y=$ the instantaneous fluid displacement in the $y$-axis plane.

6.22 Let us define the instantaneous fluid velocity in the y-axis plane as follows: 


$$
\mathrm{v}_{\mathrm{y}}=\frac{\mathrm{M} \lambda \cos \mathrm{P}_{\mathrm{Q}}}{\phi_{\mathrm{h}}}
$$

Where, $v_{y}=$ the instantaneous fluid velocity in the $y$-axis plane.

6.23 Let us define the instantaneous fluid acceleration in the y-axis plane as follows:

$$
\mathrm{f}_{\mathrm{y}}=\frac{-\mathrm{M} \lambda^{2} \sin \mathrm{P}}{\phi_{\mathrm{h}}^{2}}
$$

Where, $f_{y}=$ the instantaneous fluid acceleration in the $y$-axis plane.

6.24 Let us define the instantaneous motion displacement in the $\mathrm{z}$-axis plane as follows:

$$
\mathrm{z}=\mathrm{M} \cos \left(\pi / 4-\mathrm{P}_{\mathrm{Q}}\right)
$$

Where, $\mathrm{z}=$ the instantaneous fluid displacement in the $\mathrm{z}$-axis plane.

6.25 Let us define the instantaneous fluid velocity in the z-axis plane as follows:

$$
\mathrm{v}_{\mathrm{z}}=\frac{-\mathrm{M} \lambda \sin \left(\pi / 4-\mathrm{P}_{\mathrm{Q}}\right)}{\phi_{\mathrm{h}}}
$$

Where, $\mathrm{v}_{\mathrm{z}}=$ the instantaneous fluid velocity in the $\mathrm{z}$-axis plane.

6.26 Let us define the instantaneous fluid acceleration in the z-axis plane as follows:

$$
\mathrm{f}_{\mathrm{z}}=\frac{-\mathrm{M} \lambda^{2} \cos \left(\pi / 4-\mathrm{P}_{\mathrm{Q}}\right)}{\phi_{\mathrm{h}}^{2}}
$$

Where, $\mathrm{f}_{\mathrm{z}}=$ the instantaneous fluid acceleration in the $\mathrm{z}$-axis plane.

\section{b. The Hypothetical Q Unit Cell}

We shall now describe the dimensionless manifestation of the fluid motion in the HQC which we term the "Hypothetical Q Unit Cell" (HQUC).

6.27 Let us define the dimensionless instantaneous motion displacement in the $\mathrm{x}$-axis plane as follows: 


$$
\mathrm{x}^{*}=\frac{\mathrm{x}}{\mathrm{M}_{0}}
$$

Where, $\mathrm{x}^{*}=$ the dimensionless instantaneous fluid displacement in the $\mathrm{x}$-axis plane.

6.28 Let us define the dimensionless instantaneous fluid velocity in the $\mathrm{x}$-axis plane as follows:

$$
\mathrm{v}_{\mathrm{x}} *=\underline{\mathrm{v}_{\mathrm{x}}}
$$

Where, $\mathrm{v}_{\mathrm{x}} *=$ the dimensionless instantaneous fluid velocity in the $\mathrm{x}$-axis plane.

6.29 Let us define the dimensionless instantaneous motion displacement in the y-axis plane as follows:

$$
\mathrm{y}^{*}=\frac{\mathrm{y}}{\mathrm{M}_{0}}
$$

Where, $\mathrm{y}^{*}=$ the dimensionless instantaneous fluid displacement in the $\mathrm{y}$-axis plane.

6.30 Let us define the dimensionless instantaneous fluid velocity in the y-axis plane as follows:

$$
\mathrm{v}_{\mathrm{y}}{ }^{*}=\underline{\mathrm{v}}_{\mathrm{y}}
$$

Where, $\mathrm{v}_{\mathrm{y}}{ }^{*}=$ the dimensionless instantaneous fluid velocity in the $\mathrm{y}$-axis plane.

6.31Let us define the dimensionless instantaneous motion displacement in the z-axis plane as follows:

$$
\mathrm{z}^{*}=\frac{\mathrm{z}}{\mathrm{M}_{0}}
$$

Where, $\mathrm{z}^{*}=$ the dimensionless instantaneous fluid displacement in the $\mathrm{x}$-axis plane.

6.32 Let us define the dimensionless instantaneous fluid velocity in the z-axis plane as follows:

$$
\mathrm{v}_{\mathrm{z}} *=\frac{\mathrm{v}_{\mathrm{z}}}{\mu_{\mathrm{f}}}
$$


Where, $\mathrm{v}_{\mathrm{z}}^{*}=$ the dimensionless instantaneous fluid velocity in the $\mathrm{z}$-axis plane.

\section{Exploring the QFFM}

In order to further explore the implications of the HQC, we propose a series of worked examples which will articulate the role of the various parameters embodied in the QFFM. Our worked examples have the following common independent working variables: $\mathrm{D}=0.46 \mathrm{~cm} ; \mathrm{L}=10 \mathrm{~cm}$; $\Omega_{\mathrm{p}}=1.0 ; \mathrm{d}_{\mathrm{p}}=0.001 \mathrm{~cm} ; \eta=0.01$ poise$;$ and $\rho_{\mathrm{f}}=1.0 \mathrm{~g} / \mathrm{mL}$.

\section{a. The HQC Fluid Current Normalization Coefficient $\lambda$}

7.10 We begin with 8 worked examples involving an empty conduit having variable relative wall roughness $\left(\mathrm{k}_{\mathrm{dc}}\right)$ values, as shown in Fig. 7A and 7B. They are as follows:

1. Empty conduit-smooth wall $\left(\mathrm{k}_{\mathrm{dc}}=0\right)$

2. Empty conduit-roughened wall $\left(\mathrm{k}_{\mathrm{dc}}=0.0001\right)$

3. Empty conduit-roughened wall $\left(\mathrm{k}_{\mathrm{dc}}=0.001\right)$

4. Empty conduit-roughened wall $\left(\mathrm{k}_{\mathrm{dc}}=0.01\right)$

5. Empty conduit-roughened wall $\left(\mathrm{k}_{\mathrm{dc}}=0.03\right)$

6. Empty conduit-roughened wall $\left(\mathrm{k}_{\mathrm{dc}}=0.05\right)$

7. Empty conduit-roughened wall $\left(\mathrm{k}_{\mathrm{dc}}=0.1\right)$

8. Empty conduit-roughened wall $\left(\mathrm{k}_{\mathrm{dc}}=0.5\right)$

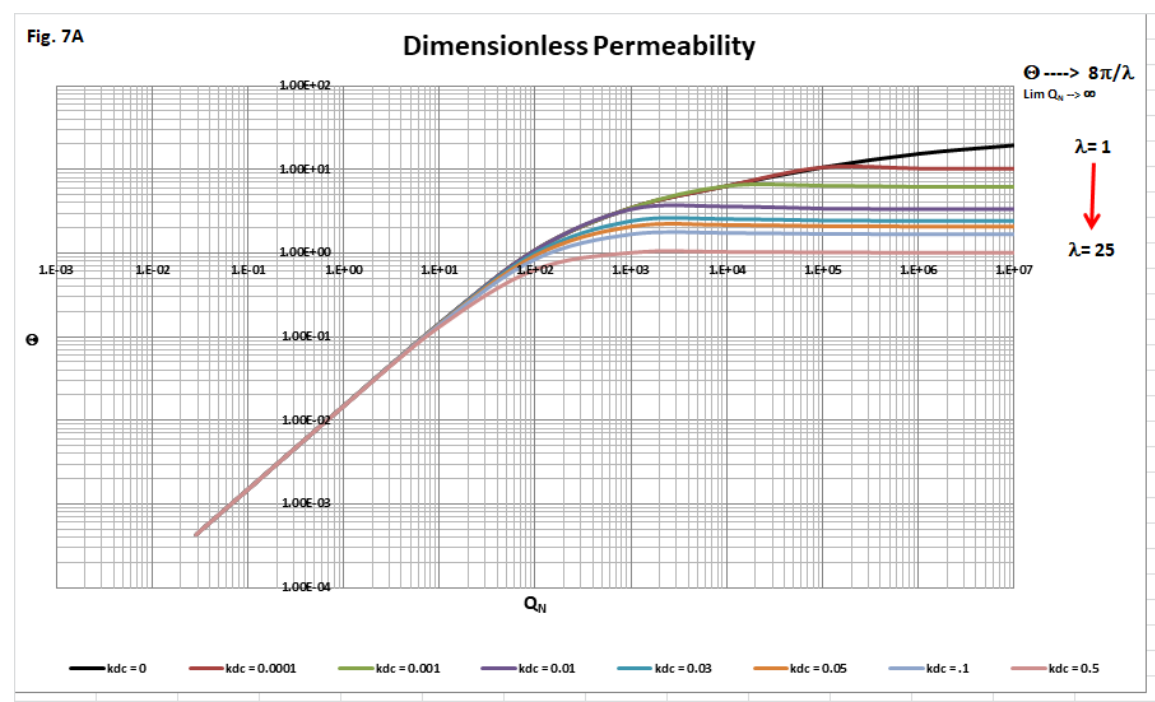




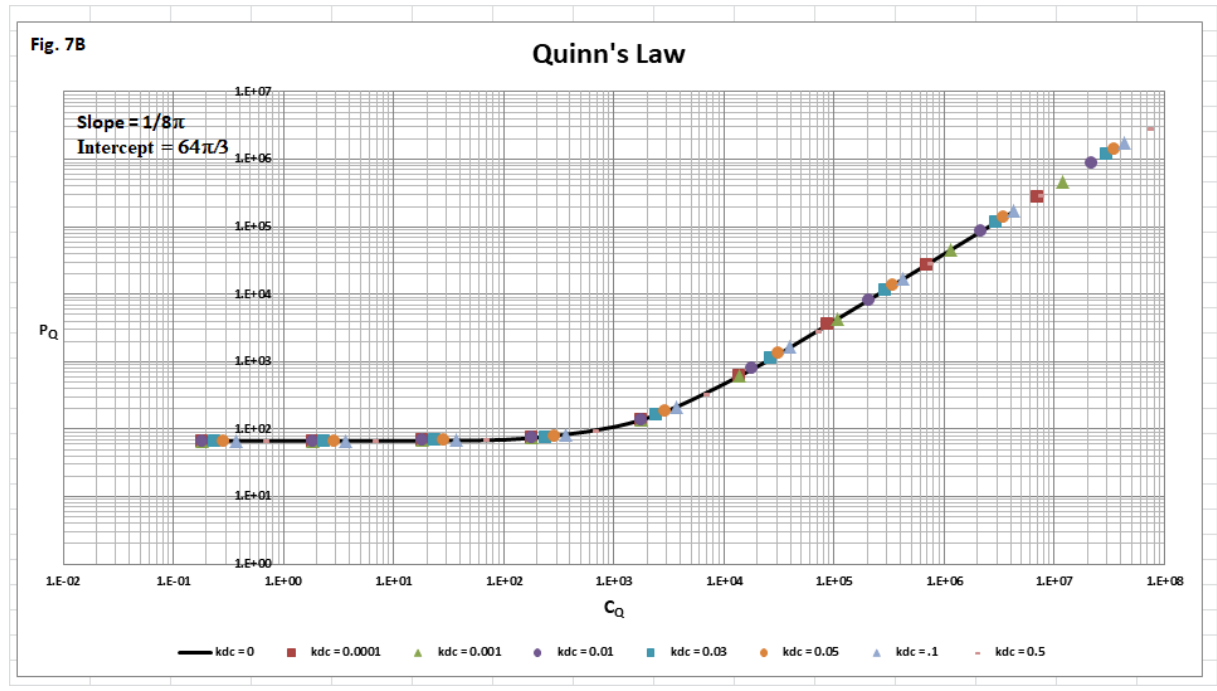

As shown in Fig. 7A on a log-log plot of dimensionless permeability, $\Theta$, versus fluid current, $Q_{N}$, the 8 worked examples produce characteristic dimensionless permeability line shapes, which vary according to the value of $\lambda$ for each example, at high values of $Q_{N}$. There is no differentiation between these worked examples at low values of $Q_{N}$, because the wall roughness is buried beneath the wall boundary layer. Larger values of $\lambda$ produce lower dimensionless permeability at high values of $Q_{N}$ because the residual secondary wall effect is commensurate with the wall roughness coefficient.

On the other hand, as shown in Fig. 7B, also in a $\log -\log$ format of $\mathrm{P}_{\mathrm{Q}}$ versus $\mathrm{C}_{\mathrm{Q}}$, our typical graphical representation of Quinn's Law for the same examples, the data yields a straight line whose intercept and slope are represented by the constants $\mathrm{k}_{1}=64 \pi / 3$ and $\mathrm{k}_{2}=1 / 8 \pi$, respectively. Note that the exact location for each of the 8 examples on the $\mathrm{x}$-axis of the plot is determined by the unique value of $\lambda$ at each measured flow rate. We show the axis of this plot in a log-log format to highlight the relationship at the extremes of the values for wall-adjusted fluid current $\mathrm{C}_{\mathrm{Q}}$. The location of any given data point on the $\mathrm{x}$ axis for each worked example moves to higher values as a function of wall roughness, at comparable flow rates.

\section{b. The HQC Tortuosity Coefficient $\tau$}

7.11 In our model, a packed conduit is the general case of the theory and an empty conduit a special case. They are differentiated in the theory, however, by virtue of the HQC tortuosity coefficient $\tau$. This distinction is driven by the fact that $\tau=\delta \gamma$, is a constant less than unity for an empty conduit (0.188) but is greater than unity and is not a constant for a packed conduit. In fact, for most practical packed conduits, the tortuosity factor is enormous by comparison to that of an empty conduit. We show a comparison in Fig. 8. 


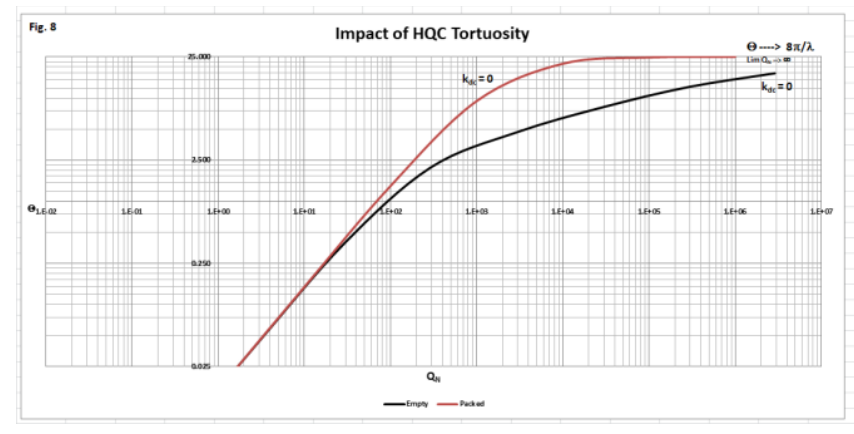

HQC tortuosity manifests itself in the normalized boundary layer parameter, which we designate as the primary wall effect $W_{1}$, and is best viewed on a plot of dimensionless permeability, $\Theta$, versus fluid current, $Q_{N}$.

As shown in the plot, on the one hand, the normalized boundary layer in the packed conduit is infinitesimally thin, by virtue of the enormous tortuosity value, $\tau=1.6 \times 10^{9}$, which means that the primary wall effect is negligible and, because the wall roughness is also negligible when $\mathrm{k}=$ 0 , there is no net wall effect. Consequently, for the packed conduit, the value of $\lambda$ is close to the value of 1 for all values of $Q_{N}$, which results in the higher permeability line shown in the plot.

On the other hand, in the case of an empty conduit, in which the tortuosity value is extremely low, $\tau=0.188$, the boundary layer is wider at low values of $Q_{N}$ and becomes thinner as the value of $Q_{N}$ increases. This results in a changing value of $\lambda$ as a function of $Q_{N}$, which, in turn, produces the lower permeability line shown in the plot. At very large values of $Q_{N}$, however, the boundary layer is essentially completely dissipated $(\lambda=1)$, in which case the dimensionless permeability of an empty conduit is identical to that of a packed conduit, when the HQC is hydraulically smooth $\left(\mathrm{k}_{\mathrm{dc}}=0\right)$, and it approaches the upper limit value of $8 \pi$ in the limit as $\mathrm{Q}_{\mathrm{N}}$ tends to infinity.

Thus, the dimensionless permeabilities of an empty and packed conduit are different for a hydraulically smooth surface at low values of $Q_{N}$, but are identical in the limit as $Q_{N}$ tends to infinity.

7.12 In addition, the value of $\lambda$ also affects the time constant $T$, as shown in Fig. 8A, in the case of a packed conduit. 


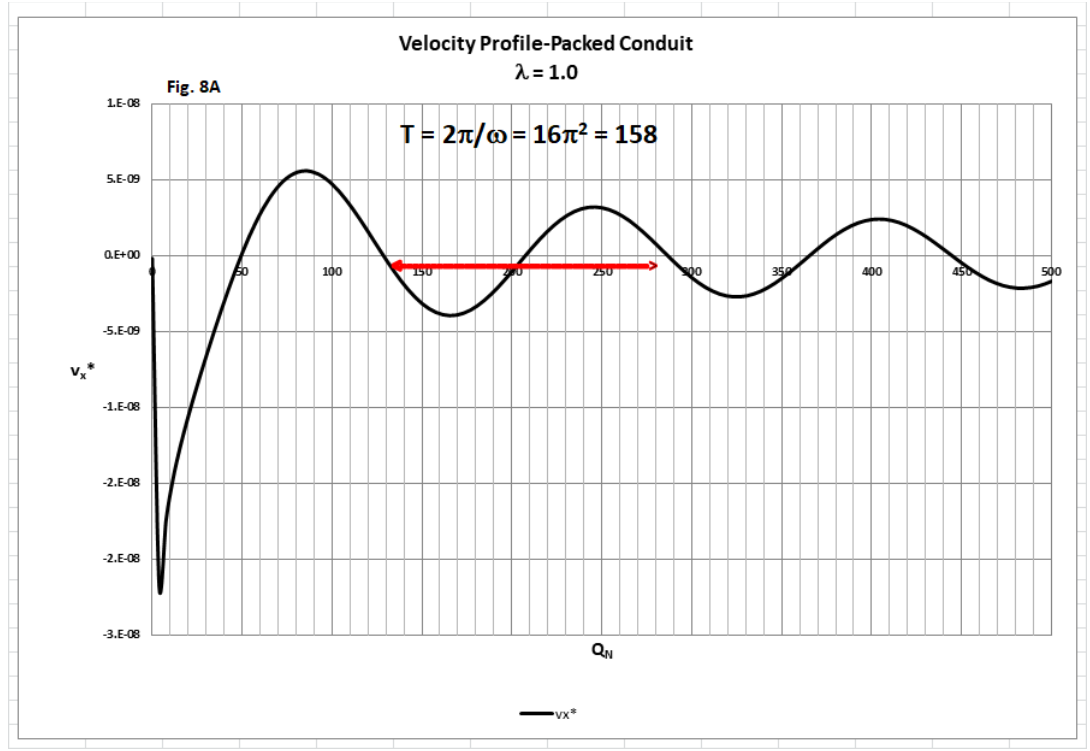

As shown in Fig. 8A, the time constant $\mathrm{T}$ is represented by one full rotation of the fluid, which is shown on the $\mathrm{x}$ axis. In the case of a packed conduit, the value of $\mathrm{T}=158$. This represents the maximum time constant possible since it represents a $\lambda$ value of unity, which, in turn, represents the minimum theoretical value of $\lambda$.

On the other hand, in the case of an empty conduit in which the value of $\lambda$ maybe greater than 1 , the value of the time constant $\mathrm{T}$ will always be less than that for a packed conduit, as shown in Fig. 8B.

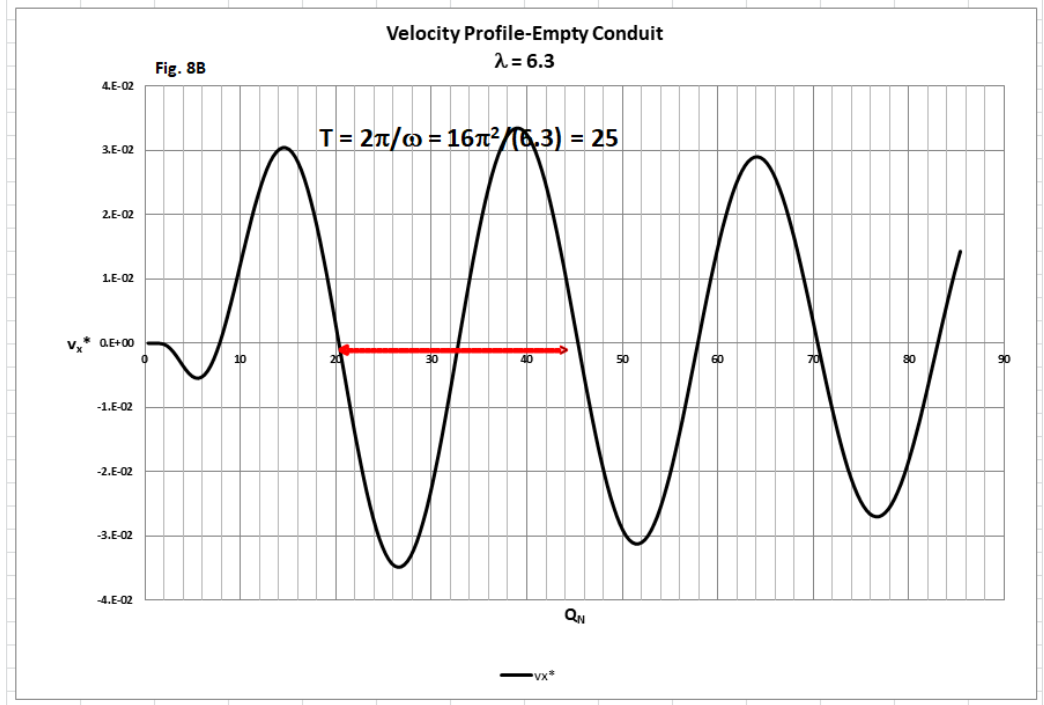

As shown in the plot for an empty hydraulically smooth conduit, when the value of $\lambda=6.3$, the value of $T=25$. Of course, the value of $\lambda$ will change at higher values of $Q_{N}$, as the boundary layer dissipates, which produces a corresponding increase in the time constant. 
As is also apparent in Figs. 8A and 8B, the fluid motion is "damped" in two ways; firstly, by wall friction, which is apparent at low values of $\mathrm{Q}_{\mathrm{N}}$, when wall friction has its major impact and, secondly, by fluid friction, which becomes increasingly dominant with higher values of $\mathrm{Q}_{\mathrm{N}}$.

\section{c. The HQC net wall effect $W_{N}$}

7.13 In our theoretical model, we begin with a description of the wall effect by focusing on our definition for the dimensionless boundary layer, $\beta_{0}$. As shown in Fig. 9A, the boundary layer varies from a value of unity at low values of $Q_{N}$ to a value approaching zero at high values of $Q_{N}$.

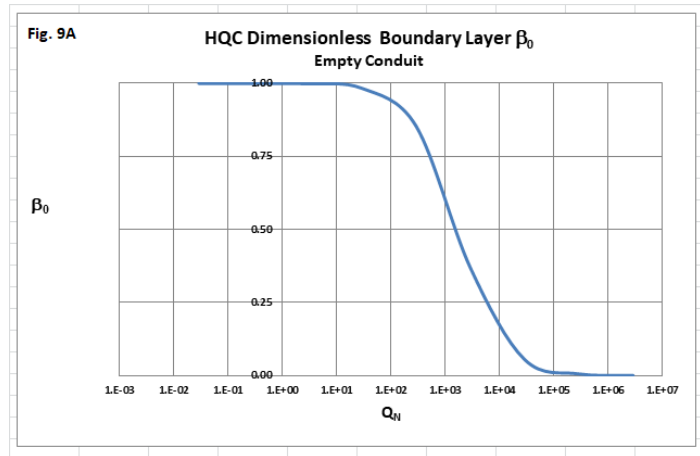

The characteristic shape in this plot underlies the fluid current changes from having fluid current-velocity streamlines parallel to the walls of the channel (laminar flow), at low values of $\mathrm{Q}_{\mathrm{N}}$, to those which are more concentric with the center point of the channel, at high values of $\mathrm{Q}_{\mathrm{N}}$ (turbulent flow). This change in the fluid flow profile characteristics from parabolic in nature to more circular in nature disrupts the boundary layer, which, in turn, becomes progressively thinner as the value of $Q_{N}$ increases. It is the value of the fluid current, $Q_{N}$, which produces the rotational component in the fluid current. More rotation at higher values of $\mathrm{Q}_{\mathrm{N}}$ reduces the thickness of the boundary layer.

7.14 Next we consider the HQC primary wall effect, $\mathrm{W}_{1}$, shown in Fig. 9B.

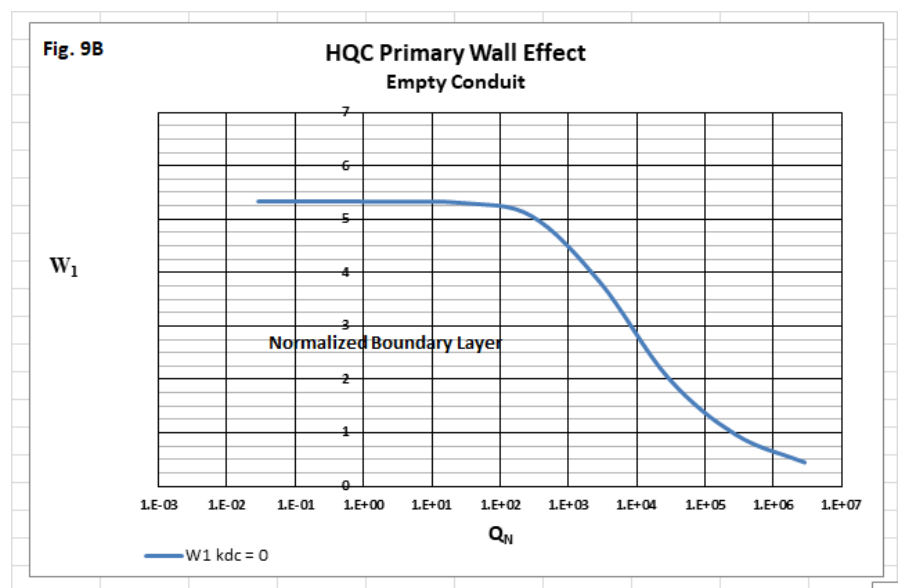

As shown in the plot, the primary wall effect is the boundary layer normalized for fluid channel tortuosity. Accordingly, in the case of an empty conduit (shown here) in which the tortuosity coefficient is less than unity, the primary wall effect is enhanced. For instance, in the example 
shown in the plot for an empty conduit, the normalized boundary layer is increased from unity, for the un-normalized boundary layer, to approximately 5.5. In contrast to an empty conduit, however, the primary wall effect in the case of a packed conduit is virtually negligible because the tortuosity coefficient is typically very, very large.

7.15 We now proceed to evaluate the HQC secondary wall effect, $\mathrm{W}_{2}$, as shown in Fig. 9C.

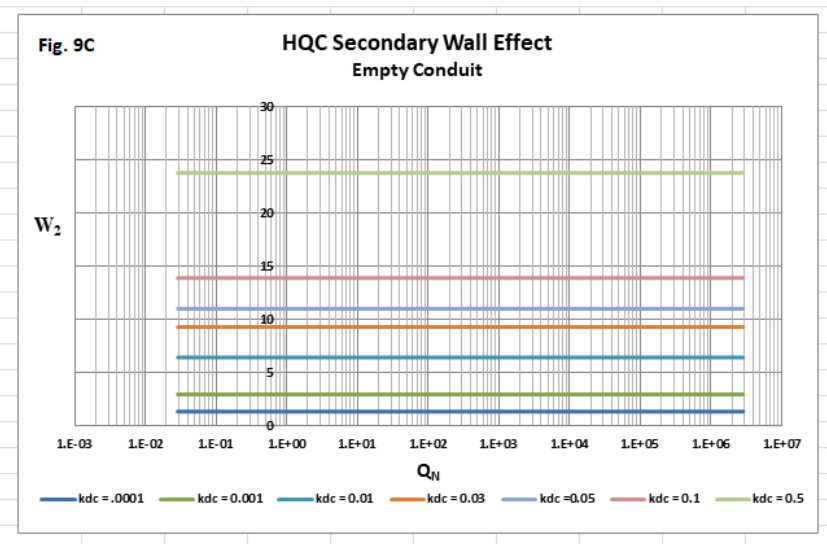

As shown in the plot, the secondary HQC wall effect, $\mathrm{W}_{2}$, is simply the degree of relative roughness on the inner channel wall. It increases as the relative roughness coefficient increases and is constant for a given value of the wall relative roughness coefficient, $\mathrm{k}_{\mathrm{dc}}$, throughout the entire fluid flow regime from low to high values of $Q_{N}$.

7.16 Next we identify the residual secondary wall effect, $\mathrm{Q}_{2 \mathrm{R}}$, which is the degree to which the relative roughness coefficient "punches through" the normalized boundary layer. This is shown in Fig. 9D.

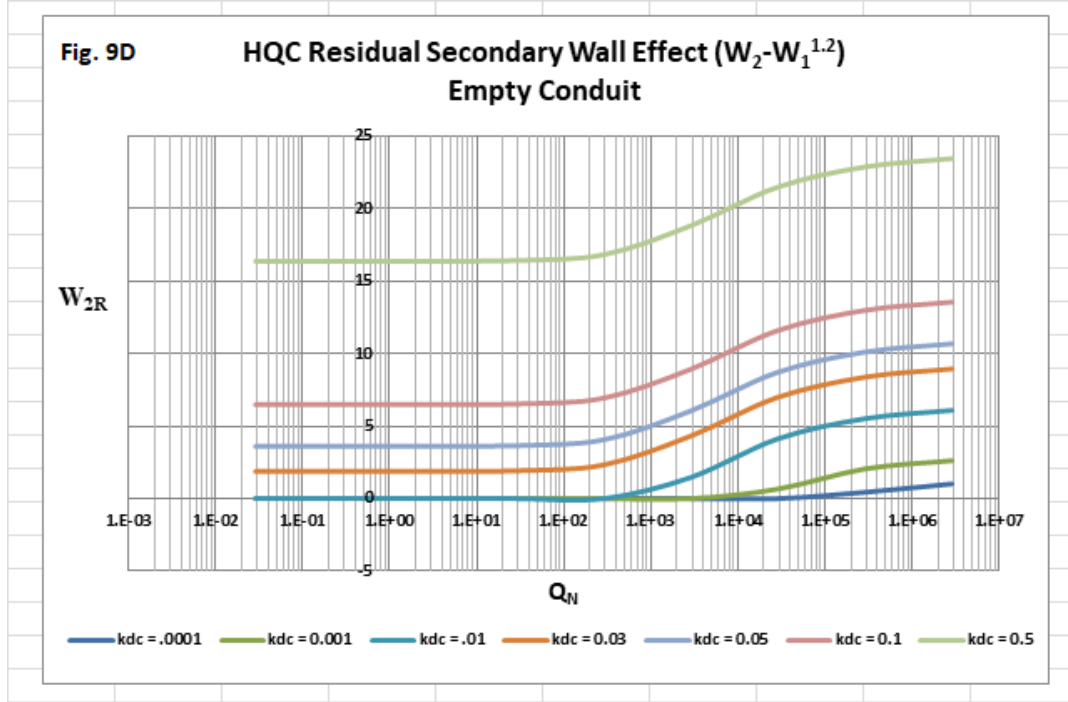


As shown in the plot, the secondary wall effect is masked by the normalized boundary layer at low values of $Q_{N}$ where the boundary layer is thickest, and gradually becomes more dominant, as it protrudes beyond the boundary layer at higher values of $Q_{N}$.

7.17 Next we evaluate the HQC net wall effect, $W_{N}$, as shown in Fig. 9E.

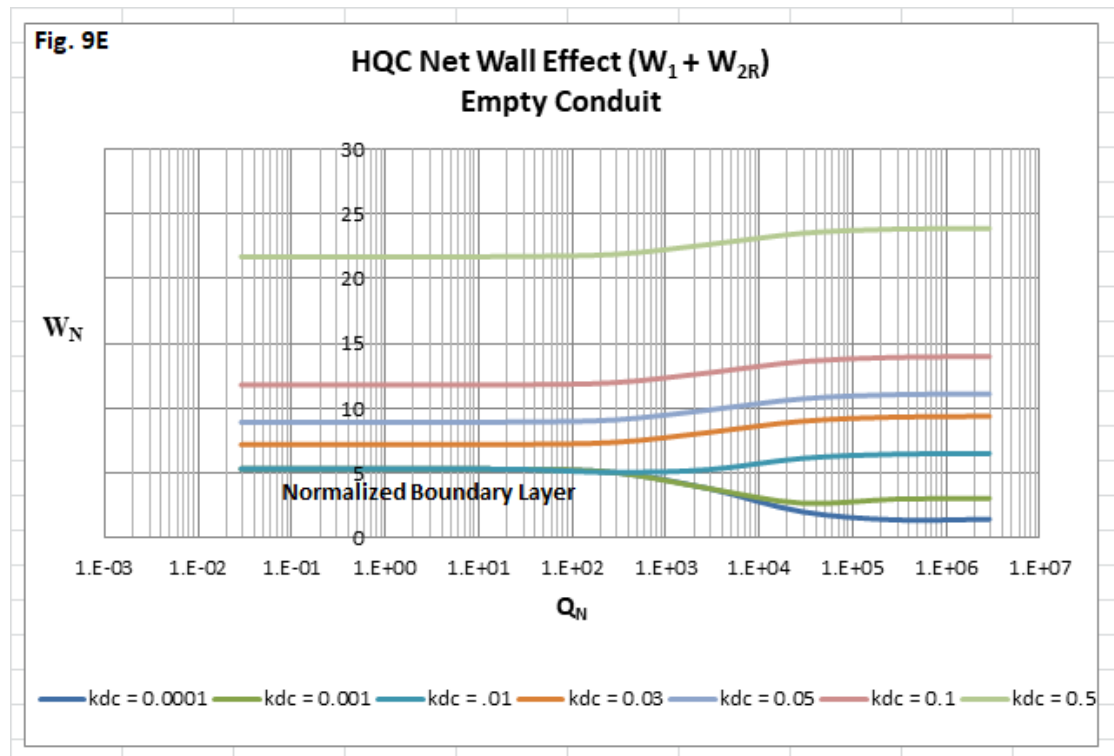

As shown in the plot, the net wall effect, $\mathrm{W}_{\mathrm{N}}$, is the sum of the primary, $\mathrm{W}_{1}$, and residual secondary, $\mathrm{W}_{2 \mathrm{R}}$, wall effects. Note that the normalized boundary layer is apparent in this plot at low values of $\mathrm{Q}_{\mathrm{N}}$.

7.18 Finally, we evaluate the influence of the HQC net wall effect, $\mathrm{W}_{\mathrm{N}}$, on the HQC fluid current. This we accomplish by showing a plot of the HQC fluid current normalization coefficient, $\lambda$, versus the fluid current, $Q_{N}$, in Fig. 9F.

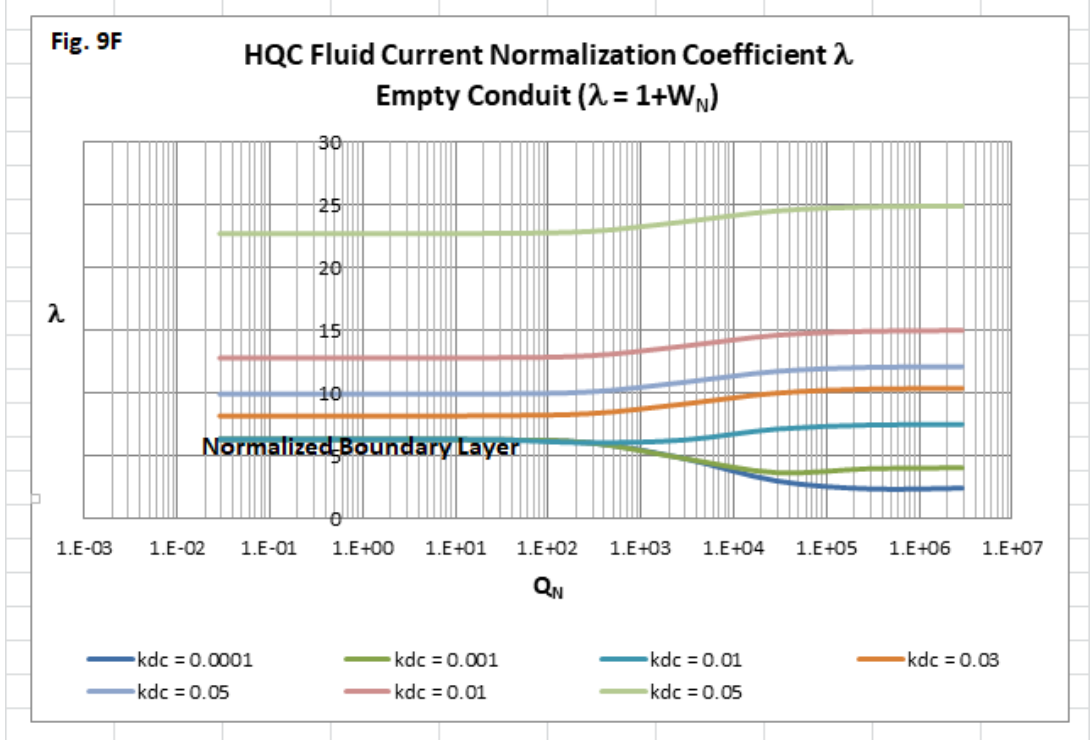


As shown in the plot, the normalized fluid current is relatively constant at low values of $Q_{N}$ and reaches a plateau which may be greater or less than the primary wall effect, commensurate with relative wall roughness.

\section{d. The Fluid Current-Velocity Streamlines}

7.19 When the pressure gradient is unidirectional, as is the case in a walled conduit, the flow only occurs in the direction of the pressure gradient. We can show this by using Math Works ${ }^{\mathrm{TM}}$ in MatLab ${ }^{\mathrm{TM}}$ software to plot the motion of the fluid as shown in Fig. 10A.

Unidirectional Pressure Gradient of Instantaneous Velocity profile in the HQC

Expressed as a function of $\mathrm{t} \boldsymbol{g} \mathrm{x}$

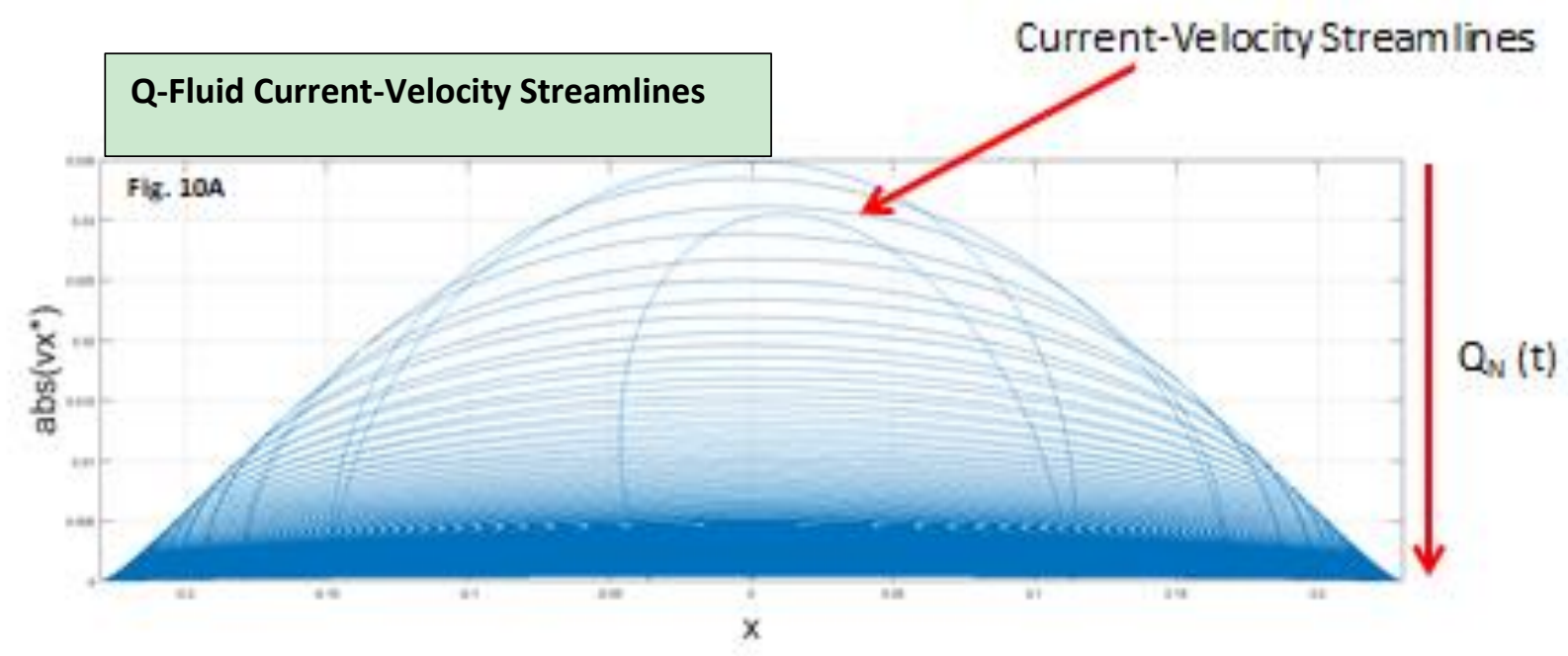

As shown in Fig. 10A, the scale of the motion is defined by the diameter of the HQC, i.e., $d_{c}$. In this example of an empty conduit, the diameter of the HQC is $0.46 \mathrm{~cm}$ and, accordingly, the maximum radius of the fluid flow profile is $y=0.23 \mathrm{~cm}$. Note that the instantaneous speed is zero at both conduit walls $(x=0.23 \mathrm{~cm}$, and $x=-0.23 \mathrm{~cm})$, i.e., the no slip boundary condition and reaches its maximum value in the center of the channel $(\mathrm{x}=0 \mathrm{~cm})$. The flow velocity profile goes from a parabolic type shape at low values of the fluid current $\mathrm{Q}_{\mathrm{N}}(\mathrm{t})$ to almost flat at the highest values of $Q_{N}(t)$. Accordingly, we refer to the velocity lines displayed as "currentvelocity streamlines". 


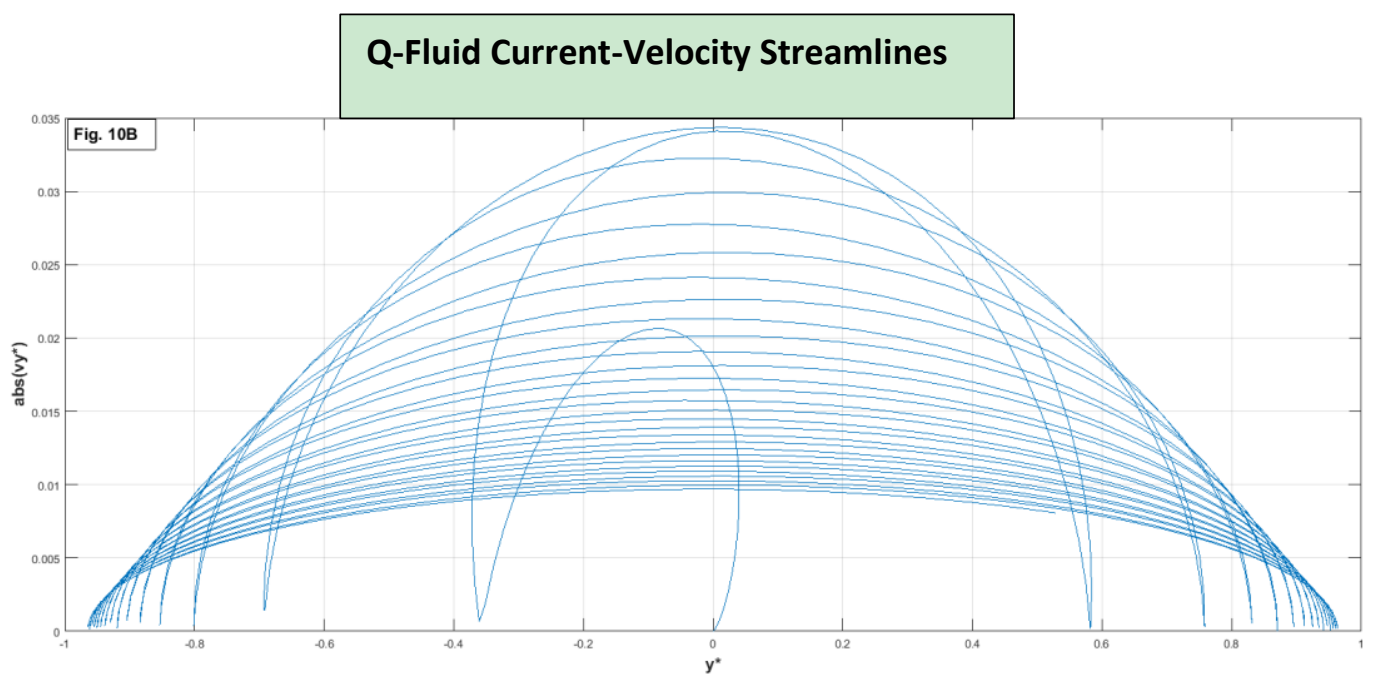

As shown in Fig. 10B, the dimensionless fluid flow profile has the normalized radius of unity as shown in the plot of $\operatorname{abs}\left(\mathrm{v}_{\mathrm{y}}{ }^{*}\right)$ versus $\mathrm{y}^{*}$.

7.20 In the case where the pressure gradient is not uni-directional, we can view the fluid velocity profile in the $\mathrm{x}$ axis plane, as in Fig. 10C.
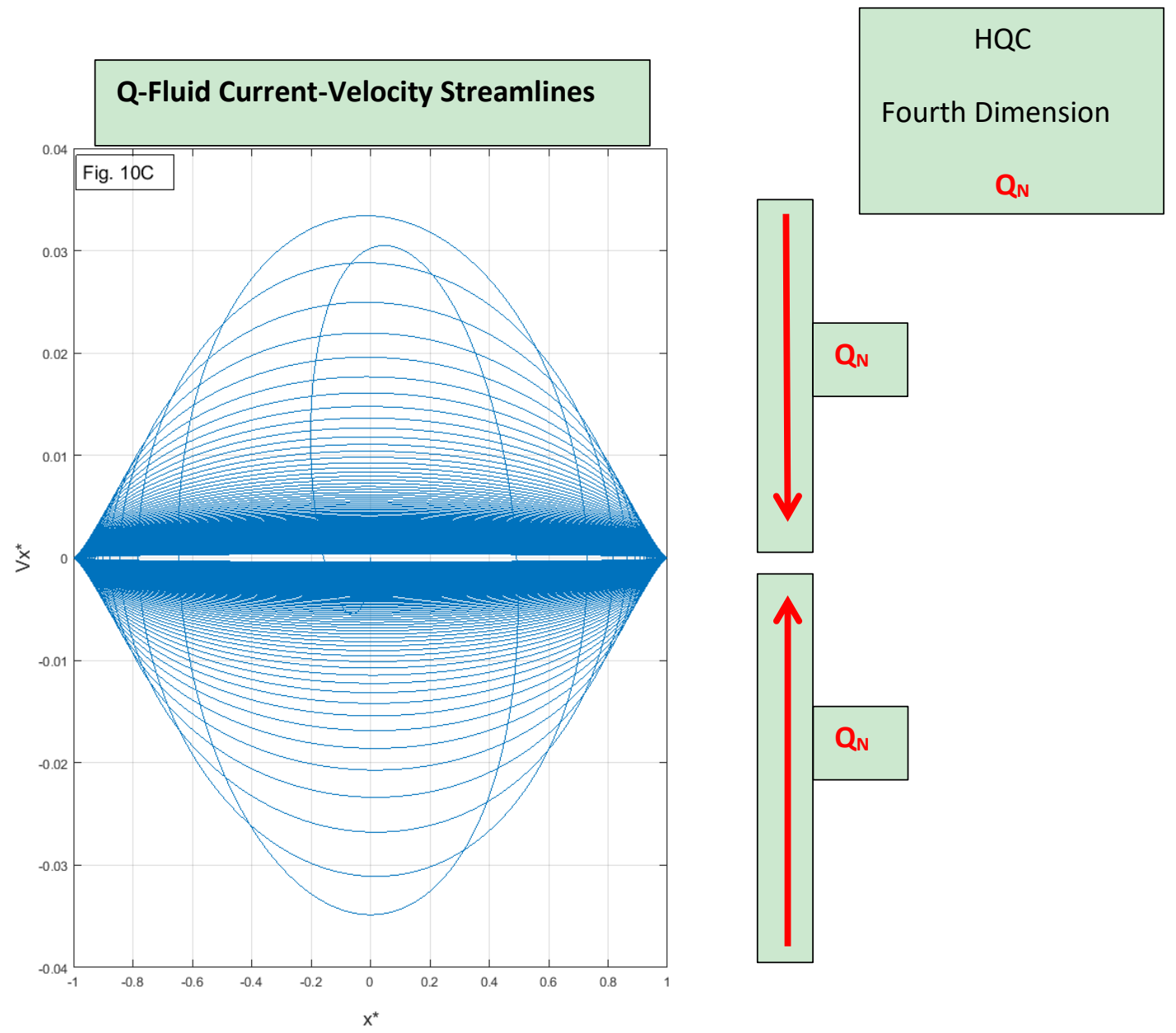
As shown in Fig. 10C, the instantaneous fluid speed is zero at the wall and maximum in the center of the channel. In addition we can see the parabolic motion changing as a function of the value of the $Q_{N}$ number.

7.21 We can also view the velocity profile in three dimensions, as shown in Fig. 10D.

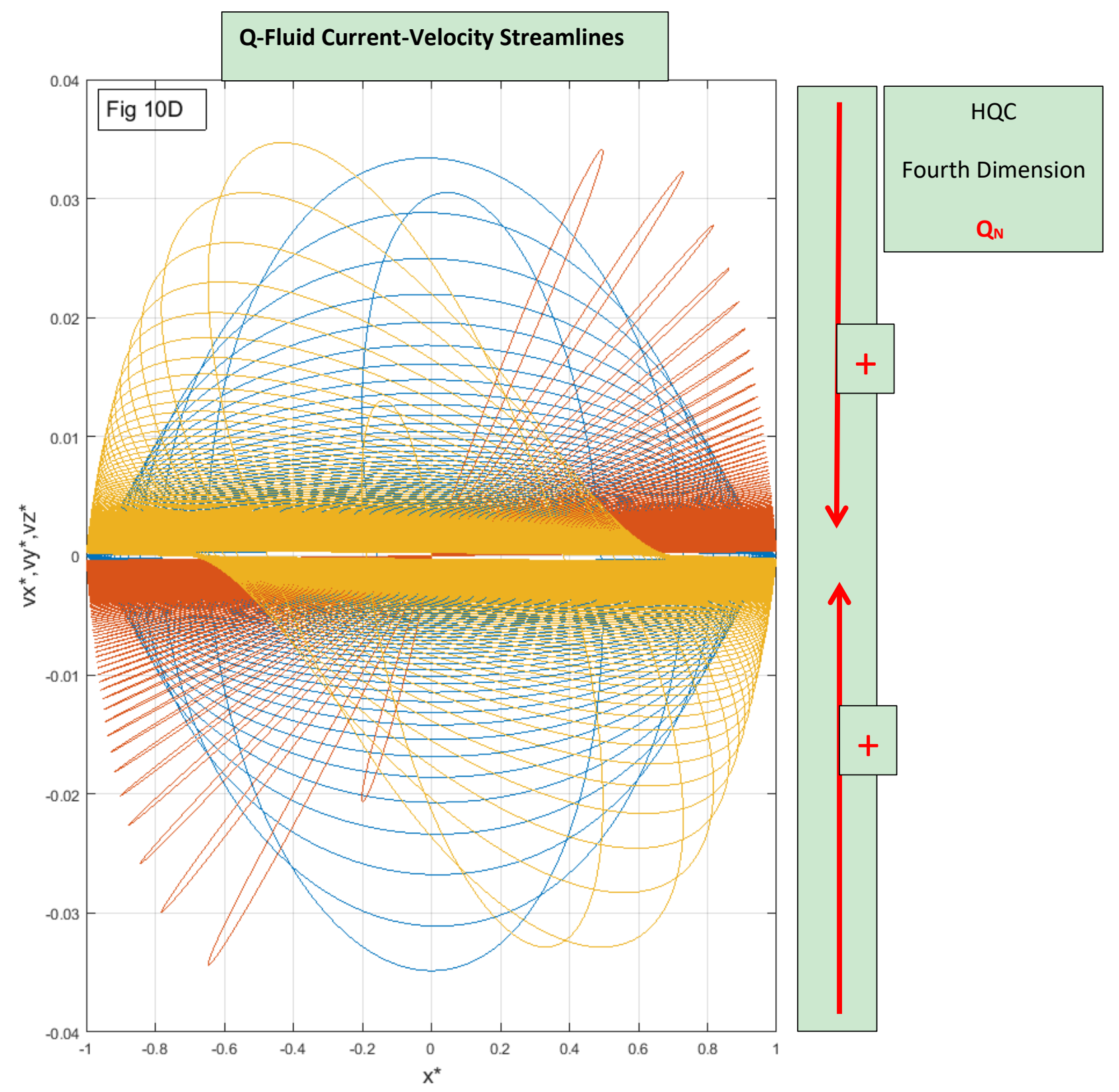

As shown in Fig. 10 D, the flow profile takes on the characteristic shape shown in the plot. 
7.22 Similarly, we can view the fluid velocity profile as a function of fluid current, $Q_{N}$, in the three dimensional plane as shown in Fig. $10 \mathrm{E}$.

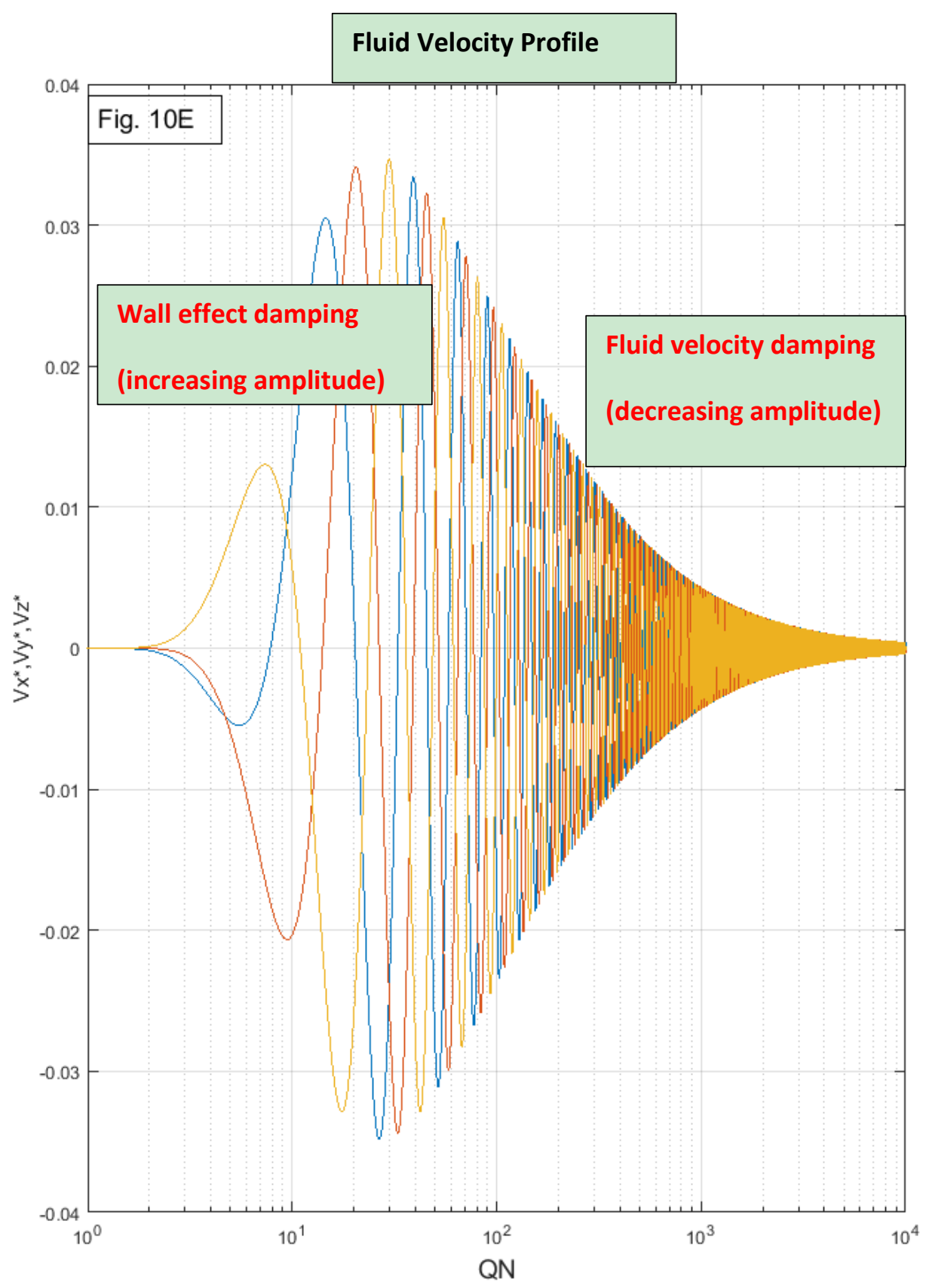

As shown in the plot, the changing fluid flow profile is captured as a function of fluid current using a $\log \mathrm{x}$ axis. The damping effect of wall friction and fluid friction are apparent in this plot. 
7.23 Next we view the instantaneous dimensionless boundary layer as a function of $\mathrm{Q}_{\mathrm{N}}$, as shown in Fig. 10F.

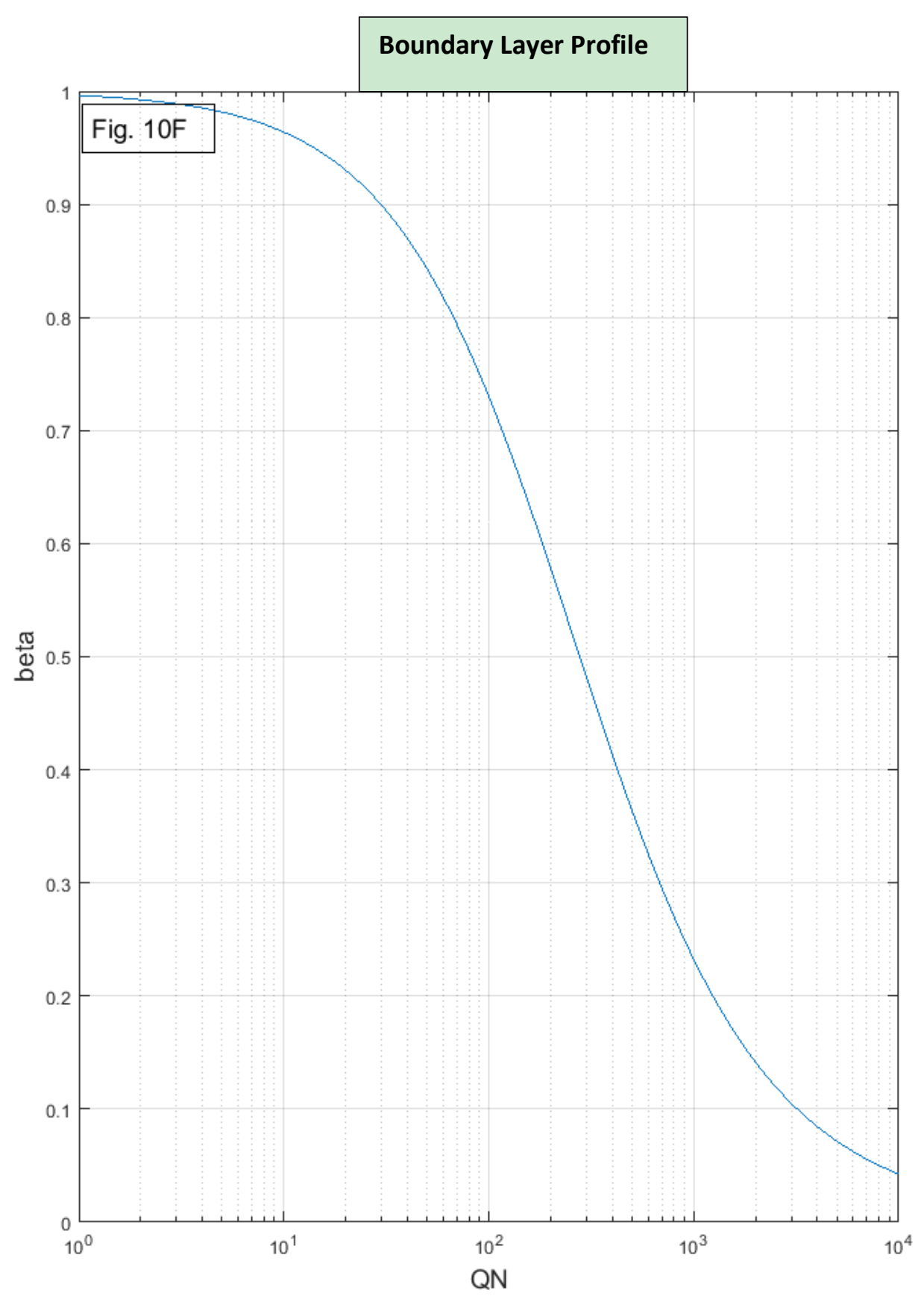

In Fig. 10F we can see the disruption of the fluid wall boundary layer as it goes from unity, at low values of $Q_{N}$, to zero at very high values of $Q_{N}$, on a $\log x$ axis. 
7.24 We can view the important dimensionless parameters of $\beta, \mathrm{P}_{\mathrm{Q}}$ and $\Theta$, as a function of $\mathrm{Q}_{\mathrm{N}}$, in a log-log plot, as shown in Fig. 10G.

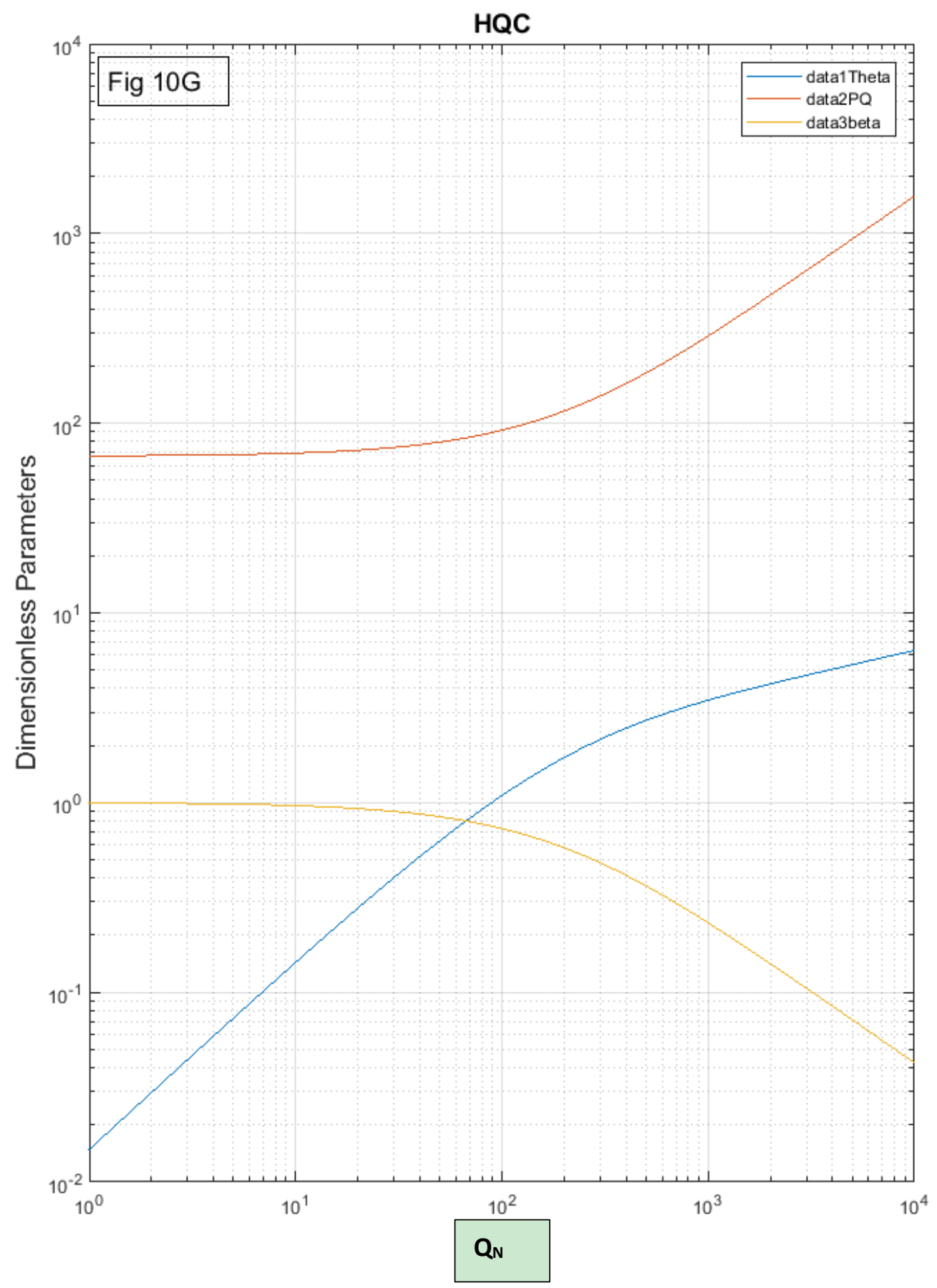

In Fig. 10G we can see how these dimensionless parameters mirror one another, using a log scale on both axes. 
In a dimensional manifestation of scale, we can view the thickness of the boundary layer in Fig. $10 \mathrm{H}$.

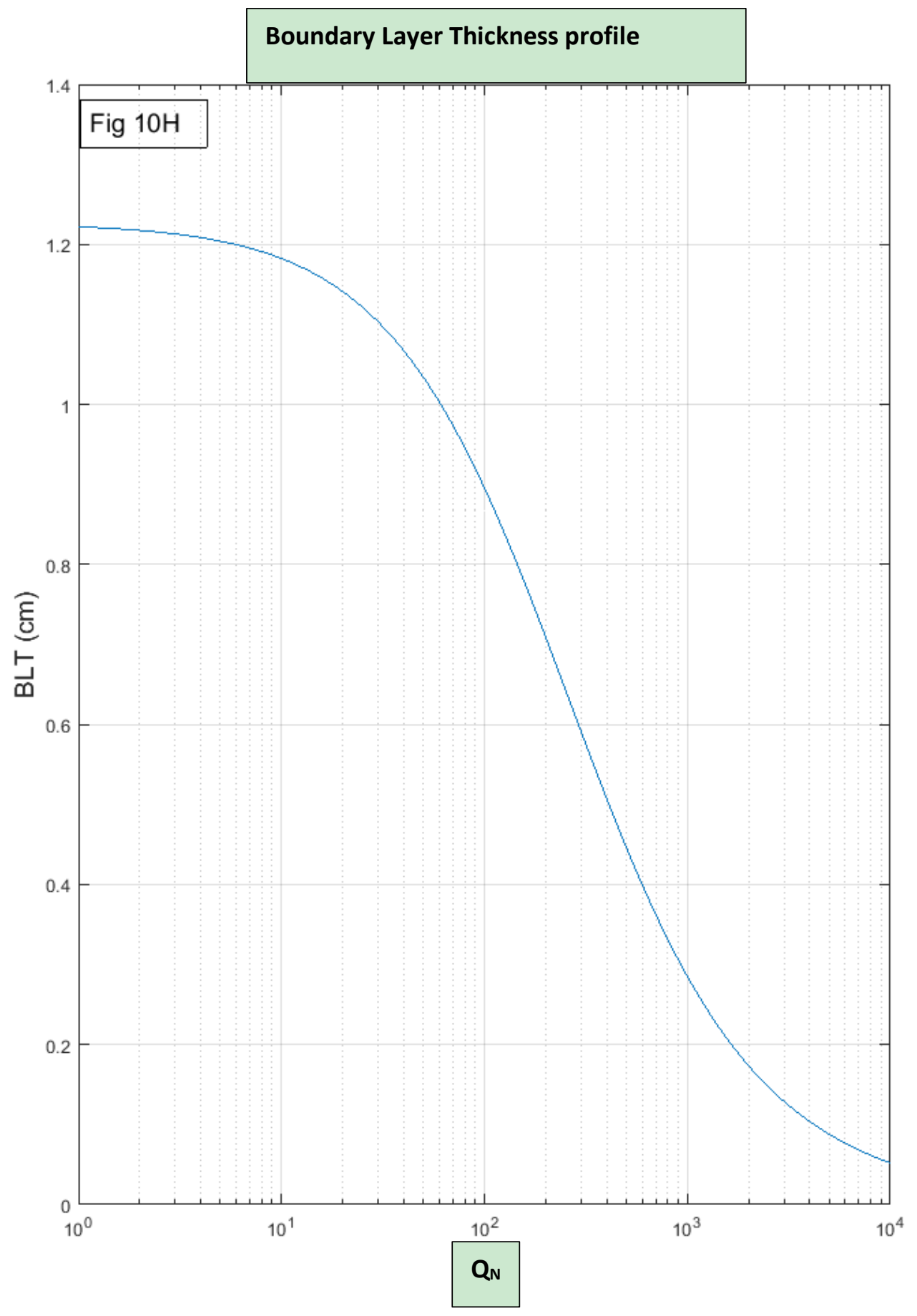

As shown in Fig. 10H, the thickness of the wall boundary layer decreases as a function of $\mathrm{Q}_{\mathrm{N}}$. 
7.25 We can also view the fluid motion in terms of the three parameters of BLT, $\mathrm{x}^{*}$ and $\mathrm{Q}_{\mathrm{N}}$, as shown in Fig. 10J.

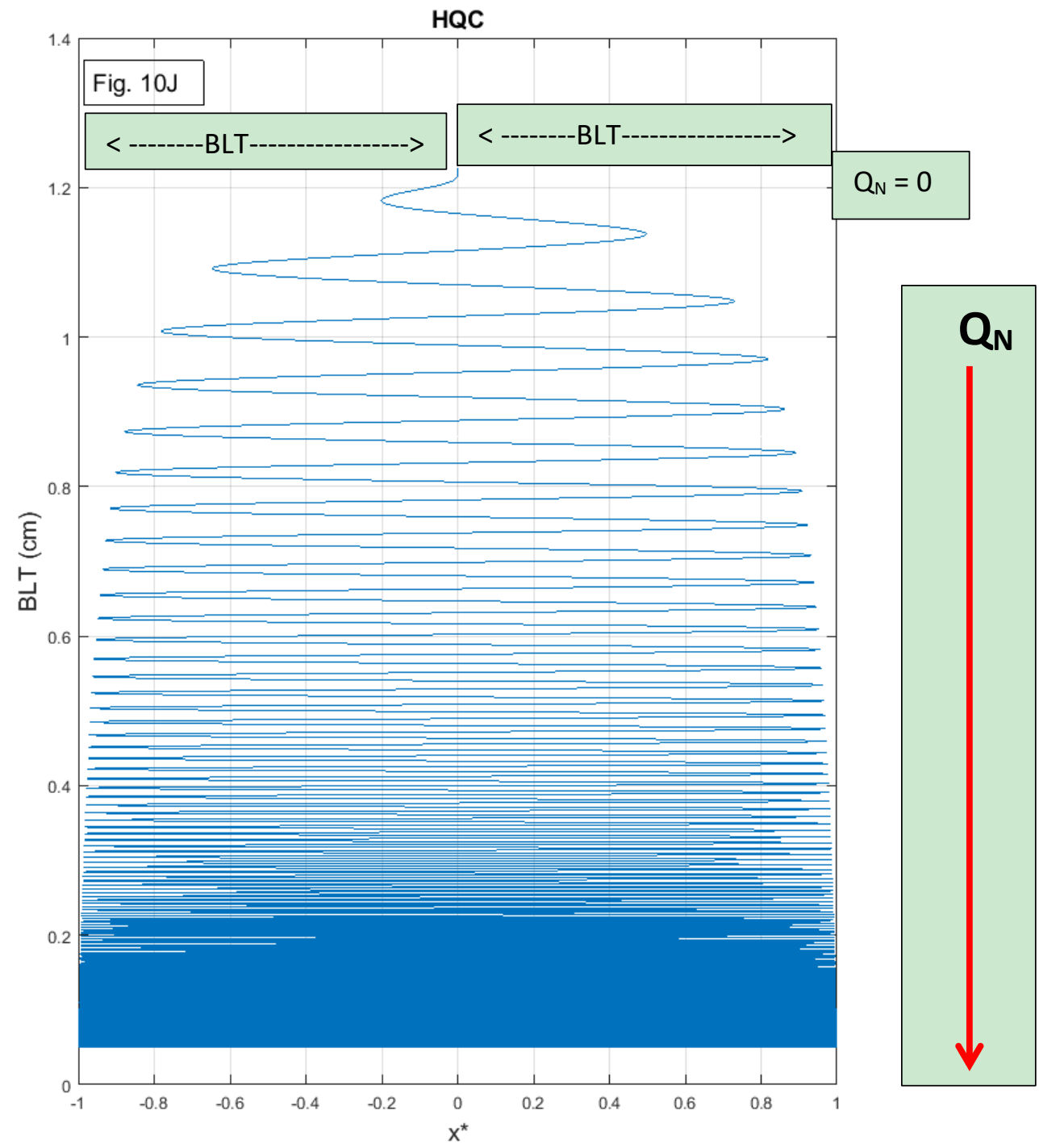

Note that at very low flow rates, the boundary layer consumes almost the entire channel diameter, as shown in Fig. 10J.

The boundary layer thickness is equivalent to the radius of the HQC at time zero, i.e., when the fluid is at rest. Fluid motion begins in the center of the HQC and gradually spirals outward as a function of the fluid current, $Q_{N}$. The boundary later thickness is minimized at the maximum value of $Q_{N}$ reached in any given experiment. Accordingly, one can think of the $Q_{N}$ parameter as a "fourth dimension" when viewing the fluid velocity profile, representing as it does, the movement of elapsed time of the harmonic oscillator. 


\subsection{Laminar", "Transitional", "Turbulent" and "Chaos"}

Finally, the issue of fluid chaos as articulated in many conventional scholarly works demands attention in light of the QFFM [Southerland]. The theory outlined herein contradicts the conventional wisdom that fluid turbulence is merely fluid velocity occurring in a random and unpredictable fashion. On the contrary, the QFFM demonstrates that fluid current is in fact highly structured and totally predictable. We underscore this point in Fig. 10K.

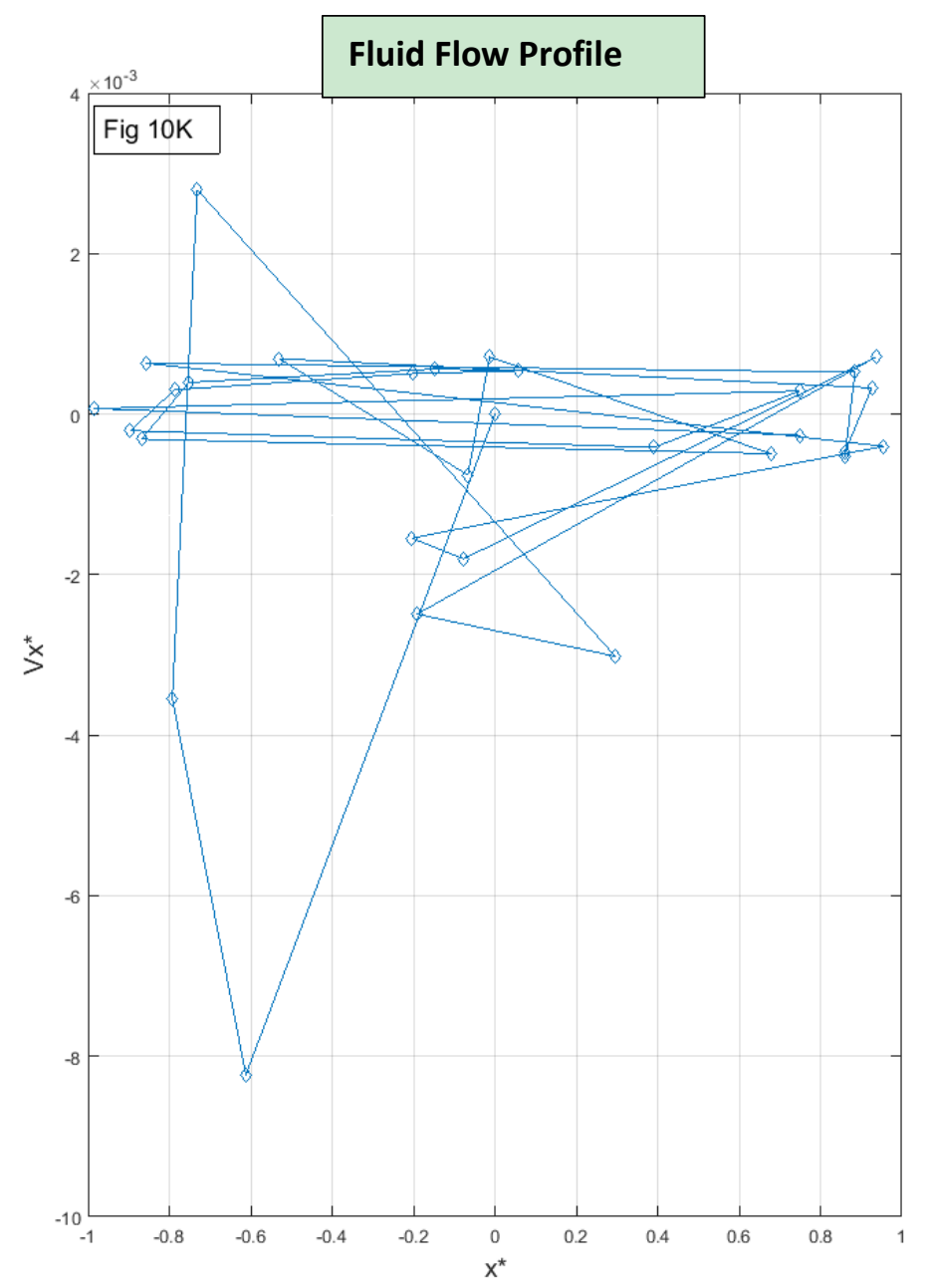

As shown in Fig 10K, we include a plot of the exact same data as that presented in Fig.10C.

The data shown in Fig.10C has a flow rate domain in MatLab ${ }^{\mathrm{TM}}$ of q: $0.01 ; 0.001 ; 300$. In layman's terms, this means that the flow rate varies between a value of $0.01 \mathrm{~mL} / \mathrm{sec}$ and 300 $\mathrm{mL} / \mathrm{sec}$ and that there is a data point recorded every $0.001 \mathrm{~mL} / \mathrm{sec}$, i.e., the measurement interval is $0.001 \mathrm{~mL} / \mathrm{sec}$.

The data shown in Fig. 10K, on the other hand, has a flow rate domain of q: $0.01 ; 10 ; 300$, i.e., the same range of flow rates but a much larger flow rate interval. Actually the flow rate interval is $10 / .001=10,000$, i.e., 10,000 times larger. 
The explanation, therefore, for the "chaotic" view of the fluid instantaneous velocity in Fig. 10K, as opposed to the "highly structured" view of the fluid profile in Fig. 10C, for the exact same data, is due to the fact that there are not enough data points taken in the plot in Fig. 10K to identify the precise motion of the fluid, noted above, the period of the motion in a packed conduit is approximately $158\left(\mathrm{~T}=2 \pi / \omega\right.$, and $\lambda=1 ; \mathrm{T}=16 \pi^{2}=158$ approximately $)$.

In Fig.10C the interval of $0.001 \mathrm{~mL} / \mathrm{sec}$ provides ample data points to clearly outline the motion of the fluid, since this interval represents a "slice" in dimensionless time, $\left(\Delta \mathrm{Q}_{\mathrm{N}}\right)$ much smaller than the period of the motion $\left(\mathrm{Q}_{\mathrm{N}}=158\right)$.

Accordingly, the conventional folklore labels of, "Laminar", "Transitional", "Turbulent” and "Chaotic flow" do not represent scientifically defensible criteria. Rather, they are merely subjective experimental observations which have never been quantified to any scientifically acceptable standard. Among other things, this is why the fluid dynamic landscape is littered with equations and proclamations which purport to set boundaries around each of these ill-defined labels and, consequently and unsurprisingly, none of the proposed boundaries have found universal acceptance. On the other hand, the QFFM replaces all these subjective labels with just one entity "fluid current" and defines it with scientific precision and accuracy. Moreover, it postulates that fluid current is a continuum valid throughout the entire range of flow regimes and, when evaluated with the necessary precision in the fourth dimension, i.e., the interval values of $\Delta \mathrm{Q}_{\mathrm{N}}$, commensurate with the flow embodiment which surrounds it, is highly quantifiable and predictable.

\section{J. Validation of the QFFM and Quinn's Law}

We begin our validation protocol by focusing on the general case of our hypothetical Q channel, the packed conduit. In so doing, we have chosen measured data from 4 third party published studies and 3 from homegrown experiments. The homegrown experiments were necessary to augment the other studies in order to cover the region of higher $Q_{N}$ values, where no third party published studies were available.

\section{a. Packed Conduits}

\subsection{Farkas, et al}

In a 1999 publication by Farkas, Zhong and Guiochon, entitled Validity of Darcy's Law at Low Flow Rates in Liquid Chromatography [29, 30], Farkas and his co-authors report the results of some very exacting measurements which they made to validate Darcy's law. Using a column containing spherical particles packed to a measured external column porosity of 0.399, (Fig. 2 in the paper), the authors measured the column pressure drop and fluid flow rate at flow rates ranging between 0.015 and $0.5 \mathrm{~mL} / \mathrm{min}$ with ethylene glycol as the fluid. They state that the particles in the column are spherical and appear to have gone to extraordinary lengths to obtain an accurate measure of the particle diameter and, of course, as a result of modern techniques of particle size classification, the particle size distribution is narrow. Moreover, they used the technique of inverse size exclusion chromatography to determine the external column porosity which is a very accurate methodology when used in the manner described by these authors for the column under study in this paper. 
The range of modified Reynolds number covered by the measurements in this paper, $5 \times 10^{-5}$ to 5 $\mathrm{x} 10^{-3}$, is so low that we consider it to be not only unique in the literature on bed permeability, but also the most relevant data available pertaining to laminar flow in closed, packed-bed conduits. The authors accomplished this feat by choosing the combination of a high viscosity fluid (ethylene glycol), small spherical particles (10 micron approx.) and low superficial fluid velocity, a consequence of their choice of column diameter in combination with fluid volumetric flow rates.

\subsection{Coulson}

The $\mathrm{PhD}$ thesis of J.M. Coulson, published in 1935 contains 111 pages of single-spaced text and tables, as well as approximately a dozen pages of engineering drawings and hand -plotted graphs [31].

The study involves 10 packed columns of varying particle sizes and porosities. All the particles are perfectly spherical, steel, non-porous ball bearings ranging in diameters from the smallest at 1/16 inch (approx. 1600 micron) to the largest 5/16 inch (approx. 8,000 micron).

The column hardware assembly consisted of a series of 2-inch internal-diameter brass castings, honed to a high finish, and bolted together to form a single column of nine sections. Glass Utube manometers were mounted at the section interfaces to measure pressure drop. The manometers were arranged in a rather complex way using air as a pressurization counterbalance and, depending upon the pressure drop ranges, the U-tubes were filled with either mercury or water. The same column hardware assembly was used for each of the packed columns.

The pressure source was an adjustable weir assembly which moved in a vertical plane thus applying head pressure by virtue of its relative elevation to the column inlet. A circulating pump was used to recycle the effluent back to the weir. The fluid was light oil with a kinematic viscosity of approximately 1 stokes.

Flow rate measurements were taken on a gravimetric basis and the time of displacement was measured by stopwatch. The column length was measured, as was the mass of spheres used for each bed compaction. The mass of each sphere was measured and the volume occupied by the spheres was thus calculated. The column porosity was calculated by subtracting the volume occupied by the spheres from the empty column volume which was calculated from the physical dimensions of the cylinder (column).

The viscosity of the fluid was determined for each measurement by measuring the temperature of the fluid and reading the corresponding viscosity from a temperature/viscosity curve obtained by a Redwood viscometer. Density measurements were taken for each experiment by collecting a sample of the fluid effluent and measuring the density in a hydrometer.

\subsection{Ergun}


Sabri Ergun et al published three seminal papers circa 1950 [32, 33, 34, 35]. In the last of these papers, 1952, Ergun published his now famous Ergun equation which he says was based upon a data base of 640 measurements in packed columns. In all three papers, he included much experimental data, some of which he carried out himself and some of which he took from third party studies. In this paper, we focus on the measurements he made himself for columns which contained both spherical and irregular particles through which he passed nitrogen gas. We include in our selected data base approximately 70 different columns with about 8 individual flow rate measurements for each column. Thus, our data base represents more than 500 individual measurements. All the measurements are in the transitional region of the fluid flow regime, which means that all his pressure drop measurements contained significant contributions from both viscous and kinetic sources.

\subsection{Giddings}

We include as part of our validation the works of J.C Giddings included in his Table 5.3-1 reported on page 209 of his 1965 textbook [36]. This reported data includes permeability measurements of packed chromatographic columns containing both nonporous and porous particles. Importantly, Giddings took advantage of smooth spherical glass bead particles to overcome the difficulty associated with irregular particle shape.

\subsection{HMQ -1}

The column used in this study was a chromatographic column prepared by this author by means of slurry packing under a packing pressure of 5,000 psi approximately, a technique well known in the chromatographic field. The column was made of $316 \mathrm{SS}$ and had the dimensions of $2.1 \mathrm{~mm}$ in diameter and $100 \mathrm{~mm}$ in length. The particles were made of an organic copolymer which was cross-linked to give the particles sufficient rigidity to be run at pressures up to several hundred bars. The particles are known by the commercial name of Oasis and are manufactured by Waters Corporation. The particle size distribution was measured by standard coulter counter analysis and had an average particle size of 30 micron. In addition, the particle size distribution was narrow. External porosity was measured in dichloromethane solvent by inverse size exclusion chromatography and determined to be 0.463 . Pressure drop measurements were taken in the same fluid, dichloromethane, and the flow rate was set using a pre-calibrated HPLC constant flow rate pump with flow rate and pressure drop data recorded in a flow rate range of 1 to 10 $\mathrm{mL} / \mathrm{min}$. This combination of experimental variables provided a range of modified Reynolds numbers of 1 to 8 approximately, which corresponds to the transition region of the flow regime.

\subsection{HMQ -2}

The column used in this study was a chromatographic column, dry packed by this author using the well-known tap and vibrate method of column packing. The column was made of 316 SS and had the dimensions of $1.002 \mathrm{~cm}$ in diameter and $248 \mathrm{~cm}$ in length. The particles were made of an inorganic rigid substrate, non-porous glass beads and were purchased from the Shot glass company. They were spherical in shape as well as smooth and nonporous. The particle size distribution was measured by calibrated calipers in conjunction with microscopy and had an average particle size of 1,000 micron. In addition, the particle size distribution was narrow. The 
physical size of this column was chosen such that it had a large empty column volume (196 mL) which enabled the measurement of external porosity by volumetric means. External porosity was measured by filling the already dry-filled column with water and measuring the volume $(76 \mathrm{~mL})$ with a graduated cylinder. Its external porosity value was thus 0.39 . Pressure drop measurements were taken in water and the flow rate was set using a pre-calibrated HPLC constant flow rate pump with flow rate and pressure drop data recorded in a flow rate range of 4 to $500 \mathrm{~mL} / \mathrm{min}$. This combination of experimental variables provided a range of modified Reynolds numbers of 1 to 175 approximately, which corresponds to the transition region of the flow regime.

\subsection{HMQ-4}

The column used in this study was made of $316 \mathrm{SS}$ and had the dimensions of $1.07 \mathrm{~cm}$ in diameter and $40.6 \mathrm{~cm}$ in length. The particles were made of electro-polished stainless steel ball bearings and were spherical in shape as well as smooth and nonporous. The particle size distribution was measured by calibrated calipers and had an average particle size of $0.952 \mathrm{~cm}$. Because the particles were just slightly smaller than the column diameter, it was easy to count the number of particles required to fill the column. A total of 41 particles filled the column and this resulted in a column external porosity of 0.488 . Pressure drop measurements were taken using air as the fluid medium and a very sensitive calibrated pressure transducer. The flow rate was provided by a calibrated constant volume gas syringe pump used for experiments related to human lung performance. This combination of experimental variables provides a range of $\mathrm{Q}_{\mathrm{N}}$ values of $1 \times 10^{-4}$ to $1 \times 10^{5}$, a total range of 9 orders of magnitude, which range represents sufficient values to establish the overall shape of the plotted curve of $\Theta \vee Q_{N}$.

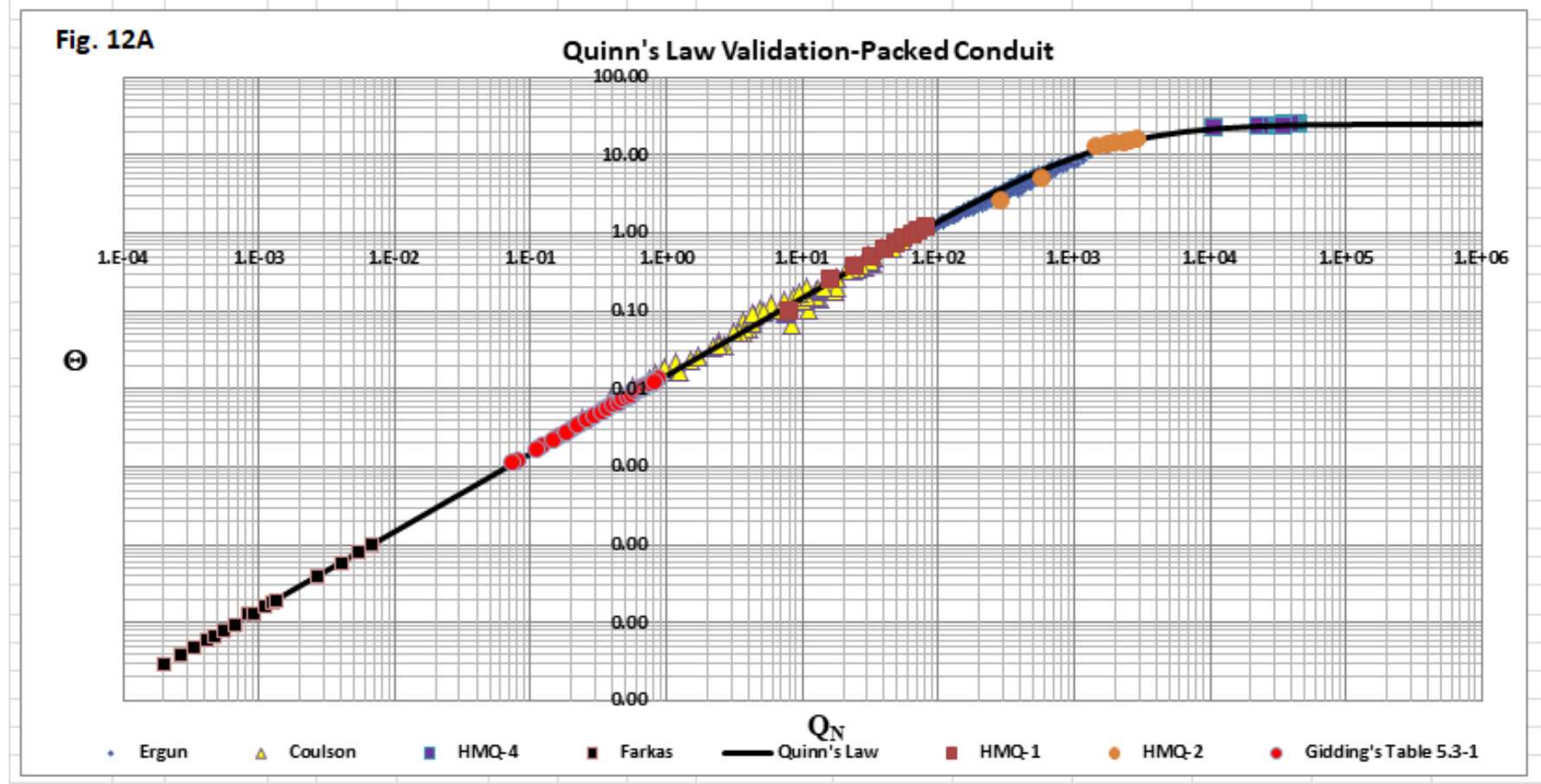




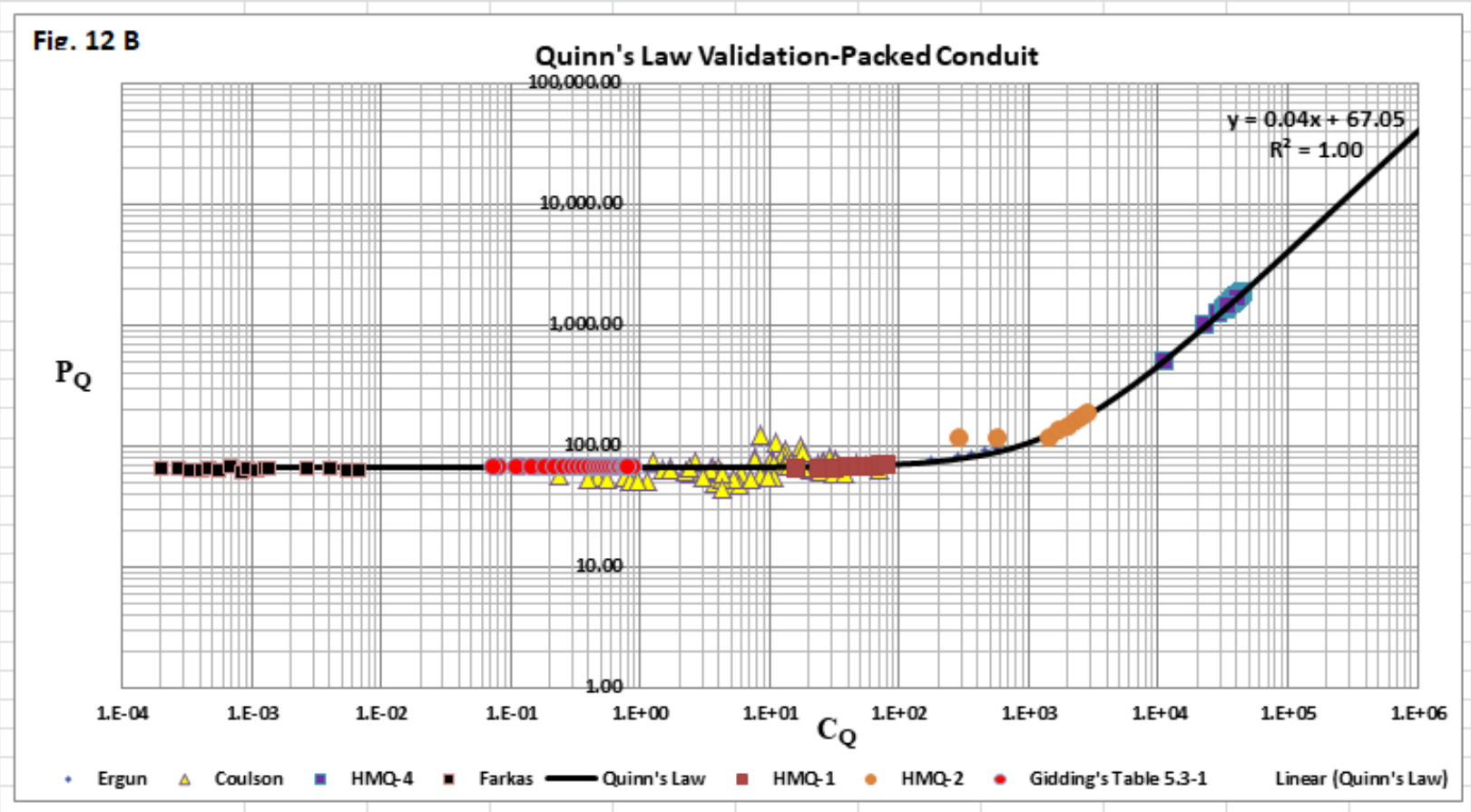

As shown in Fig.12A and B, the empirical data for this data set of packed conduits fits extremely well to the theoretical curve for the QFFM as well as for Quinn' Law, for all measured data.

\section{b. Empty Conduit-Smooth Walls.}

\subsection{The Princeton Study}

What we call "The Princeton Study" herein is a compilation of two papers published between 2004 and 2005 [37, 38]. The 2005 paper contains a brief abstract which we repeat herein; "The friction factor relationship for high-Reynolds-number fully developed turbulent pipe flow is investigated using two sets of data from the Princeton Super pipe in the range $31 \times 10^{3} \leq \mathrm{R}_{\mathrm{ep}} \leq$ $35 \times 10^{6}$. The constants of Brandt's 'universal' friction factor relationship are shown to be accurate over only a limited Reynolds-number range and unsuitable for extrapolation to high Reynolds numbers. New constants, based on a logarithmic overlap in the mean velocity, are found to represent the high-Reynolds-number data to within $0.5 \%$, and yield a value for the von Karman constant that is consistent with the mean velocity profiles themselves. The use of a generalized logarithmic law in the mean velocity is also examined. A general friction factor relationship is proposed that predicts all the data to within $1.4 \%$ and agrees with the Blasius relationship for low Reynolds numbers to within $2 \%$ ".

\subsection{The Oregon Study}

The Oregon study can be found in a 2002 publication by Oregon University scientists [39]. The paper contains a brief abstract which we repeat herein; "We demonstrate that an unusually small pipe flow apparatus using both liquid helium and room temperature gases can span an enormous range of Reynolds numbers. This paper describes the construction and operation of the apparatus in some detail. A wide range of Reynolds numbers is an advantage in any experiment seeking to 
establish scaling laws. This experiment also adds to evidence already in hand that the normal phase of liquid helium is a Navies-Stokes fluid. Finally, we explore recent questions concerning the influence of molecular motions on the transition to turbulence (Muriel 1998) and are unable to observe any influence".

\subsection{The Nikuradze Smooth Wall Data}

The data in this study is taken from a circa 1930 paper by J. Nikuradse [40]. The study involved the flow of turbulent water through "smooth" test pipe sections of drawn brass construction and unspecified inner wall finish. The range of Reynolds number studied reached as high as one million approximately. Pipe diameters ranging from 1 to $10 \mathrm{~cm}$ were studied. The data lies in the region of fully developed turbulent flow for the most part.

\section{c. Homegrown Experiments in Empty Conduits-Smooth Walls}

8.20 In order to supplement our validation using third party published data with our own homegrown experiments, we carried out a series of measurements in empty capillaries [41]. In every experiment, we measured the temperature, flow rate and pressure drop at as many flow rates as was reasonably possible given the constraints of the pump, i.e. maximum pressure, minimum flow rate and pump power. The pressure drop was recorded by means of a calibrated pressure transducer purchased from Omega, Model \# PX409-250DWU5V. It had a pressure range of 0-250 psi and ran under a 24V DC power supply. The flow rate was measured for each recorded pressure drop by means of a stop watch and graduated cylinder. The time interval over which the measurement was taken varied with the flow rate-larger for low flow rates and smaller for high flow rates. The temperature of the fluid was recorded by means of a thermocouple purchased from Omega, Model \# TCK-NPT-72.

The liquid pump was manufactured by Fluid-o-Tech (Italy), Model \# FG204XDO(P.T)T1000. It is an external gear pump, 0-5V, 300-5,000 rpm delivering pulseless flow rate under a constant pressure. The flow rate of the pump was controlled by means of a laptop computer running under a software control package manufactured by National Instruments. The pump had a flow rate range of 100-1600 $\mathrm{mL} / \mathrm{min}$ and a pressure maximum rating of circa 200 psi. This range of flow rates was further enhanced at lower flow rate values by the use of our recycle valve, which was used to shunt the flow between the device under study and the recycle line.

\subsection{HMQ-10}

In this experiment, we chose to evaluate the permeability of a commercially available empty capillary made of Peek plastic, an article of commerce in the HPLC industry, which had a nominal diameter of 0.02 inches. The fluid used was water and we chose to evaluate two different lengths, $100 \mathrm{~cm}$ and $726 \mathrm{~cm}$, in order to be able to exploit different modified Reynolds number ranges of values.

\subsection{HMQ-11}


In this experiment, we chose a Peek capillary of nominal diameter 0.03 inches and lengths of 100 and $700 \mathrm{~cm}$. In this experiment we also included in our measurements two different fluids, water and Glycol. The viscosity of the water was 0.01 poise and the density was $1.0 \mathrm{~g} / \mathrm{mL}$. The viscosity for the Glycol solution was 0.38 poise and the density was $1.14 \mathrm{~g} / \mathrm{mL}$.

\subsection{HMQ-12}

In this experiment, we chose a stainless steel capillary of nominal diameter 0.07 inches x 66.5 $\mathrm{cm}$ in length. We used both water and glycol as the test fluids.

\subsection{HMQ-13}

In this experiment, we chose a stainless steel capillary of nominal diameter 0.08 inches x 31.75 $\mathrm{cm}$ in length using, again, both water and glycol as the test fluids.

This compilation of measured data provides a range of $Q_{N}$ values of $1 \times 10^{-1}$ to $4 \times 10^{6}$, a total range of 7 orders of magnitude, which range represents sufficient values to establish the overall shape of the plotted curve of $\Theta v Q_{N}$.

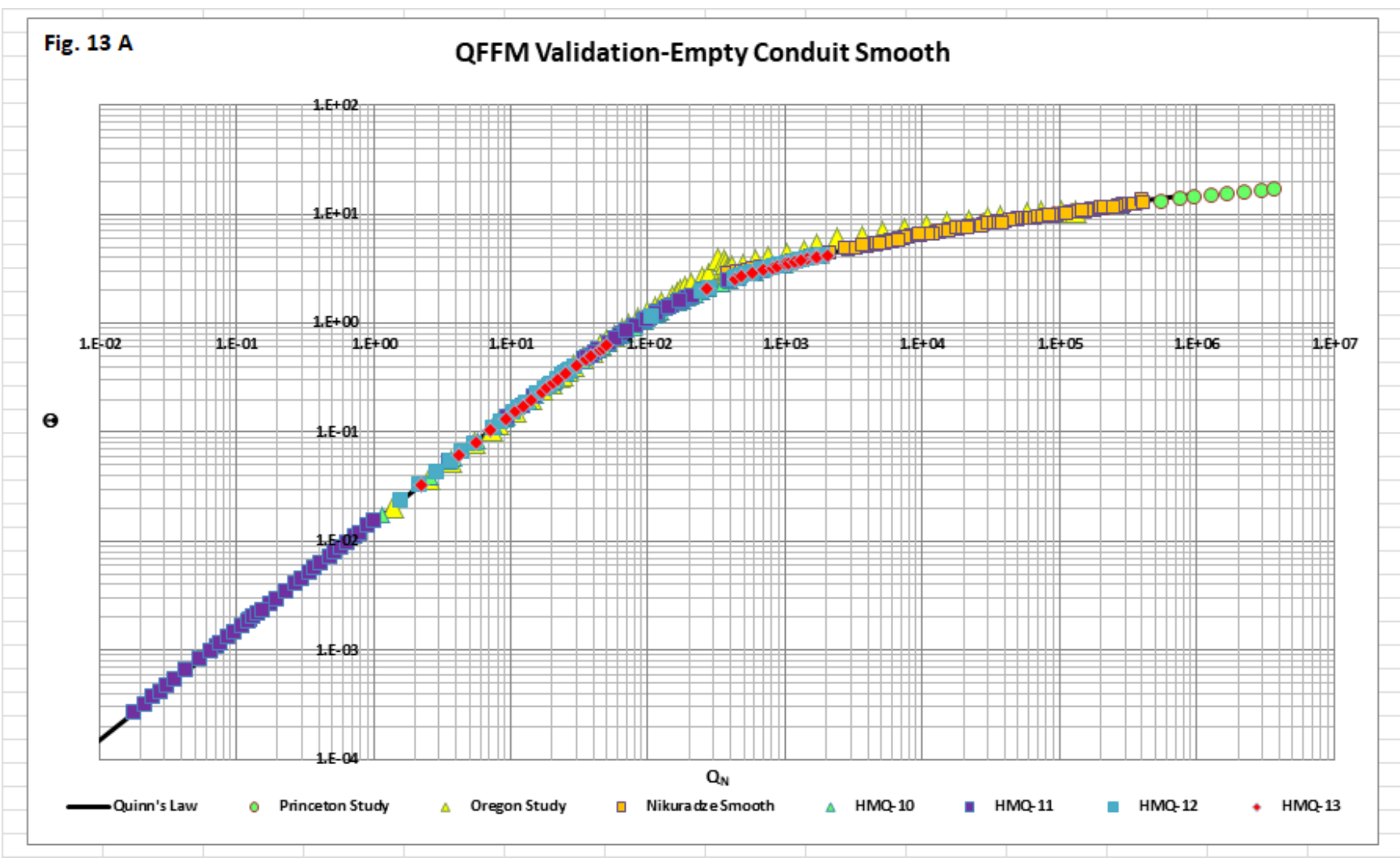




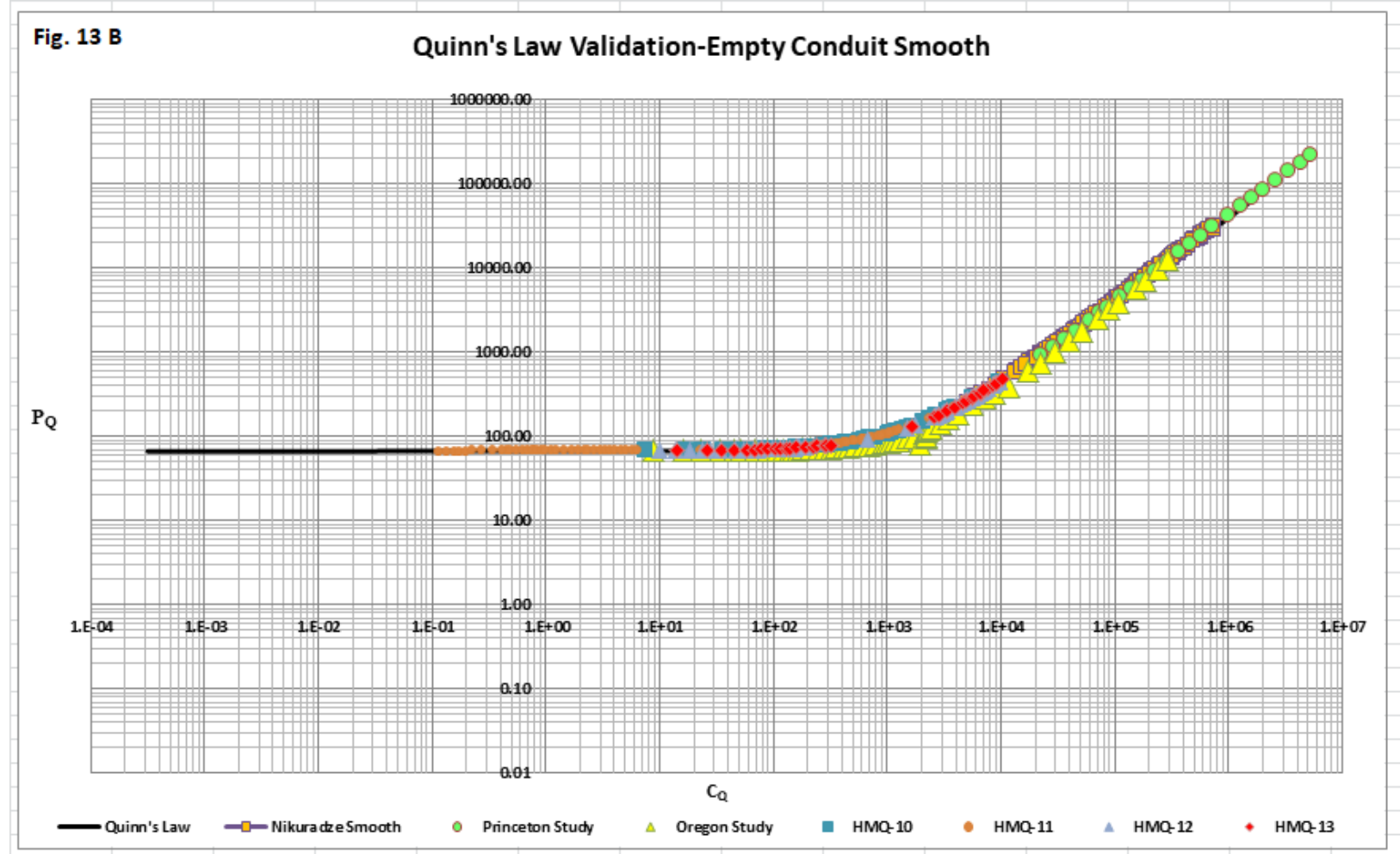

As shown in Fig.13A and B, the empirical data for the smooth empty conduits evaluated, fits extremely well to the theoretical curve for the QFFM as well as for Quinn' Law, for all measured data.

\section{d. Empty Conduits-Roughened Walls}

\subsection{Nikuradze Data}

The data in this study is taken from a circa 1933 paper by J. Nikuradze [42]. The study involved the flow of turbulent water through pipes consisting of drawn brass tubes the inner walls of which were roughened by the deposition of particles of sand. The range of Reynolds numbers studied reached as high as one million approximately. Pipe diameters ranging from 2 to $10 \mathrm{~cm}$ were studied using varying degrees of inner wall roughness. This study is considered the gold standard against which all roughened pipe data is compared.

The combination of experimental variables involved in this study provides a range of $Q_{N}$ values of $5 \times 10^{1}$ to $2 \times 10^{5}$, a total range of 6 orders of magnitude, which range represents sufficient values to establish the overall shape of the plotted curve of $\Theta$ versus $Q_{N}$ [See Fig. 14]. 


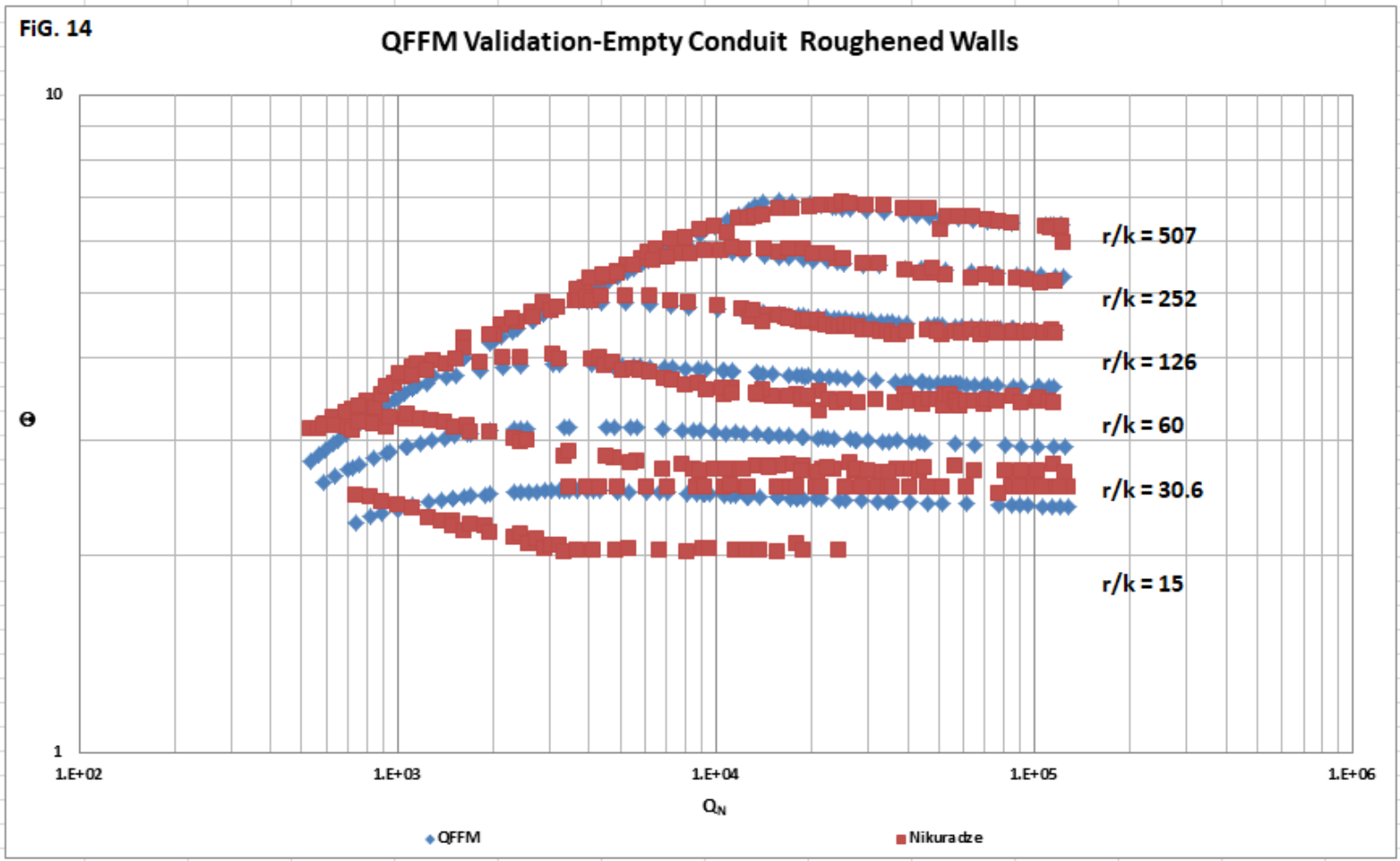

As shown in Fig.14, the empirical data fits extremely well to the theoretical curve for the QFFM for most of the measured data (Nikuradze's r/k values shown). The fit is not so good at the higher relative roughness values due to deficiencies in the experimental procedure as acknowledged in the original publication by Nikuradze himself.

Note that in our Fig.14 herein, we show no data extending into the laminar flow region at low values of $Q_{N}$. This is because we cannot find within the four corners of Nikuradze's publication any measurements taken at low Reynolds numbers. In Fig.9 of the original paper on page 6, written in German, Nikuradze's plot shows a straight line through the laminar region connected to the turbulent region by the now famous "dip/rise" in the line, the so-called "Nikuradze inflection profile". We believe that these plotted points on the graph were added by Nikuradze to make it appear as though his roughened data was a continuation of the conventional wisdom for laminar flow, but that it did not represent any of the experimental measurements reported in his paper.

Conventional folklore concerning the so-called "Nikuradze inflection profile" is awash with hysteria and, the scientific literature is inundated with replicas of Nikuradze's Fig. 9 plot [43, 44], as well as fake replicas of the plot, mostly by mathematicians, who have used advanced mathematical algorithms in misguided attempts to rationalize Nikuradze's supposed measurements connecting the laminar and fully turbulent regions of the flow regime [45]. The reality is that Nikuradze never measured any such inflection point. He is guilty of supplementing his measured data using roughened pipes in the fully turbulent regime with theoretically projected dogma relating to the laminar flow regime, on the same plot, without ever articulating the different origins of the plotted data. 
We also found a discrepancy of a factor of 1,000 in the units of pressure drop data reported in the tables of data in the original paper. We cannot be sure of where this error came from, but it is conceivable that it was simply a unit conversion error made by Nikuradze in preparing the manuscript for publication.

We digress here to explain the true nature of the transition from the so-called laminar to the fully turbulent flow regime using the QFFM. As shown in Fig. 14A, we show that in our worked example for a packed conduit, there is a well-defined profile, free of any inflection or other disturbance, for relative roughness coefficients in the range of $0 \leq \mathrm{k}_{\mathrm{dc}} \leq 0.5$, which corresponds to values of $1 \leq \lambda \leq 25$. This range encompasses all of Nikuradze's roughened data.

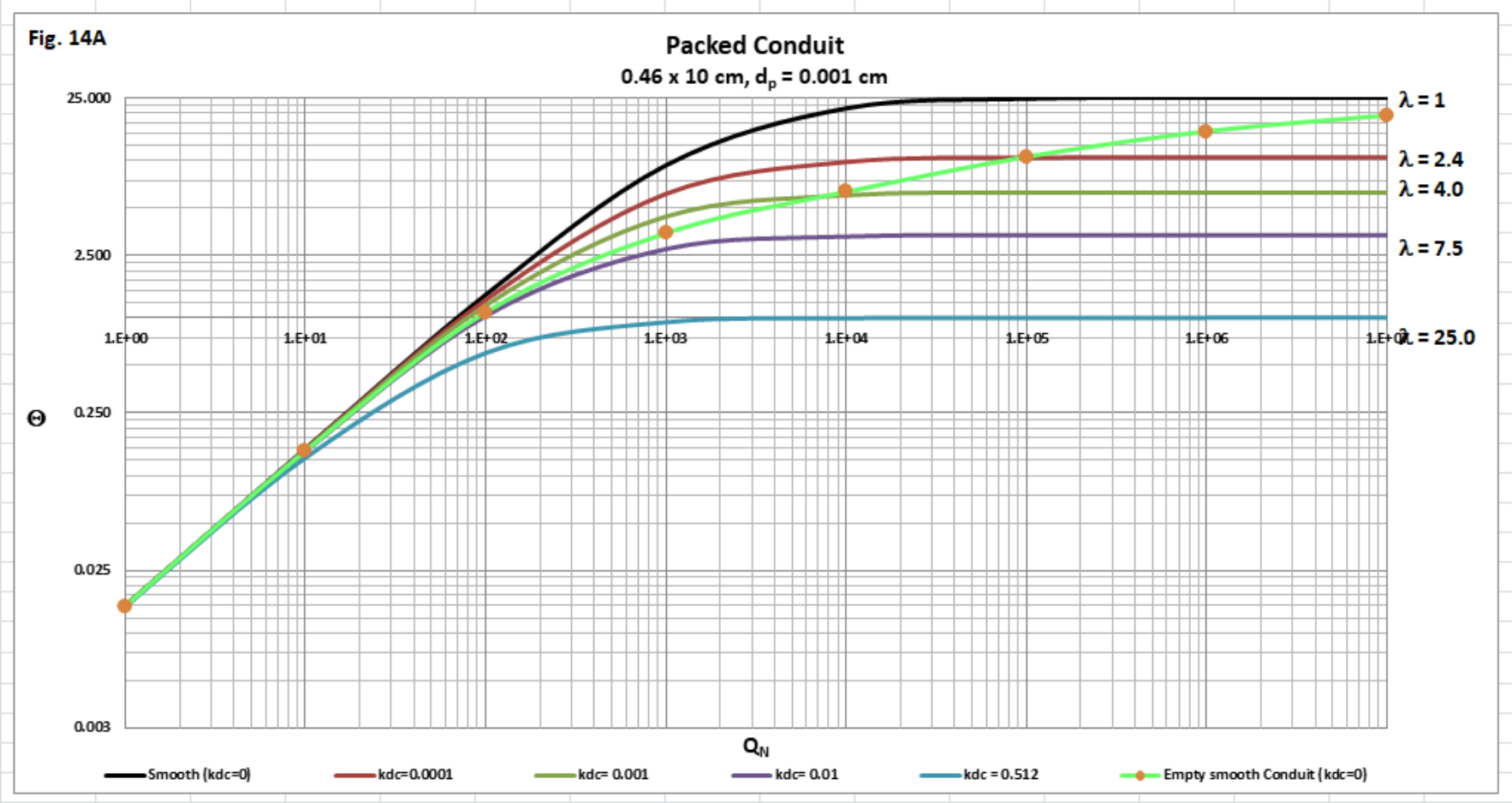

Because the primary wall effect is negligible in the packed column of our worked example, the boundary layer is infinitesimally thin, even at low values of $Q_{N}$, which establishes the baseline for the entire fluid flow regime ( $\omega_{0}=1 / 8 \pi$, the base frequency of the harmonic oscillator). By comparison, as shown also in Fig. 14A, the overlay of our worked example for the corresponding smooth-walled empty conduit, $\mathrm{k}_{\mathrm{dc}}=0$, we can see that even though there is a significant primary wall effect, the boundary layer, which is initially very significant, gradually dissipates at higher values of $\mathrm{Q}_{\mathrm{N}}$, but does so in a very controlled manner, also without any inflection or other disturbances in the profile.

Additionally, as shown in Fig. 14B for the corresponding empty conduit, we can see that, in contrast to the packed column, even though the boundary layer delays the impact of the wall roughness as a function of $Q_{N}$, it does so in a very gradual well-defined manner without any inflection or other points of discontinuity. 


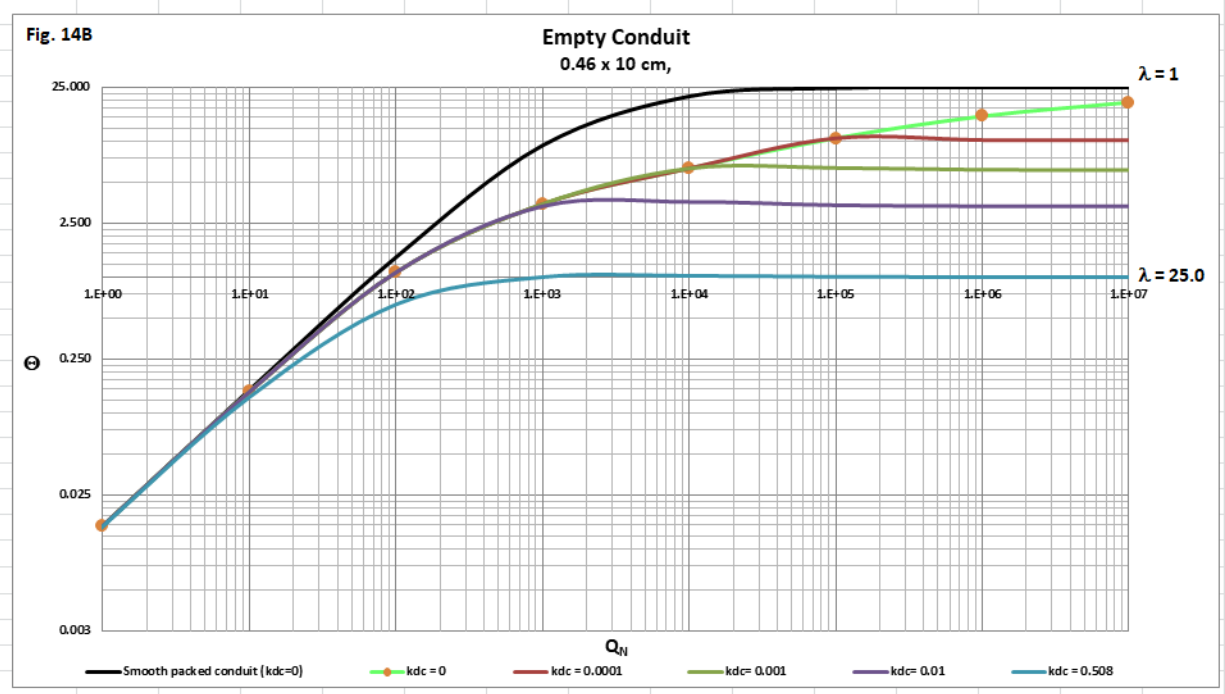

Importantly, as shown in Fig. 14C, when we overlay both portions of Nikuradze's measurements, i.e. the smooth and roughened pipe data, with our worked example for an empty conduit, we can see the orderly progression from the so-called laminar regime, where smooth and roughened pipes are indistinguishable due to the impact of the boundary layer, to the so-called fully turbulent regime, where roughened pipe data deviates from smooth walled data according to the value of $\lambda$, which is due to the overall net wall effect. The line for $\lambda=1$ is shown in the plot as a reference marker.

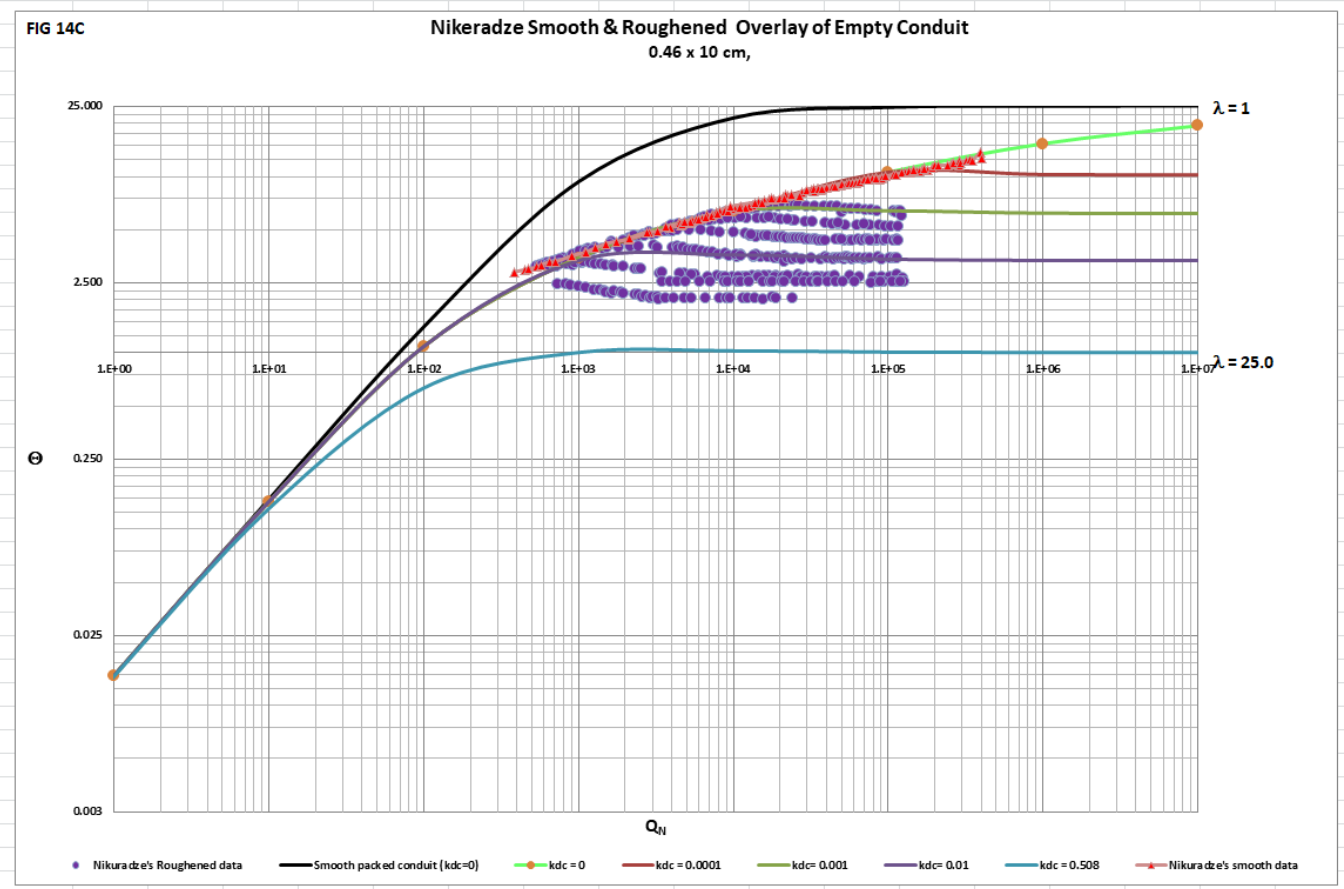

Accordingly, in direct contrast to the "Nikuradze inflection profile" enthusiasts, we dismiss in its entirety any notion that there is a "discontinuity" in the fluid current as the fluid flow rate accelerates from rest to exceedingly large values. 


\section{All Conduits in the same frame of reference}

8.26 Finally, a major benefit of the QFFM is that we can view all the experimental data used in our validation protocol for both packed and empty conduits in the same frame of reference.

Firstly we view the entire validation data as a plot of $\Theta$ versus $Q_{N}$, in Fig. 15A.

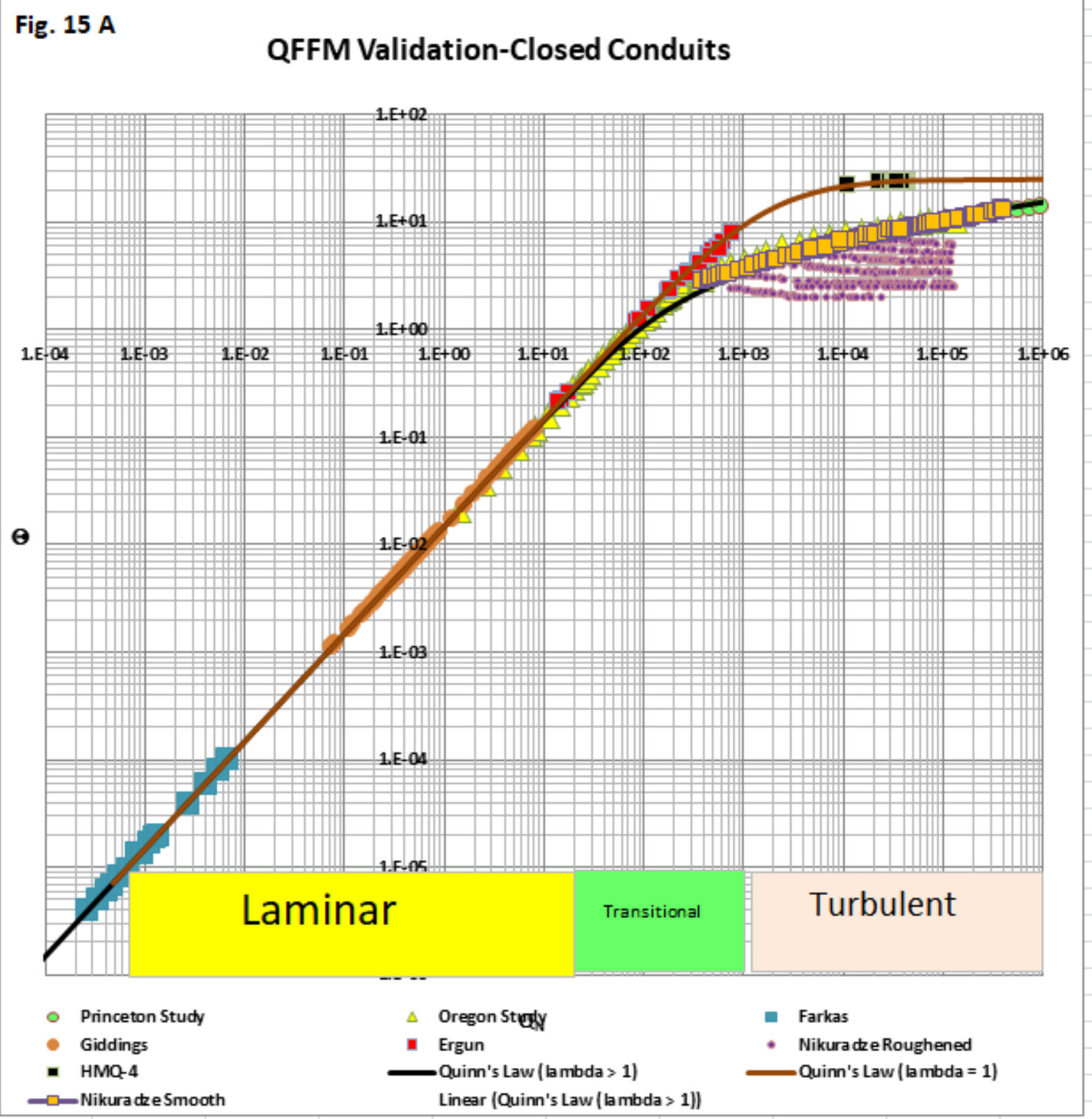

As shown in Fig. 15A, this frame of reference differentiates between packed and empty conduits, on the one hand, and between smooth and roughened walls, on the other. 
8.27 Alternatively, we can view the entire validation data in the linear format of Quinn's Law, as a plot of $\mathrm{P}_{\mathrm{Q}}$ versus $\mathrm{C}_{\mathrm{Q}}$, as shown in Fig. 15B.

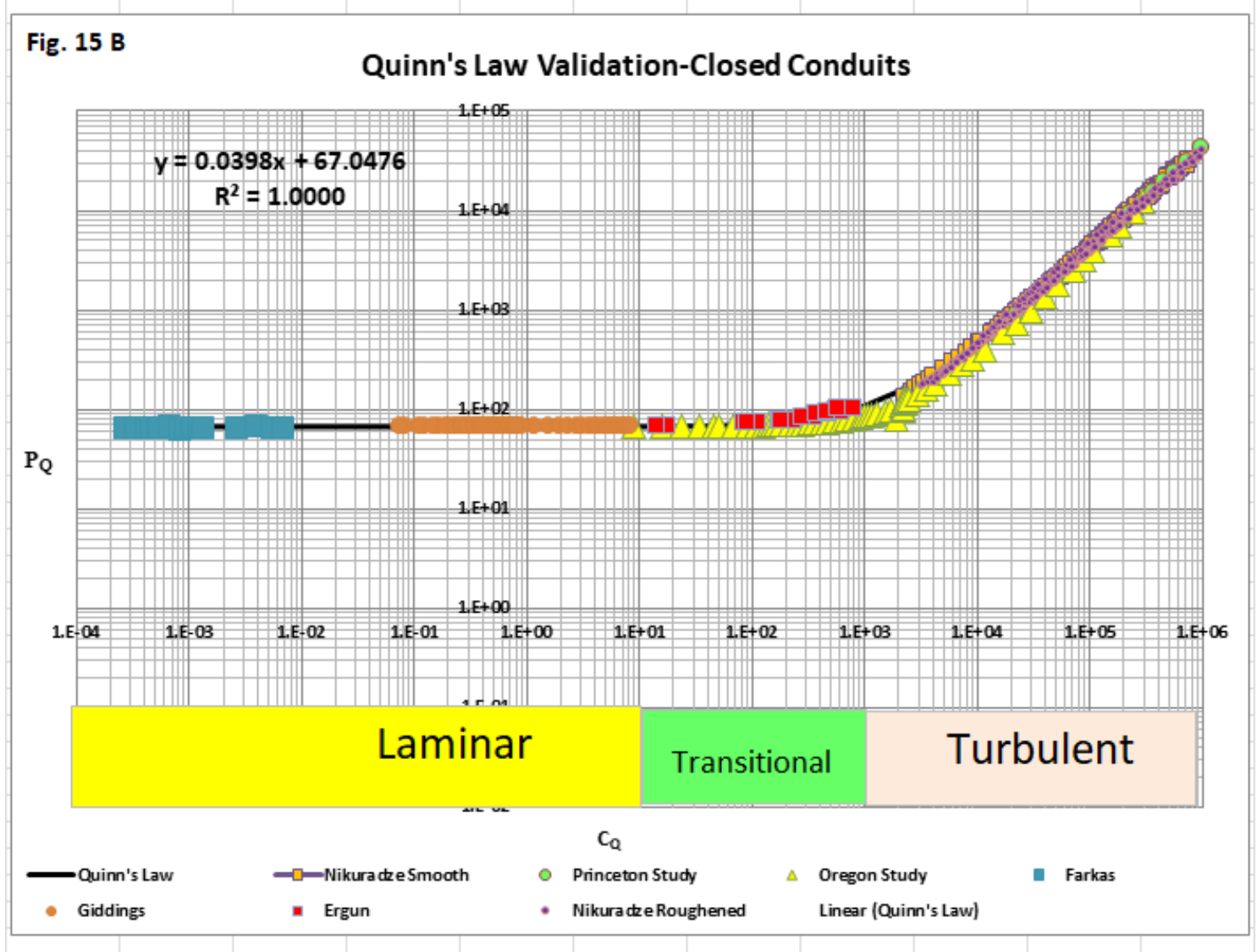

As shown in Figs. 15A \&B, the regions of the subjective fluid flow regime, i.e. laminar, transitional and turbulent, are clearly marked on the $\mathrm{x}$ axis. We do not suggest that the ranges of values of $Q_{N}$ are accurate for each of these designated regimes of flow, since we contend that these labels are of "qualitative" value at best. The plot is $\log$-log to facilitate the data points for both packed and empty conduits over an extremely wide range of modified Reynolds numbers, and all of which fall on a straight line of slope $1 /(8 \pi)$ and intercept $64 \pi / 3$.

The QFFM, therefore, teaches that every experiment carried out in a closed conduit, whether packed with solid particles or devoid of solid particles (a capillary or empty conduit), must fall on the straight line shown in Fig. 15B. Thus, this teaching removes all ambiguity in the pressure/fluid flow relationship in closed conduits and, in addition, defines precisely the role of each flow embodiment parameter as well as that of the flowing fluid. The only source of error remaining is related to the accuracy of a particular measurement technique, i.e., where exactly on the line a particular experimental result should fall.

Accordingly, for the first time in the history of fluid dynamic experiments, the only arguments, which are valid pertaining to fluid flow in closed conduits, are those which are related to the precise value of the particular measured parameters, not the fundamentals underlying the relationship between the driving force $(\Delta \mathrm{P})$ and the resultant flow rate $(\mathrm{q})$. 


\section{K. Model comparisons}

9.10 In the interest of quantifying the QFFM relative to other conventional flow models, we include a comparison to the Poiseuille [46] and Blasius [47] models in the QFFM frame of reference, shown below in Fig. 16. The Poiseuille model pertains to the laminar region and the Blasius model is supposed to be accurate over some specified narrow region of the turbulent regime.

\section{Fig. 16}

\section{Comparison of QFFM , Blasius and Poiseuille}

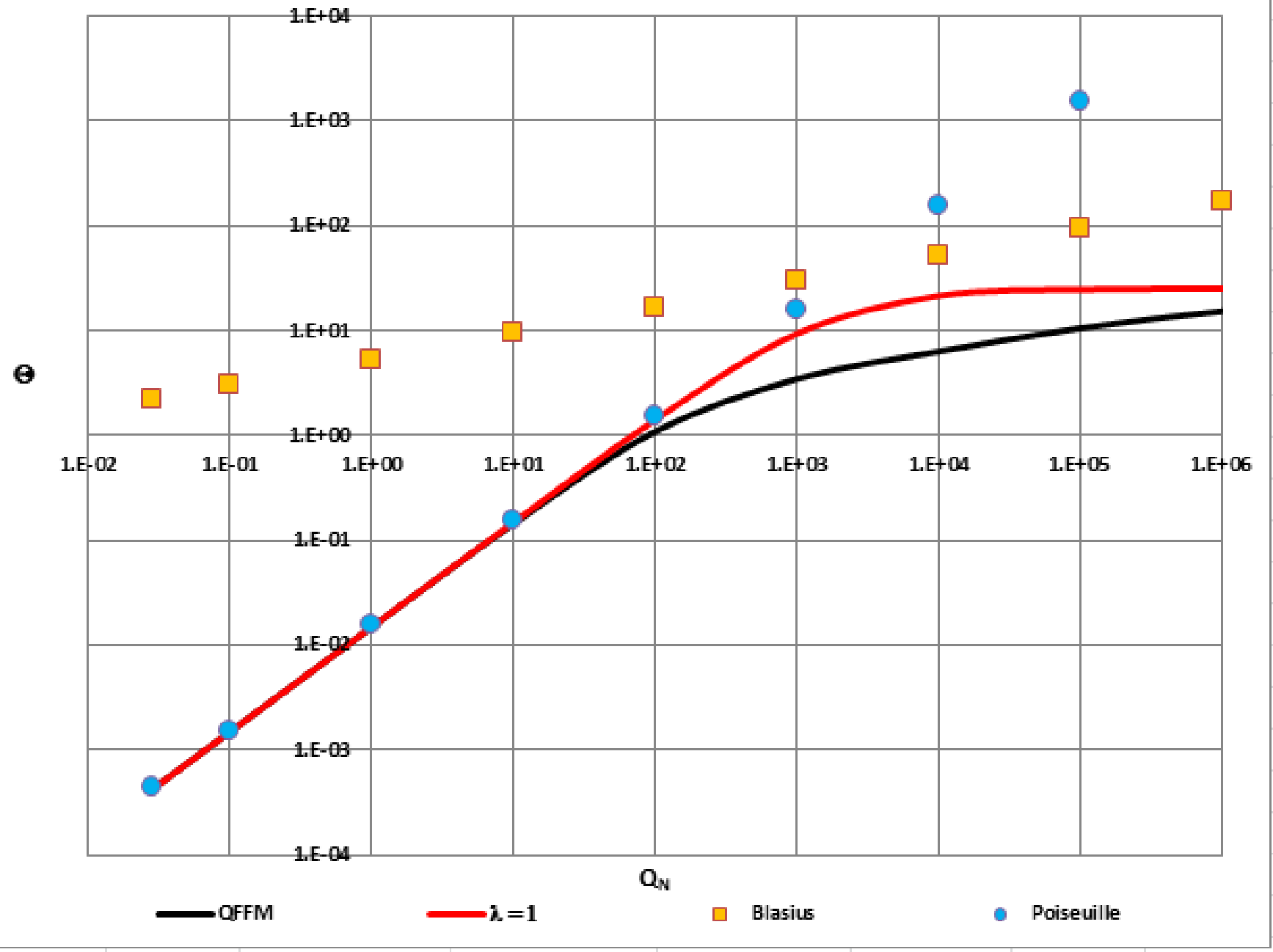

As shown in Fig. 16, the QFFM and the Poiseuille models are very closely matched at low values of $\mathrm{Q}_{\mathrm{N}}$, the region in which the Poiseuille model is generally regarded as being valid. The Blasius model, on the other hand, completely misses the mark across the entire region of the flow regime. It is off by an order of magnitude in the wrong direction at its closest point to the QFFM, which, curiously, falls in the transitional region of the flow regime, i.e., the region of the flow regime corresponding to the so-called "Nikuradze inflection profile". Accordingly, we suggest that the Blasius model is virtually worthless. 


\section{Conclusions}

As of this writing, the Navier-Stokes equation for fluid flow stands without analytical solution. We do not say if this is because the currently accepted/popular theories of fluid flow in closed conduits are fatally flawed, or because the equation itself is necessarily deficient.

In this paper, we have derived a unique theory of fluid flow in closed conduits. We do not claim that our theory provides a "solution" to the Navier-Stokes equation per se, but we feel confident that it correctly and uniquely describes fluid flow in closed conduits.

Probably the most important disclosure herein, relative to conventional wisdom, is that the conventional Reynolds number, by itself, is inadequate to define kinetic contributions to overall conduit permeability. In its place, we have defined the "fluid current" as the governing kinetic parameter which incorporates all contributing elements to the fluid flow regime.

In addition, and with almost equal implications, the concept of "chaotic flow profile" has been shown herein to be unfounded. Instead, we have provided a predictable and structurally stable, pattern of fluid flow profile, which is directly related to the fluid flow embodiment and instantaneous operating conditions in any given experiment under study.

Finally, this paper puts to rest, once and for all, the myth that packed and empty conduits belong to two different flow regimes. On the contrary, it is now clear that both are governed by the exact same Laws of Nature.

\section{Acknowledgement}

I wish to acknowledge Hubert M Quinn Jr., my son, for his engineering contribution in designing a continuous fluid flow loop, which enables visualization of many of the underlying concepts described in this paper.

\section{References}

[1] H. Darcy, Les Fontaines Publiques de la Ville de Dijon, Victor Dalmont, Paris, France, 1856

[2] E. Erdim, O. Akgiray, I. Demir; A revisit of pressure drop-flow rate correlations for packed beds of spheres, Powder Technology 283 (2015) 488-504,

[3 ] N. Dukhan, O. Bagci, M. Ozdemir. Experimental flow in various porous media and reconciliation of Forchheimer and Ergun relations. Experimental Thermal and Fluid Science 57 (2014) 425-433.

[4] CHARLES L. FEFFERMAN, EXISTENCE AND SMOOTHNESS OF THE NAVIER-STOKES EQUATION

http://www.claymath.org/sites/default/files/navierstokes.pdf

[5] H.M. Quinn, J. J. Takarewski, E. Williams, US 7,767,463, Method for screening mobile phases in chromatography systems (2010)

[6] H. M. Quinn, J. E. Brann, III, US 6,149,816, Chemical analyses (2000)

[7] H. M. Quinn, John E. Brann, III, US 6,110,362, Chemical analyses (2000)

[8] H. M. Quinn, R. A. Menapace, C J. Oberhauser, US 5,968,367, High performance liquid chromatography method and apparatus (1999)

[9] H. M. Quinn, J.J Takarewski, US 5,919,368, High performance liquid chromatography method and apparatus (1999)

[10] H. M. Quinn, R. A. Menapace, C J. Oberhauser US 5,795,469, High performance liquid chromatography method and apparatus (1998) 
[11] H. M. Quinn, J. J. Takarewski, US 5,772,874, High performance liquid chromatography method and apparatus (1998)

[12] H. M. Quinn, US 20100071444, Throughput Screening, Purification and Recovery System for Large and Small Molecules (2010)

[13] G. Guiochon, S. G. Shirazi, and A. M. Katti, Fundamentals of Preparative and Nonlinear Chromatography, Academic Press, Boston, Mass, USA, 1994.

[14] R. Endele, I. Halasz, and K. Unger, J. Chromatography, 99, 377 (1974).

[15] D. Cabooter, J. Billen, H. Terryn, F. Lynen, P. Sandra, G. Desmet; Journal of Chromatography A, 1178 (2008) 108-117

[16] F. Gritti, D. S. Bell, G. Guiochon; Journal of Chromatography A, 1355 (2014) 179-192,

[17] Kim Vanderlindena, Gert Desmeta, David S. Bellb, Ken Broeckhoven, Detailed efficiency analysis of columns with a different packing quality and confirmation via total pore blocking, Journal of Chromatography A, 1581-1582 (2018) 55-62

[18] U. Neue, HPLC Columns-Theory, Technology and Practice, Wiley-VCH, 1997.

[19] A. E. Reising, J. M. Godinho, K. Hormann, J. W. Jorgenson, U. Tallarek. Larger voids in mechanically stable, loose packings of $1.3 \mathrm{~mm}$ frictional, cohesive particles: Their reconstruction, statistical analysis, and impact on separation efficiency: Journal of Chromatography A, 1436 (2016) 118-132.

[20 ] P. C. Carman, "Fluid flow through granular beds," Transactions of the Institution of Chemical Engineers, vol. 15, pp. 155-166, 1937

[21] R. B. Bird, W. E. Stewart, and E. N. Lightfoot, Transport Phenomena, John Wiley \& Sons, 2002.

[22 Dejan Brki'c and Pavel Praks, Unified Friction Formulation from Laminar to Fully Rough Turbulent Flow; Appl. Sci. 2018, 8, 2036; doi:10.3390/app8112036

[23] M. Rhodes, Introduction to Particle Technology, John Wiley \& Sons, 1998

[24] L. Prandtl, in Verhandlungen des dritten internationalen Mathematiker-Kongresses in Heidelberg 1904, A. Krazer, ed., Teubner, Leipzig, Germany (1905), p. 484. English trans. in Early Developments of Modern Aerodynamics, J. A. K. Ackroyd, B. P. Axcell, A. I. Ruban, eds., Butterworth-Heinemann, Oxford, UK (2001), p. 77.

[25] K.E. Bullen, An Introduction to the Theory of Mechanics, $7^{\text {th }}$ edition, Cambridge AT THE UNIVERSITY PRESS 1965.

[26] J. C. Giddings, Dynamics of Chromatography, Part I: Principles and Theory, Marcel Dekker, New York, NY, USA, 1965.

[27] J. C. Giddings, Unified Separation Science, John Wiley \& Sons, 1991

[28] K. B. SOUTHERLAND, R. D. FREDERIKSEN , W. J. A. DAHM; Comparisons of Mixing in Chaotic and Turbulent Flows; Chaos, Solitons \& Fractals Vol. 4, No. 6, pp. 1057-1089, 199

[29] T. Farkas, G. Zhong, G. Guiochon, Validity of Darcy's Law at Low_Flow Rates in Liquid Chromatography Journal of Chromatography A, 849, (1999) 35-43

[30] H.M. Quinn; A Reconciliation of Packed Column Permeability Data: Column Permeability as a Function of Particle Porosity Journal of Materials Volume 2014 (2014), Article ID 636507, 22 pages http://dx.doi.org/10.1155/2014/636507

[31] J. M. Coulson; University of London, Ph.D. thesis, "The Streamline Flow of Liquids through beds comprised of Spherical particles" 1935.

[32] Ergun, S. and Orning, A.A., Fluid Flow through Randomly Packed Columns and Fluidized Beds, Ind. Eng. Chem. vol. 41, pp. 1179, 1949.

[33] Ergun, S., Determination of Particle Density of Crushed Porous Solids, Anal. Chem. vol. 23, 1951

[34] Ergun, S., Fluid Flow Through Packed Columns, Chem. Eng. Progr. vol. 48, pp. 89-94, 1952

[35] H.M. Quinn, A Reconciliation of Packed Column Permeability Data: Deconvoluting the Ergun Papers Journal of Materials Volume 2014 (2014), Article ID 548482, 24 pages http://dx.doi.org/10.1155/2014/548482

[36] H. M. Quinn, Reconciliation of packed column permeability data-part 1: the teaching of Giddings revisited, Special Topics \& Reviews in Porous Media, vol. 1, no. 1, pp. 79-86, 2010. 
[37] B.J. Mckeon, C.J. Swanson, M.V. Zagarola, R.J. Donnelly and A. J. Smits. Friction factors for smooth pipe flow; J.Fluid Mech. (2004), vol. 511, pp.41-44. Cambridge University Press; DO1;10.1017/S0022112004009796.

[38] B.J. Mckeon, M.V. Zagarola, and A. J. Smits. A new friction factor relationship for fully developed pipe flow; J.Fluid Mech. (2005), vol. 238, pp.429-443. Cambridge University Press; DO1;10.1017/\$0022112005005501.

[39] C.J. Swanson, B. Julian, G. G. Ihas, and R. J. Donnelly. Pipe flow measurements over a wide range of Reynolds numbers using liquid helium and various gases. J. Fluid mech. (2002), vol. 461, pp.51-60. Cambridge University Press; DO1;10.1017/S0022112002008595.

[40] J. Nikuradze, NASA TT F-10, 359, Laws of Turbulent Flow in Smooth Pipes. Translated from "Gesetzmassigkeiten der turbulenten Stromung in glatten Rohren" VDI (Verein Deutsher Ingenieure)-Forschungsheft 356.

[41] H. M. Quinn, Unpublished data.

[42] j. Nikuradze, NACA TM 1292, Laws of Flow in Rough Pipes, July/August 1933. Translation of “Stromungsgesetze in rauhen Rohren." VDIForschungsheft 361. Beilage zu " Forschung auf dem Gebiete des Ingenieurwesens" Ausgabe B Band 4, July/August 1933.

[43] M. DOBRNJAC, DETERMINATION OF FRICTION COEFFICIENT IN TRANSITION FLOW REGION FOR WATERWORKS AND PIPELINES CALCULATION, MECHANICAL ENGINEERING FACULTY, UNIVERSITY IN BANJALUKA, BANJALUKA, REPUBLIC SRPSKA, BOSNIA \& HERZEGOVINA

[44] A. Ramakrishna Rao, B. Kumar, Friction Factor for Turbulent Pipe Flow, Department of Civil Engineering, IISc, Bangalore-560012, India

[45] B. H. Yang, D. D. Joseph, Virtual Nikuradse, Journal of Turbulence Vol. 10, No. 11, 2009, 1-28

[46] J.L.M. Poiseuille, Memoires des Savants Etrangers, Vol. IX pp. 435-544, (1846); BRILLOUIN, M. (1930) Jean Leonard Marie Poiseuille. Journal of Rheology, 1, 345.

[47] H. Blasius (1908). "Grenzschichten in Flüssigkeiten mit kleiner Reibung". Z. Angew. Math. Phys. 56: 1-37 